\title{
STRAIN AND INFLATION-UNEMPLOYMENT RELATIONSHIP IN TRANSITIONAL ECONOMIES: A THEORETICAL AND EMPIRICAL INVESTIGATION*
}

\author{
Lucian Liviu Albu**
}

1997

\begin{abstract}
Standard economic theory tells that a command system, like the former eastern economies, allocates resources poorly due to the impossibility of accurate calculation. Therefore, once prices are freed and start to operate at quasi-equilibrium (market-clearing) levels, the hidden inefficiencies come into the open and a possible massive resource reallocation would have to take place. More precisely, the issue refers to the possible and probable intensity of resource reallocation in view of constraints like the balance between exit and entry in the labour market, the size of the budget deficit and the means for its non-inflationary financing, social and political stability, etc. This paper tries to conceptualise the fact that the dynamics of unemployment and inflation are correlated not in a classical sense but in a very complicated mode that suggests the occurrence of some attractors when certain slow parameters are evolving in the neighbourhood of special threshold-values. The start is made with simple models that are based on empirical data and can show to us the traces to discover the steps of transition in eastern economies on the inflation-unemployment relationship space. Then, using economic theory combined with non-linear modelling more refined information is extracted from the statistical standardised data for to evaluate other faces of the eastern transition.
\end{abstract}

Keywords: natural rate of unemployment, potential function, Hurst exponent, pitchfork bifurcation, modified Phillips curve, Rössler attractor

JEL classification: C61, C65, E24, E27, E32

* This research was undertaken with support from the European Commission's Phare ACE Programme 1996, Contract Number: P96-6657-F.

** Institute for Economic Forecasting, Romanian Academy, 13, Calea 13 Septembrie, Casa Academiei, Sector 5, Bucharest, Romania; e-mail: liviul@hotmail.com; albu_lucian_liviu@yahoo.com. 


\author{
UNIVERSITY OF LEICESTER \\ DEPARTMENT OF ECONOMICS \\ CENTRE FOR EUROPEAN ECONOMIC STUDIES
}

\title{
STRAIN AND INFLATION-UNEMPLOYMENT RELATIONSHIP IN TRANSITIONAL ECONOMIES: A THEORETICAL AND EMPIRICAL INVESTIGATION*
}

\author{
Lucian Liviu ALBU** \\ (Academic Visitor within ACE-PHARE Programme)
}

LEICESTER

December 1997

* This research was undertaken with support from the European Commission's Phare ACE Programme 1996, Contract Number: P96-6657-F.

** Institute for Economic Forecasting, Romanian Academy, 13, Calea 13 Septembrie, Casa Academiei, Sector 5, 76117, Bucharest, Romania, Tel: 40-1-4103200/3319: Mailing address: 6, Costache Stamate, BI. 3E, Ap. 25, Sector 4, 751894, Bucharest; Tel: 40-1-3306587; Fax: 40-13306587; E-mail: lucianl@sunu.rnc.ro; lla2@le.ac.uk; liviul@hotmail.com; llalbu@valhalla.racai.ro; aalbu@pcnet.pcnet.ro; livl@usa.net. 


\section{Acknowledgments}

The author gratefully acknowledges the financial support of ACE-Phare Programme for a six-months stay at Leicester University. In the same time, he would like to thank Professor Wojciech Charemza for his invaluable support and encouragement, without this work could not have been written, and for the favourable conditions to work at Economics Department. 


\section{- Abstract -}

Standard economic theory tells that a command system, like the former eastern economies, allocates resources poorly due to the impossibility of accurate calculation. Therefore, once prices are freed and start to operate at quasiequilibrium (market-clearing) levels, the hidden inefficiencies come into the open and a possible massive resource reallocation would have to take place. More precisely, the issue refers to the possible and probable intensity of resource reallocation in view of constraints like the balance between exit and entry in the labour market, the size of the budget deficit and the means for its non-inflationary financing, social and political stability, etc.

This paper tries to conceptualise the fact that the dynamics of unemployment and inflation are correlated not in a classical sense but in a very complicated mode that suggests the occurrence of some attractors when certain slow parameters are evolving in the neighbourhood of special threshold-values. The start is made with simple models that are based on empirical data and can show to us the traces to discover the steps of transition in eastern economies on the inflation-unemployment relationship space. Then, using economic theory combined with non-linear modelling more refined information is extracted from the statistical standardised data for to evaluate other faces of the eastern transition. 


\section{Introduction}

The actual transition in eastern countries looks like a provocation for both economic theory and quantitative evaluations. Continuing to be poor the data on the transitional economies are increasing. That is more important is the fact that in an important measure they are remaining under a large degree of incoherence in the usual sense in economic literature. However, important steps have been made to model by econometric methods. At this point it is necessary to remark the advanced econometric studies on the transition in eastern countries achieved by a specialised group within the University of Leicester under the co-ordination of Wojciech Charemza. In this sense, a research on the transition coming from other point of view such as non-linear modelling would be as a modest additional step. Also, in economic research last years there is a trend to approach the same economic problem or phenomenon coming from the two apparently opposite directions.

One of the most important goals of the paper is to demonstrate that the actual transition in eastern countries is going to be integrated within a more general process of a long-run economic transition that also includes western countries. The main problem for eastern transition is if they must follow the same stages as western countries in the past or there are some possibilities to avoid some of them.

More implicitly, the transition is accompanied by an increasing strain in the economic system and surely the inflation-unemployment relationship has the leader role in this equation. In this idea, to research the conditions in which the speed of transition may be increased or to search that of occurrence of attractors is one of the best ways to describe the strain of the system.

The paper is organised in five sections. First focuses on the relevance of the advancing process in the transition way, based mainly on the statistical data support. Some rules to evaluate the speed of transition or the stage where it is placed in eastern countries are extracted by using several simple models. Also, a simple model derived from the potential-function technique is used to find if the inscription of eastern economies on a relative stable long-run way has been achieved. Second section concentrates on the possibility to apply some results of the so-called bifurcation theory for detecting the main features manifested in the long-run evolution of the unemployment and inflation respectively. A schedule of a methodology to detect the attracting zones in the inflation-unemploymentproduction space is presented, including an application on a western country. More comparisons between countries are presented in case of a truncated bifurcation model. The last two sections deal with a promising model that demonstrates the possibility of emergence of a chaotic motion in three-dimensional systems and respectively with a discrete-time model of the modified Phillips curve. In section four is presented a model that can generate a spiral-type chaos in case of the inflationunemployment cycle. On this base we hope to capture, in case of applications, some important features of the type of transition. Section five includes several simulation results coming from the change of so-called state parameters in case of a discrete model including errors in expected inflation and a correcting factor in sense of adaptive expectations. 


\section{Intuitive transitions}

\subsection{Some explanations of new trends}

Empirical data demonstrate, on the background of business cycles, some major changes of trends in Western countries in the last 25 years. Among these we can mention an impressive decrease in inflation followed by a continuing growth of unemployment and general diminution of the growth rate of production (GDP).

An important question is if a smaller area of main macroeconomic indicators' variation represents greater economic stability and, relatively, less strain.

In any case the evolution is from a period where the main symptom of strain was inflation toward one where unemployment became the principal symptom and factor of strain. On the other hand, this evolution means that on the unemploymentside occurred a relaxation, higher levels of unemployment being viewed as normal but not the case for the inflation level.

A deeper analysis shows the possibility of some persistent trends and longrun attractors. To demonstrate this assertion we present in Annex 1a the graphical representation of unemployment, inflation, and respectively inflation-unemployment, for the period 1970-1996, with one-year lag, in case of three western countries.

On the other hand, in Eastern European countries there was an opposite situation after 1989; open inflation rose rapidly in the region whereas unemployment did also rise but at a smaller pace. Probably, the long-term trends will be similar to that registered in Western countries. The most important question is how long this transition period will be. In such context to estimate strain and to evaluate the costs of its diminution becomes an important topic for research. As example of the nonstructured time-series, less that of unemployment, in case of eastern countries, in Annex $1 \mathrm{a}$ is also presented the monthly evolution of the same indicators in case of Romania for about 50 months in the period 1990-1996.

To illustrate the occurrence of some limit cycles in case of western countries and the non-clear situation (taking into account the short period of reference) in eastern countries we plotted in polar coordinates the dynamics of unemployment and respectively of inflation in Annex 1b.

\subsection{Two simple models of transition}

In this section we present two models that describe some possible ways from the actual situation of eastern economies to one minimal requested for the acceptance within the UE.

First model assumes a simple linear correlation between the registered rate of unemployment (x) and the yearly rate of the GDP change (vy). Also, the inflation rate by year $(z)$ is expressed as a parabolic function related to the same yearly rate of GDP modification. The model is described by the following equations:

$$
\begin{aligned}
& x(v y)=a \cdot v y+x n \\
& z(v y)=A \cdot v y^{2}+C
\end{aligned}
$$


where a, A, C are constants, and $x$ is the "natural" level of unemployment. Also, replacing $v y$ in the inflation function by separating it from the equation (2.1) we can write:

$z(x)=A \cdot[(x-x n) / a]^{2}+C$

The results of applying the model on the disposable data for three western countries (Belgium, France, and UK) and for five eastern countries (Bulgaria, Czech Republic, Hungry, Poland, and Romania) are presented in Table 1. In case of western countries the period is 1970-1996 and in that of eastern countries is 19901996.

Table 1

\begin{tabular}{llll}
\hline $\mathrm{aB}:=-0.996$ & $\mathrm{xnB}:=0.114$ & $\mathrm{AB}:=2.468$ & $\mathrm{CB}:=0.048$ \\
$\mathrm{aF}:=-1.027$ & $\mathrm{xnF}:=0.102$ & $\mathrm{AF}:=-3.024$ & $\mathrm{CF}:=0.065$ \\
$\mathrm{aUK}:=0.068$ & $\mathrm{xnUK}:=0.074$ & AUK $:=-7.198$ & $\mathrm{CUK}:=0.093$ \\
$\mathrm{aBULG}:=0.299$ & $\mathrm{xnBULG}:=0.128$ & ABULG $:=156.982$ & $\mathrm{CBULG}:=0.376$ \\
$\mathrm{aCZ}:=-0.056$ & $\mathrm{xnCZ}:=0.027$ & ACZ $:=22.073$ & $\mathrm{CCZ}:=0.094$ \\
$\mathrm{aHUN}:=0.304$ & $\mathrm{xnHUN}:=0.099$ & AHUN $:=7.891$ & $\mathrm{CHUN}:=0.238$ \\
$\mathrm{aPOL}:=0.47$ & $\mathrm{xnPOL}:=0.131$ & APOL $:=449.77$ & $\mathrm{CPOL}:=-0.776$ \\
$\mathrm{aR}:=0.352$ & $\mathrm{xnR}:=0.08$ & AR $:=18.567$ & $\mathrm{CR}:=1.253$
\end{tabular}

Despite the short period in case of the eastern countries it can be extracted some ideas from the table. So, it seems a convergence relating the natural rate of unemployment, some of the eastern countries (especially, Czech Republic) having even a smaller calculated natural rate of unemployment then western countries. The remaining problem of the transition in these countries is the high rate of inflation. The model was conceived supposing the so-called stagflation phenomenon, i.e., the simultaneous presence of inflation and zero growth rates of the GDP, coupled with nonzero and usually high unemployment rates. In literature, as attempt to model this phenomenon consists of modifying the original Phillips curve by introducing additional influences like, e.g., the expected inflation rate $\pi^{\mathrm{e}}$ [1]. In a sense, in the context of the model, the parameter $C$ can be viewed as the "expected" inflation rate. In Figure 1 it is shown the function of inflation in considered countries, from where the higher strain in case of the eastern group is evident. To extract the way of transition we present in parallel the inflation and the unemployment functions in two groups of countries in Figure 2. Without the differences between levels, the discrepancy is now clear: in western countries the line of unemployment intersects the inflation parabolas but in eastern countries this is not happened till the present. A possible way, so-called the transition in three stages, should be firstly to move down the inflation level simultaneous with an increasing in unemployment level (requested also by the economic restructuring in eastern group) till their intersection and then, in the future, to change the type of inflation parabola (like is the case in western group, from the situation of Belgium to that registered in France and UK).

The second model represents a development of the first model by reformulating the unemployment function, as following: 
$x(v y)=a \cdot v y^{2}+b \cdot v y+c$

where $\mathrm{b}$ is also a coefficient. The computing data, resulted by applying the model, are presented synthetically in Table 2 .
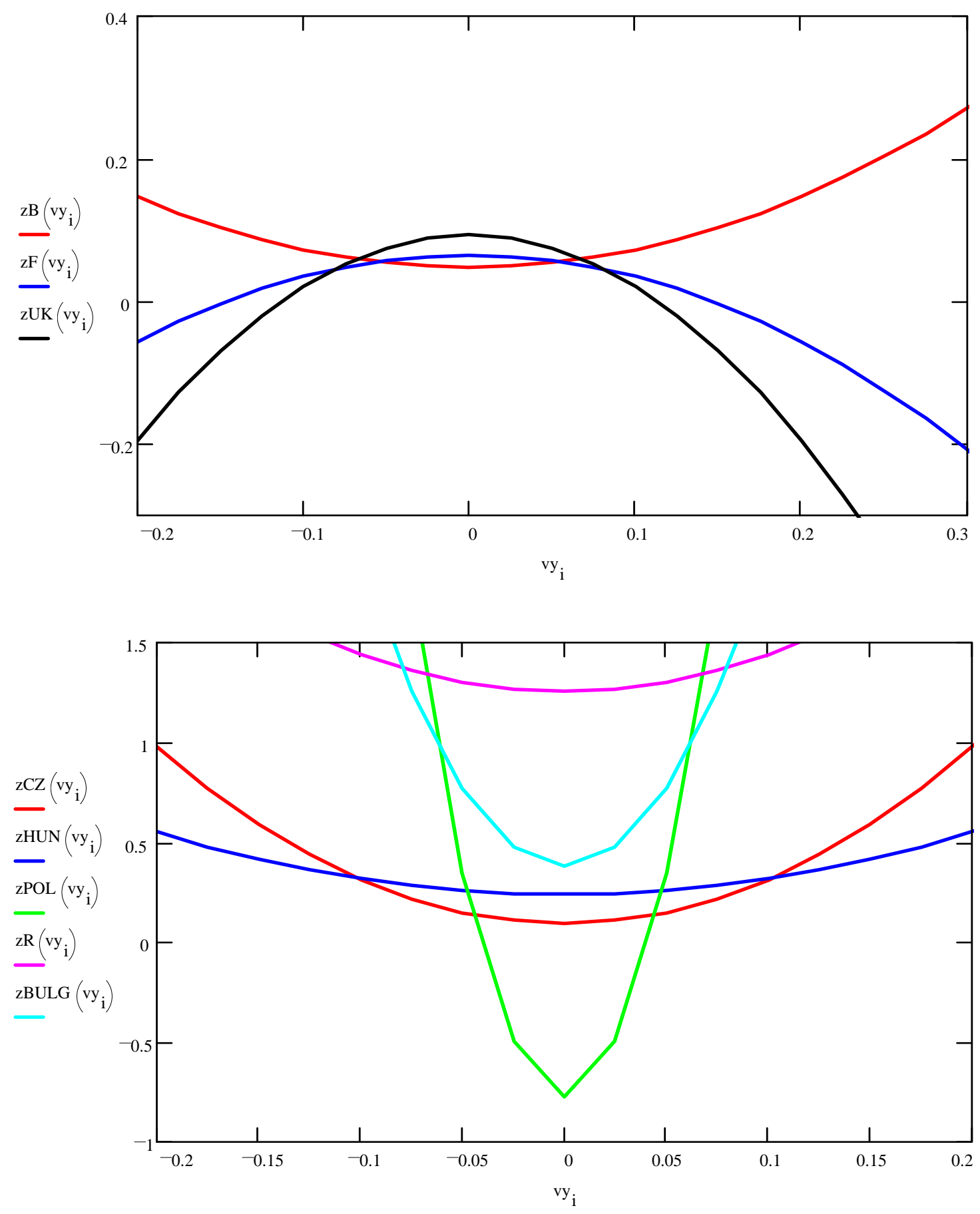

Figure 1 

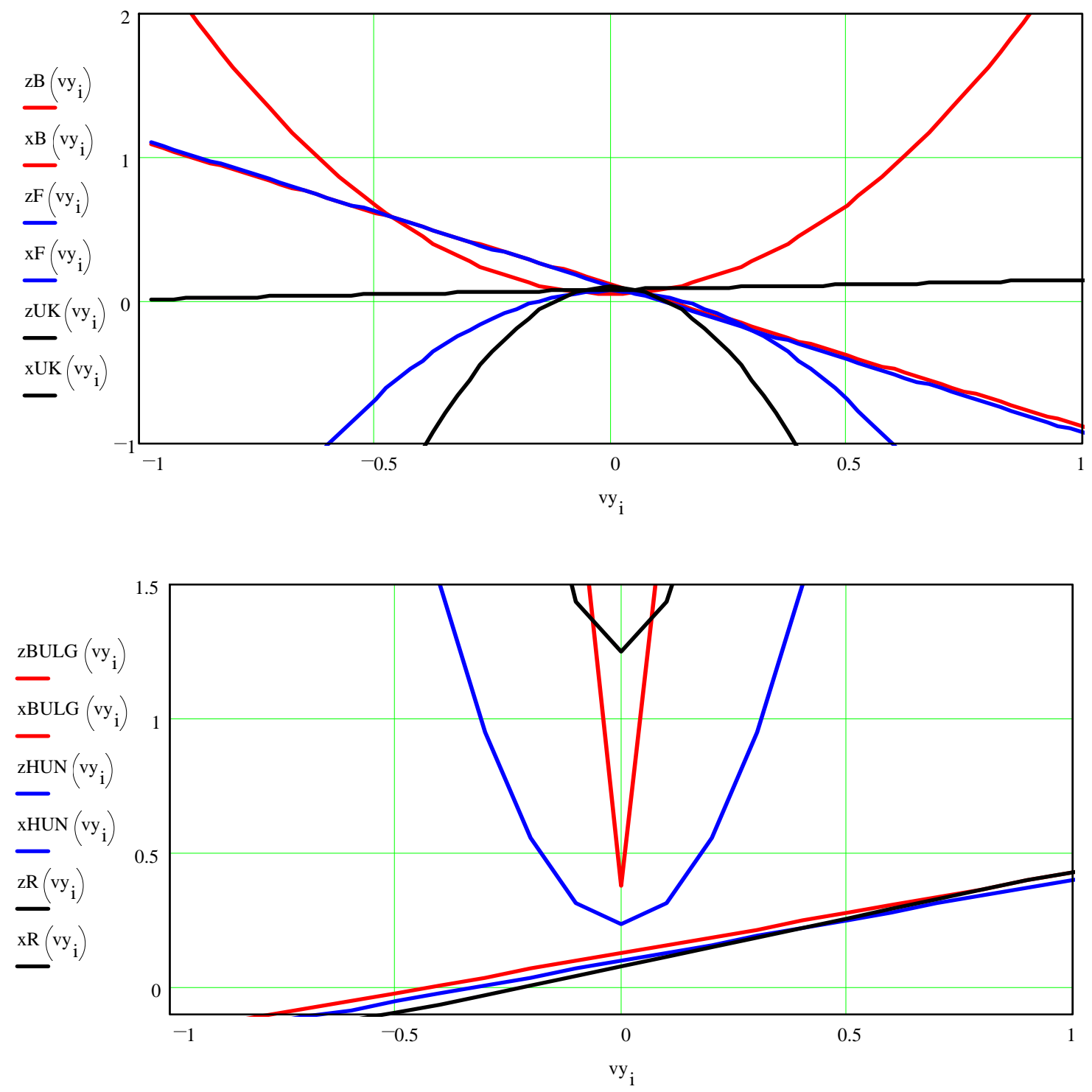

Figure 2

Table 2

\begin{tabular}{|c|c|c|c|c|}
\hline $\mathrm{aB}:=-29.989$ & $\mathrm{bB}:=0.451$ & $=0.11$ & $:=2.468$ & $=0.048$ \\
\hline$:=-35.51$ & $\mathrm{bF} \quad:=0.758$ & $=0.089$ & $\mathrm{AF}:=-3.024$ & $=0.065$ \\
\hline aUK $:=-9.936$ & bUK $\quad:=0.532$ & $:=0.073$ & AUK $:=-7.198$ & $=0.093$ \\
\hline aBULG $:=-6.178$ & bBULG $:=-0.235$ & cBULG $:=0.135$ & ABULG := 156.982 & CBULG $:=0.376$ \\
\hline $\mathrm{aCZ}:=1.507$ & $:=0.093$ & $:=0.024$ & $\mathrm{ACZ}:=22.073$ & $:=0.094$ \\
\hline aHUN $:=3.596$ & bHUN $:=0.645$ & $:=0.097$ & AHUN $:=7.891$ & $=0.238$ \\
\hline aPOL :=- 3.899 & $\mathrm{bPOL} \quad:=0.281$ & сPOL & APOL $:=449.77$ & $\mathrm{CPOL}:=-0.776$ \\
\hline$:=-0.055$ & $:=0.349$ & $=0.08$ & $=18.567$ & $=1.253$ \\
\hline
\end{tabular}




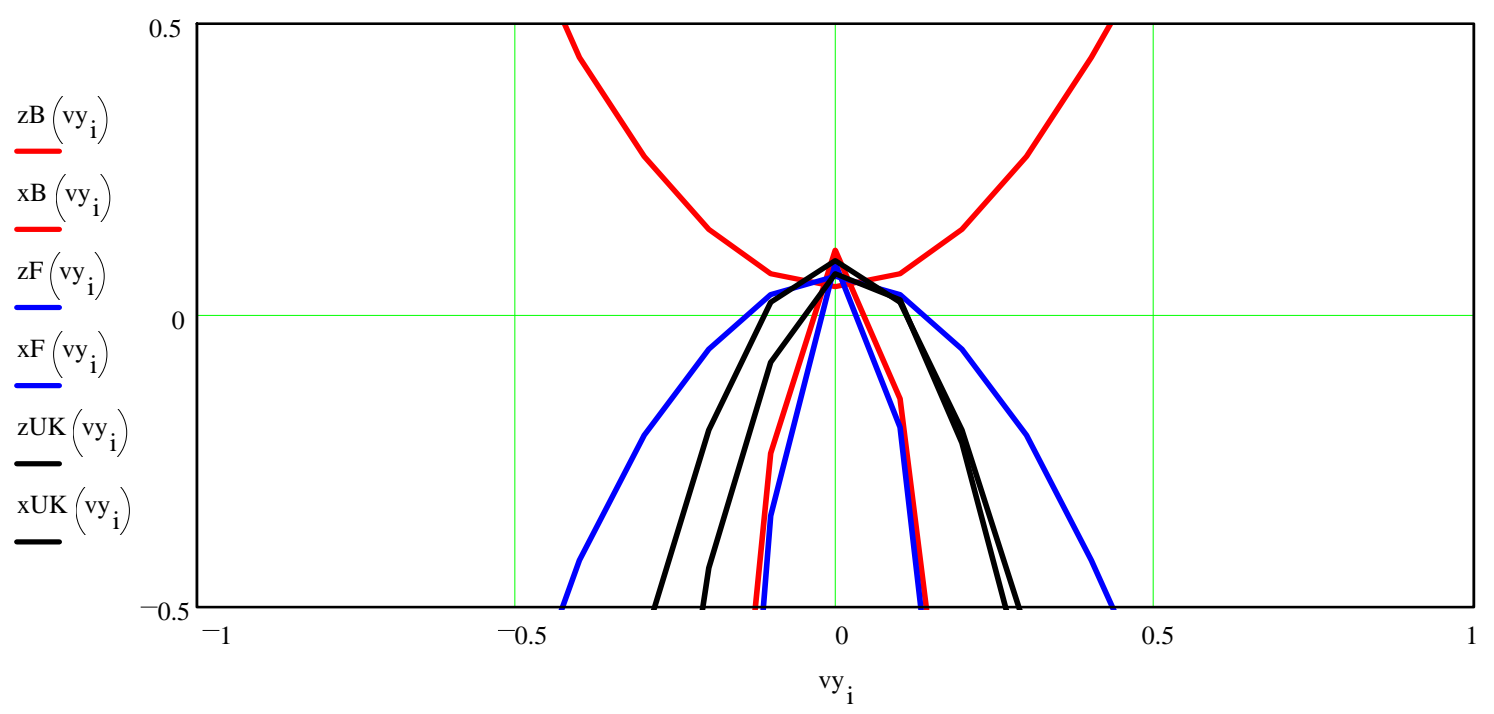

Figure 3
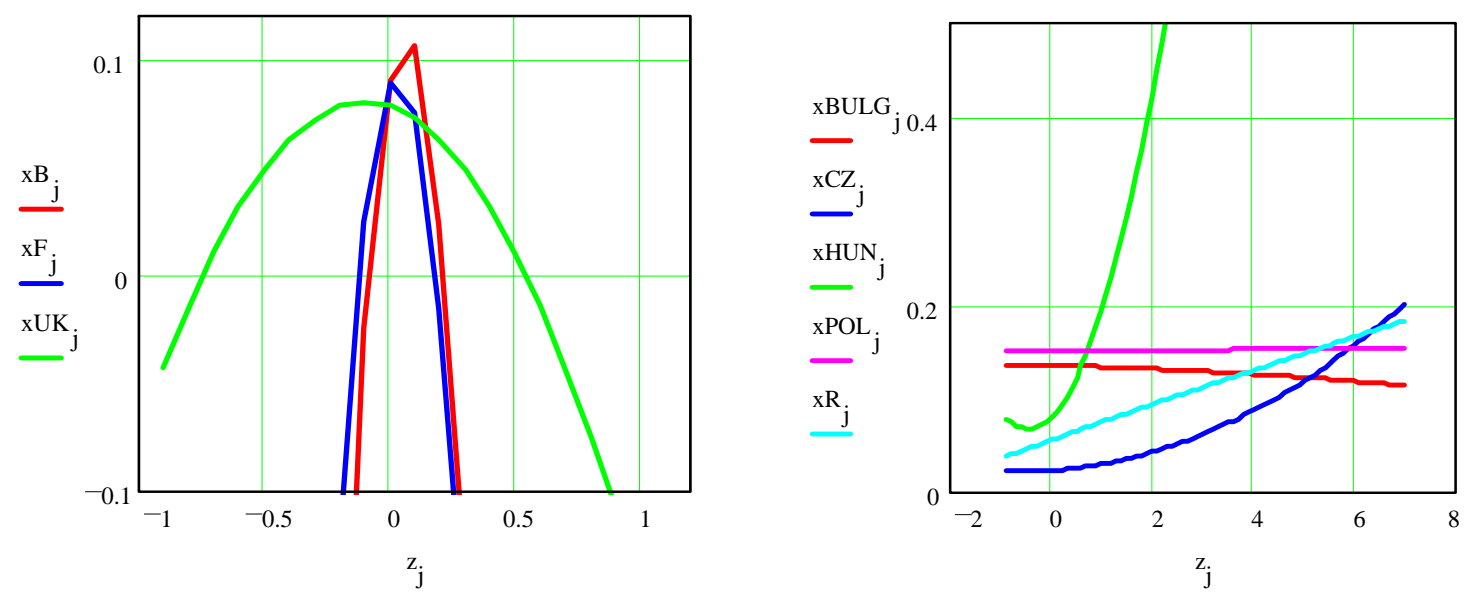

Figure 4

Again there are fundamental differences between the two groups of countries: since in western countries the two curves ( $x$ and $z$ ) are placed in a closed region of the map, in eastern countries the aria of distribution is large, existing no point of intersection. In this sense, we present in Figure 3 the map having as abscise the yearly changing rate of GDP and in Figure 4 the map of unemployment rate function relating to that of the yearly inflation level.

Coming from this model we should imagine five steps of the transition:

1 - a severe decreasing of GDP, followed by an important growth of inflation, but a modest increasing of unemployment (the opposition of the former structure and the trust that the transition can be passed with a high level of occupation of labour force);

2 - an explosion in the inflation level, followed by a smaller rate of GDP decreasing, and a severe increasing of unemployment till the genuine restructuring of the economy is achieved;

3 - after the maximum level of unemployment (requested by restructuring), 
and the stop of GDP decreasing it follows a re-improvement of production, a reabsorption of an important part of unemployment (but on the base of the new economic structure), and a temporisation of inflation that although is situated at one high level;

4 - the rate of increasing GDP rich the maximum level and then it come down with a small diminution of unemployment (that is near the minimum level), and, most important, an accelerate decreasing of inflation till the level registered in western countries 25 years ago (this stage corresponding to the stage of western economies at the end of 60's);

5 - the beginning of one new increasing of unemployment but in condition of a small and controlled level of inflation.

\subsection{Inflation-unemployment relationship derived from a potential function}

Coming from some old papers (Albu, 1995; Daianu and Albu, 1996) we apply a simple model that can give us a measure of the stability-degree of inflationunemployment relationship in European economies.

Let $S$ being the sum $S$ of unemployment and inflation:

$S=u+p$

where $u$ is unemployment rate and $p$ is the inflation rate, and let $P$ their product:

$P=u \cdot p$

We write the shares of $u$ and respectively $p$ in $S$ by $x 1$ and respectively $x 2$ as following:

$\mathrm{x} 1=\mathrm{u} / \mathrm{S}$ and $\mathrm{x} 2=\mathrm{p} / \mathrm{S}$

and their product as:

$P P=(u / S) \cdot(p / S)$

Now, let consider the generalised variable $x$ representing shares of $S$ such as the model can be expressed by the following system of equations:

$p(x)=x \cdot S$

$u(x)=S-p(x)$

From this it results the following expression of PP:

$P P(x)=x \cdot(1-x)$

In order to investigate the behaviour of the system in time, let consider that the main equation of our model can be derived from an existing potential function $\mathrm{V}$ $(\mathrm{x} ; \mathrm{m})$ :

$\mathrm{dV} / \mathrm{dx}=0$

where $\mathrm{x}$ is the rapid variable of the system and $\mathrm{m}$ - the slow or control variable (both 
are, implicitly, functions of time). In our case, we chose the following potential function:

$V(x ; m)=\left(-x^{3}\right) / 3+\left(x^{2}\right) / 2-m \cdot x$

to which it corresponds the following equation of potential surface:

$-x^{2}+x-m=0$

Comparing this with the relation of PP already obtained it results that in terms of our model the slow variable $\mathrm{m}$ can be estimated by the following expression:

$\mathrm{m}=\mathrm{P} /\left(\mathrm{S}^{2}\right)$

Considering the analysis of the graph of function $\mathrm{V}$, it results some threshold values of the parameter $\mathrm{m}$. Therefore, for $\mathrm{x}$ having values among 0 and 1 , there are the following cases:

when $0<m<3 / 16, V$ has 3 real roots ( 0 and other two separated);

when $\quad m=3 / 16, V$ has 2 real roots ( 0 and an other double root);

when $3 / 16<m<1, V$ has only 0 as real root .

The graph of function $\mathrm{V}$ is shown in Figure 5.

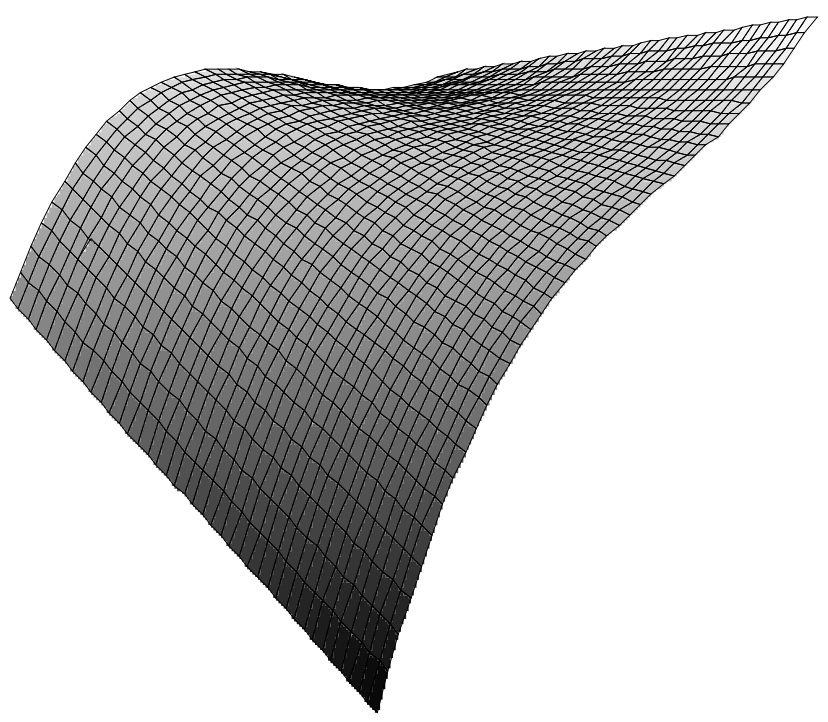

$\mathrm{M}$

Rotation 50 (0 to 360) Tilt 10 (0 to 360 )

Vertical Scale 100 (1 to 100 magnifications)

Figure 5

A very important threshold value of parameter $m$ is $1 / 4$, where the maximum, minimum, and inflexion points are confounded. Other important conclusions concerning evolution and stability of the system are: there are two equilibrium curves on the potential surface - a stable equilibrium curve $(\mathrm{C} 1)$ and an unstable equilibrium curve (C2); for values of $x$ smaller than $(\mathrm{C} 2)$ the trajectories are 
attracted to $(\mathrm{C} 1)$ (the long-run effect); for value of $x$ greater than $(\mathrm{C} 2)$ the system is strong attracted in an intense troubled zone; a rich menu of alternatives can be deduced by investigation of the function $\mathrm{V}$ map moreover this can offer some larger possibilities of statistical data interpretation.

A decisive problem represents the estimation of $m$. If this were quantified we would adopt desired alternatives by knowing their consequences. The resulting decisive importance of quantifying $m$ strongly contradicts the authors who consider unemployment-inflation relationship having insignificant or neutral influence on the entire economic system. Consequently it must be ignored or both its factors must be strongly repressed simultaneously. When $\mathrm{m}$ equals $1 / 4$ the system lost all equilibrium branches and $u=p$. However, the remaining question is how can one solve better the problem of stability within zone $(0 ; 0.25)$. For this we present a short version of a discrete-time model as an alternative.

Considering parameter $\mathbf{a}$ as an essential parameter that assures the dynamics of the system in case of the discrete model, the equation will be:

$x_{t}=a \cdot x_{t-1} \cdot\left(1-x_{t-1}\right)$

This equation (which represents a canonical form of May's equation) possesses a wide range of dynamic behaviour, which is well known in the specialised literature (May, 1976). Limiting our attention to the initial conditions of the dynamics of $x$ included in the interval [ $0 ; 1$ ], the following "windows" of a were identified: $0<a<1, x$ moves monotonously towards the stationary solution $x=0 ; 1$ $<a<2, x$ moves monotonously towards the stationary solution $x=(a-1) / a ; 2<a$ $<3$, $x$ converges with a flattened oscillatory movement to the stationary solution $x=$ (a-1) / $a ; 3<a<4$, $x$ demonstrates a complex of permanent oscillations. For $a=$ $3.57 \ldots$, one can observe an infinite number of fixed points with different periodicity and an infinite number of different periodical cycles; there also exist innumerable combinations of initial conditions from which completely a-periodical, although bounded, trajectories begin; it is from this threshold that the chaotically region begins. For $a>4$, the model explodes.

Introducing the values of parameter a derived from the discrete-time analysis, we obtained the following system of relations:

$$
\begin{aligned}
& m(x)=-x^{2}+x \\
& a(x)=1 /(1-x)
\end{aligned}
$$

Substitute $\mathrm{x}$ between the two equations, the relation among the two parameters can be written as:

$m=(a-1) / a^{2}$

and the graphical representation is shown in Figure 6. On this graph, we can see three remarkable points of parameter $\mathbf{a}$ : for a lower than 1 , the parameter $\mathrm{m}$ becomes negative (that is the value 1 represents the lower limit of parameter $\mathbf{a}$ in case of our model); for parameter a equals value 2, parameter $\mathrm{m}$ has a maximum value (that is, the well-known value 1/4); for parameter a equals value 3 , the function of $m$ has an inflexion point. But from it is known that for parameter a having the value greater than 3 the way to chaos is open. Thus, now we can affirm that for our model solely condition $\mathrm{m}<1 / 4$ is not sufficient. Moreover, it must be doubled by this last restriction on the scale of parameter $\mathbf{a}$. 
Having this new information a deeper analysis on the continuous map of the potential function $V$ demonstrates that for the value $m=2 / 9$ the maximums' branch (curve (C2)) has an inflexion point on the potential surface. This is the image of inflexion point of function $\mathrm{m}(\mathrm{a})$ in the potential function representation (in our graphical representation this inflexion point on $\mathrm{V}$ surface does not appear too clear). Only introducing parameter $\mathbf{a}$ in analysis permitted its discovery. So, a rise of unemployment within $S$ in the proximity or over a value of $2 / 3$ provokes firstly multiple-cycles and then emergence of a chaotic behaviour of the system. A similar situation occurs when the share of inflation within $S$ equals the value $2 / 3$.
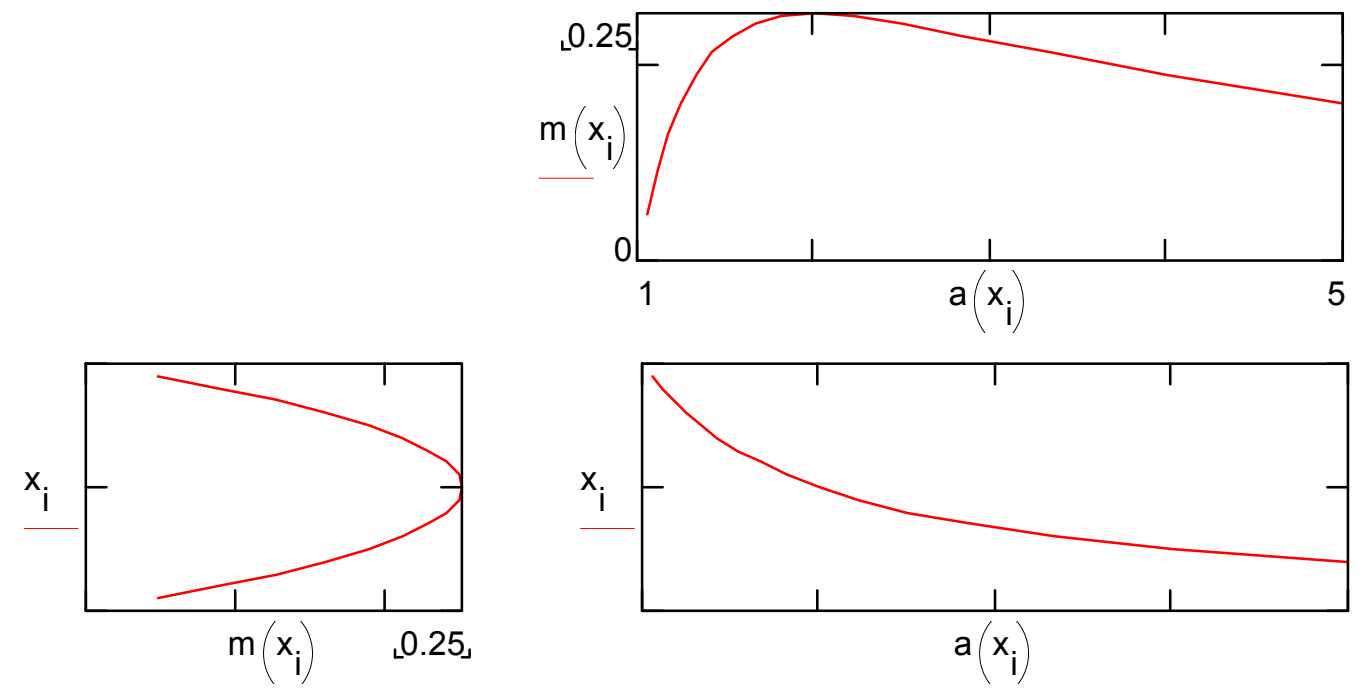

Figure 6

Certainly, considering new parameters can further refine the analysis based on the simple model. Moreover, we note that in many cases the behaviour of the model in discrete-time version may be different comparing with its continuous version. Unfortunately, new developments introduce complicated deformations in the model, new non-linearity, and surely the model must be fundamentally changed. But the solution will be also to analyse the relations and variables grouped separately by considering simultaneously only two or, under special conditions, three of them. In terms of the models derived from our existing economic theory this is the manner of restriction [2]. Our results may be considered as an interlocution in the chaos/chance debate. In fact, the non-linear estimate is one that is confirmed in several fields. But the irregularities are explained by the stochastic, not the deterministic component, inasmuch as the control parameter reaches either the chaotic or the oscillatory bifurcation.

In Annex 2a are presented the estimates for parameter a (only the largest values "a2" are significant for our model) in case of unemployment-inflation plan for some western economies and in Annex $2 b$ the situation in selected eastern countries. The figures represent the estimate values for the period 1970-1996 in case of the first group of countries and for the period 1990-1996 in case of the second group. On graphic representations the interval that signifies the transition from stability to chaos is delimited by the values $\mathrm{aL} 1=3$ and respectively $\mathrm{aL} 2=4$. 
We can conclude, at this stage of the paper, that, when the estimated value of $\mathbf{a}$ is within the chaotic zone, the strain in economic system is larger. As we stressed the symptoms of strain are unemployment and inflation. In the Western countries there was a specific transition during the last 25 years: unemployment replaced inflation as the main symptom of strain. Moreover, it would seem that such long periods of experiencing high unemployment might have infused a larger acceptance by the system of relative large unemployment. This must be viewed in a direct connection with a continuous development of social security programmes in western countries. But such an inference needs to be buttressed by judging the evolution of other variables such as: the dynamic of budget deficits and their sustainability, number of strikes, etc. In Eastern countries it seems that the acceptance of large unemployment is smaller at least during the present transition period. Moreover, in these countries the development of social security programmes is only in an incipient phase. It should be underlined, nonetheless, that an increasing underground sector developed as a valve for absorption of a large part from open unemployment.

For Romania, the trends for unemployment and respectively for inflation are presented in Figure 7, where monthly data cover the period 1990-1995. Also, in Figure 8 is presented the estimated evolution of parameter a for the unemploymentinflation plan. Here the monthly inflation rate was replaced by a yearly equivalent rate. Trends can be observed that seem to be toward the long-run trends in other European countries, but more relevant continue to be the proper yearly evolution, presented in Annex 2b, where the situation looks different. However, a special research would be necessary to evaluate the distance from the situation existing in other economies and from the minimum levels requested by the integration within European Union.

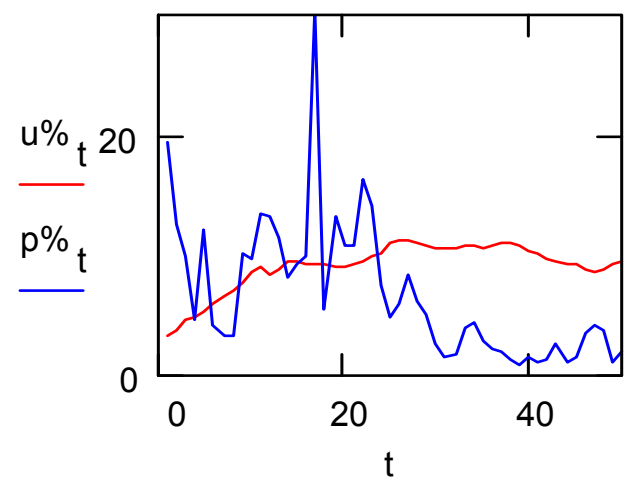

Figure 7

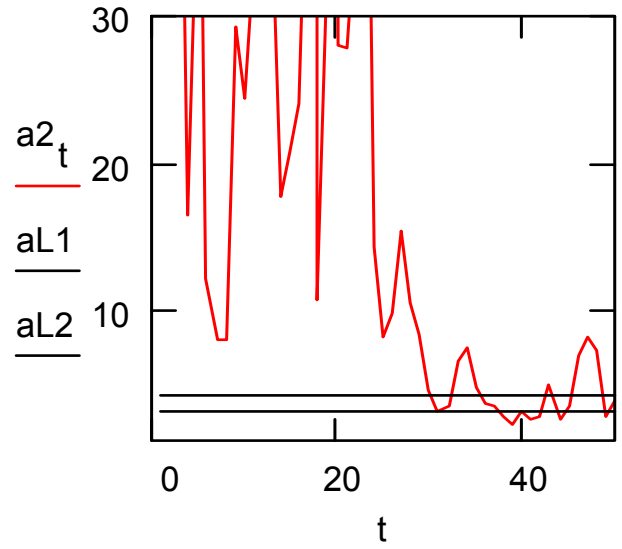

Figure 8

\section{4. Fractal dimension}

Another methodology to estimate trends in economic series and to appreciate their type is that based on fractal dimension. In our study we chose the method called Hurst exponent. According to statistical mechanics, the Hurst exponent $(\mathrm{H})$ should equal 0.5 if the series is a random walk. In other words, the 
range of cumulative deviation should increase with the square root of time. When $\mathrm{H}$ differed from 0.5, the observations were not independent. Each of these carried a "memory" of all the events that preceded it. This is not a short-term memory, which is often called Markovian. This memory is different: it is long-term. More recent events had a grater impact than distant events, but there was still residual influence. On a broader scale, a system that exhibits Hurst statistics is the result of a long stream of interconnected events. Time is very important. Inclusion of a time arrow is not possible in standard econometrics, which supposes series are invariant with respect to time [3].

There are three distinct intervals for the Hurst exponent: (1) $\mathrm{H}=0.50$, (2) $0<$ $\mathrm{H}<0.50$, and (3) $0.50<\mathrm{H}<1.00$. First case denotes a random series. Events are random and uncorrelated. The present does not influence the future. Its probability density function can be normal curve. The standard statistics assume that nature follows the normal distribution, but $\mathrm{H}$ is typically greater than 0.5 for numerous series. The second type of system is an antipersistent, or ergodic, series. If the system has been up in the previous period, it is more likely to be down in the next period. In the third case we have a persistent, or trend-reinforcing, series. If the series has been up (down) in the last period, then the chances are that it will continue to be positive (negative) in the next period. The closer $\mathrm{H}$ is to 0.5 , the noisier it will be, and the less defined its trends will be. Persistent series are fractional Brownian motion, or biased random walks. The strength of the bias depends on how far $\mathrm{H}$ is above 0.50 . Persistent time series are plentiful in nature, as are probably many economic time series. Persistent time series are fractal because they can also be described as fractional Brownian motion. The Hurst exponent describes the likelihood that two consecutive events are likely to occur. Because each point is not equally likely (as it is in a random walk), the fractal dimension of the probability distribution is not 2; it is a number between 1 and 2 . Mandelbrot (1972) has shown that the inverse of $\mathrm{H}$ is the fractal dimension. Note that a random walk is truly 2-dimensional and would fill up a plane.

To estimate $\mathrm{H}$ and fractal dimension for the plan u-p and for global surface (u-p-y), we propose the following dynamic series:

$$
\begin{aligned}
& \text { doup }_{t}:=\sqrt{\left(u \%_{t}\right)^{2}+\left(p \%_{t}\right)^{2}} \\
& \text { do }_{t}:=\sqrt{\left(u \%_{t}\right)^{2}+\left(p \%_{t}\right)^{2}+\left(y \%_{t}\right)^{2}}
\end{aligned}
$$

In Annex 3 we present some applications obtained by using an own methodology (Daianu and Albu, 1996). 


\section{Local bifurcations in continuous-time dynamical systems and transitions in European countries}

\subsection{Using the potential functions to spatial representation of inflation-unemployment relationship}

Every time, in economic literature the graphical representation of models is very useful to understand the intimate relations between variables. Till the PCs era and the development of some adequate software, only the graphical representation in 2-D was disposable for economists. A decisive role in the achievement of some sophisticated software to build 3-D representation of our models had the so-called theory of the potential-functions. Today, it is also integrated within the Catastrophe Theory, or more general Theory of Structural Stability, that cover the continuoustime models. At its turn, this high-level theory is going to be integrated with the socalled Deterministic Chaos Theory, constituting together a mega-theory named the General Theory of Discontinuities.

In this section of the paper, we present how it looks in 3-D the time-evolution of relation between inflation and employment and the so-called contour-plot map in some European countries. These pictures are very useful to understand how they are the long-run trends and to obtain preliminary information about the existence of some eventually attractors. We build all the graphs with the support of the theory of potential functions and using the MathCAD-Professional 6 Plus soft.

First group of graphs represents the dynamics in the three-dimensional space of the relation: rate of GDP growth (y) - GDP per capita, in constant prices (ylc) - unemployment rate (x). They are presented in figures from Annexes $4 a, 4 b$, and $4 \mathrm{c}$ for three western countries and, respectively, in Annex 5 for five eastern countries. On the potential-surface we can see some small black zones, which means the existence of attractors. For instance, in case of Belgium (Annex 4a), there are three such zones around the points of coordinates $(5.0 ; 14.0 ;-1.5),(10.8$; $16.0 ;-0.9)$, and respectively $(13.0 ; 19.5 ;-1.7)$. Also, we can identify the years when they are occurred: 1975-76, 1981-82, and respectively 1993-94. The significance of these remarkable points is that they are points of some fundamental changes of trends. The evolution below and the evolution after these points are quite different. One important conclusion for western countries is that the unemployment level that corresponds to an attraction zone, i.e., the stabilisation level, is growing once the GDP per capita level is increasing. In case of eastern countries, due to the short period (the graphs in Annex 4 are based only on the data for period 1990-95) within one free-market economy system, it is difficult to conclude. However, we can affirm that the sign of one more structured economy is the occurrence of the mentioned attractors already existing in western countries.

Second group of graphs refers to the dynamics in the three-dimensional space of the following relation: rate of GDP growth $(y)$ - GDP per capita, in constant prices (ylc) - yearly inflation rate (z). They are presented in figures from Annexes $6 a, 6 b$, and $6 c$ for three western countries and, respectively, in Annex 7 for five eastern countries. In case of western countries, the conclusion is that the attractor in case of inflation is situated at one smaller level, even near zero value. In contrast with this situation, in eastern countries the mentioned attractor seams to be placed at very high level of inflation.

In order to achieve a more rigorous analysis we called the last results obtained within the integrated theory of bifurcations. 


\subsection{The prototype of the pitchfork bifurcation and its application to investigate the unemployment dynamics}

Coming from the empirical data on the evolution of the unemployment in the western countries in last 25-30 years and from the hypothesis on the natural rate of unemployment existing in literature, we conceived a model that can be described by the following first-order differential equation:

$\mathrm{dy} / \mathrm{dt}=\alpha[\mathrm{xn}(\mathrm{y})-\mathrm{x}(\mathrm{y})]$

where, $y$ is the GDP per capita, xn - "natural" unemployment, $x$ - actual unemployment, and $\alpha$ - a coefficient. Let ycr be the "natural" or "critical" level of GDP per capita, and formulate (3.1) in terms of the deviations from the appropriate $\mathrm{xcr}$ and $\mathrm{xncr}$ levels (we chose $\mathrm{xcr}=\mathrm{xncr}$ ):

$\mathrm{dY} / \mathrm{dt}=\alpha[\mathrm{Xn}(\mathrm{y})-\mathrm{X}(\mathrm{y})]$

with $Y=y-y c r, X n=x n-x n c r$, and $X=x-x c r$. Also, the following relations between $X$ and $Y$ and respectively between $X n$ and $Y$ were initial supposed:

$X(Y)=a Y+b$

$X n(Y)=A Y^{3}+B Y^{2}+c Y+D$

where, $a, b, A, B, c$, and D are coefficients. So, in points where the two curves are intersecting will be achieved the natural level of $y$. The described system seems to be like one that has behaviour of type pitchfork bifurcation. However, taking into account the condition of an odd function for $d Y / d t=f(Y ; \mu)$ with respect to $Y$, i.e., $f(Y ; \mu)=-f(-Y ; \mu)$, we chose the solution $B=0$, and $D=b$. So, the equation (3.4) was replaced by:

$X n(Y)=A Y^{3}+c Y+b$

Now, after some operations, the expression of function $f$ can be written as:

$f(Y ; \mu)=\beta\left(\mu Y-Y^{3}\right)$

with $\beta=-\alpha / A$ and $\mu=(a-c) / A$.

Define $\mu_{0}$ as that parameter value for which the eigenvalue of (3.1) or in the new form (3.4) is zero, i.e., $\partial f\left(y^{*}, \mu_{0}\right) / \partial y=\lambda=0$. Then, the conditions of the existence theorem are fulfilled and a pitchfork bifurcation occurs at $\mu_{0}$. The existence theorems for the main types of bifurcations are presented in Annex 8.

We applied this model to the case of Belgium, but using three different sets of critical points and $\mu_{0}$ parameters. The main conclusion is that for greater level of GDP per capita the impacts of unemployment and respectively that of the natural unemployment on the economic growth (represented by changes in GDP) are changing in sense that the natural unemployment response to the deviation from the natural GDP level is getting smaller. Coming from a point, at a high level of GDP per capita (around 20,000 dollars/inhabitant, in case of Belgium), the structure of the relations between unemployment, in general, and the increasing rate of production (GDP) changed dramatically. From that point, the dynamics of 
unemployment and natural unemployment became smaller and smaller related to the GDP dynamics, their evolution being more autonomous, governed by different own lows. However, the conclusion must be certificate by application on huge statistical data. Unfortunately, the achievement of one more efficient methodology still remains for our future research. The dramatic changes evaluated by the model are suggested graphical in Figures 9 and 10 (where the annual change of GDP, is denoted by vy and GDP per capita by ylc).
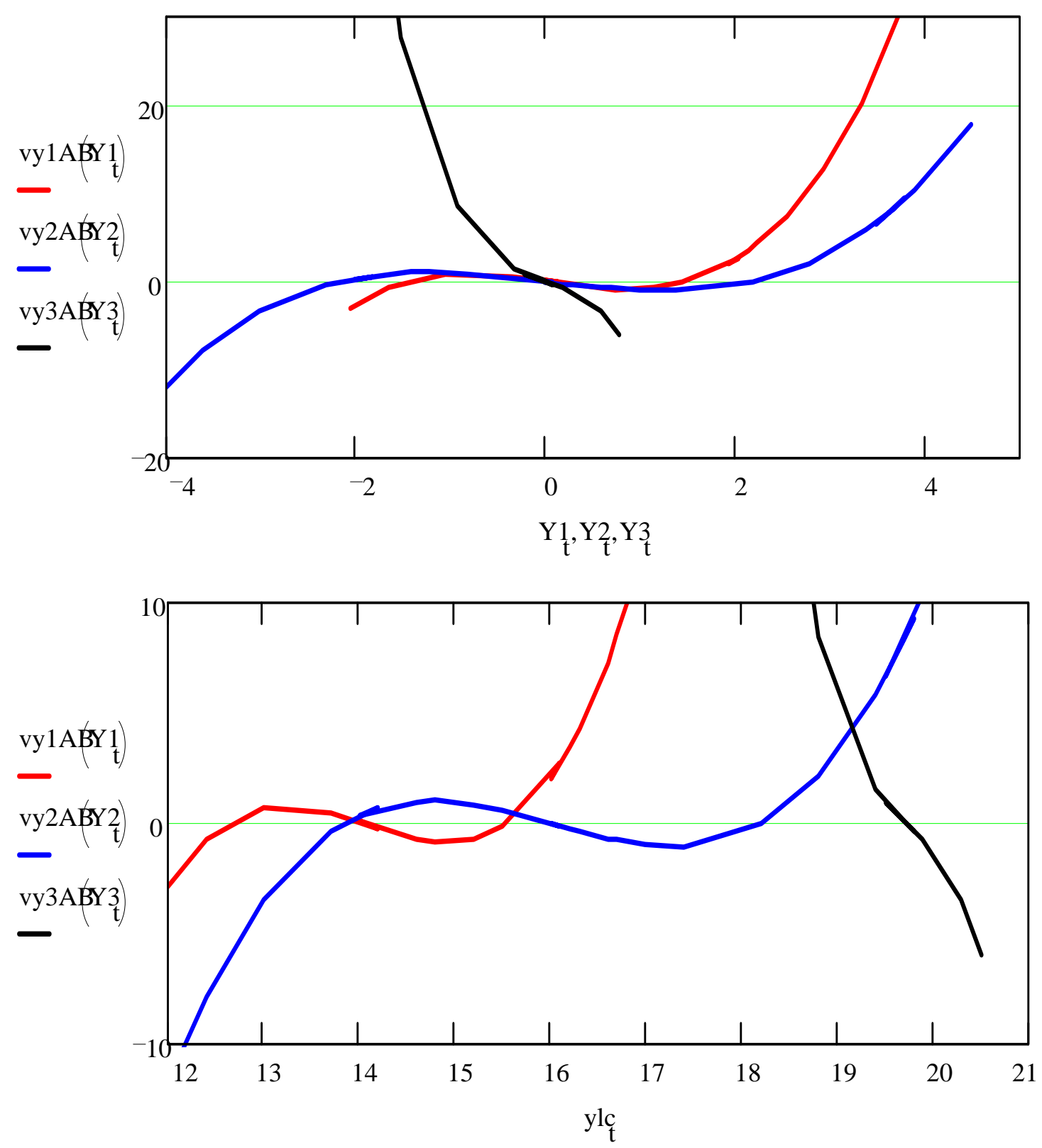

Figure 9 

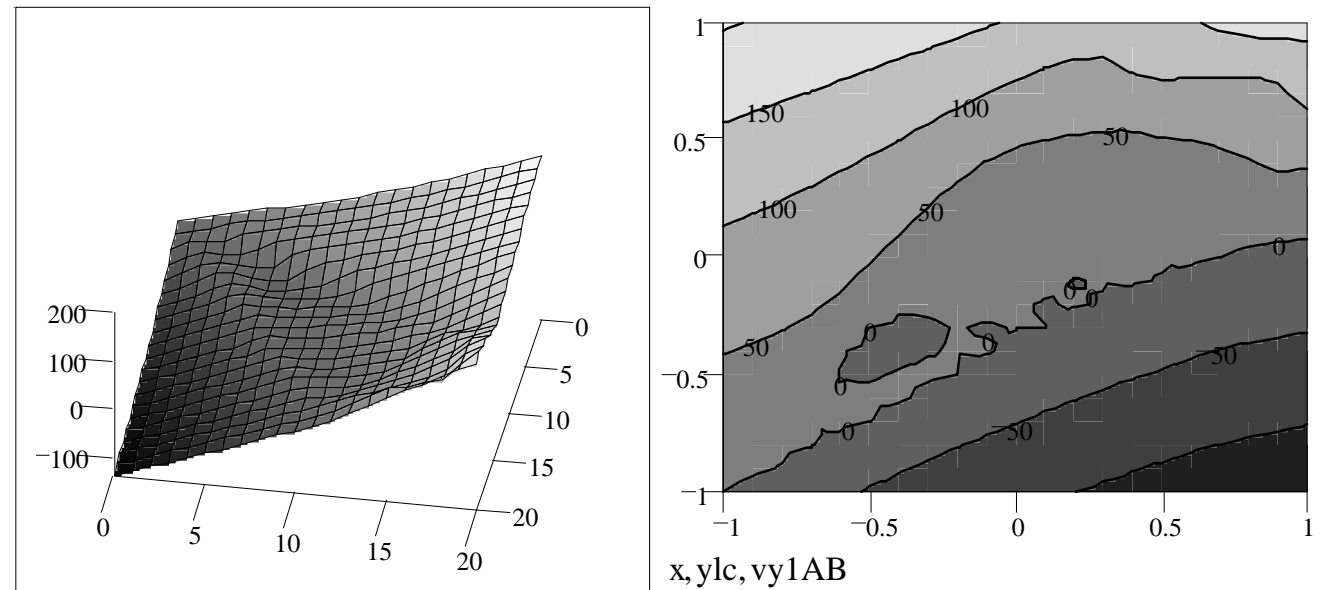

x, ylc, vy1AB
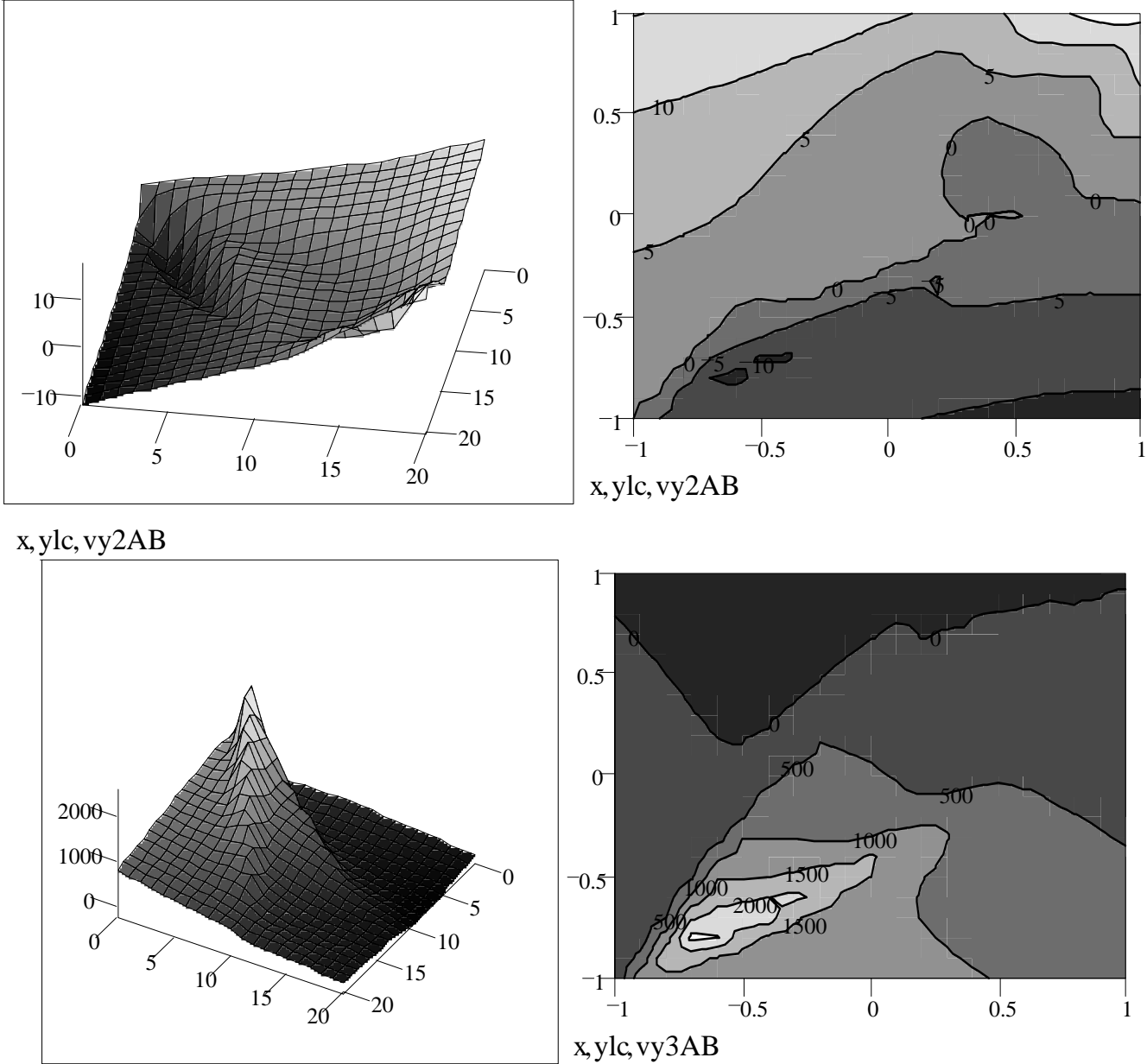

x,ylc,vy3AB

Figure 10 


\subsection{A truncated version of the pitchfork bifurcation model and applications}

As a continuation of the model presented in section 2.3 , we present here a kind of pitchfork bifurcation, in sense that while the conditions of theorem A8.3 (from Annex 8) for a pitchfork are fulfilled, including that of odd function, the condition to remain within the unit interval $[0 ; 1]$ is violated. However, the applications of the model in case of some countries permitted to capture several important features during a medium or even long transition.

Let $z(x)$ be the general three-order equation of inflation with respect to unemployment $(\mathrm{x})$, where $\mathrm{z}$ and $\mathrm{x}$ are evaluated in terms of shares within the mentioned sum $\mathrm{S}$

$z(x)=a x^{3}+b a x^{2}+c x+d$

and the following three additional relations between coefficients: $d=1 ; a=2(c+2)$; and $b=-3(c+2)$. These relations permitted to define other function $z^{*}$ as function of $x$ and the parameter $\mathrm{C}$

$z^{*}(x ; c)=2(c+2) x^{3}-3(c+2) x^{2}+c x+1$

The function $f$ resulting from the difference between (3.7) and (3.6) and written that $z(x)=1-x$ is as following:

$f(x ; c)=2(c+2) x^{3}-3(c+2) x^{2}+c(x+1) x=0$

and it would be viewed as potential surface. Also, using the same procedure we write the so-called potential function from which is derived $f(x ; c)$

$V(x ; c)=\left\{\left[(c+2) x^{4}\right] / 2\right\}-(c+2) x^{3}+\left[(c+1) x^{2}\right.$

The remarkable values of $c$ are -4 and -2 . Thus, in fact, there are two parameters $c^{*}$ and $\mathrm{c}^{* *}$ that are governing the dynamics of the system.

We applied the model to the data for Belgium, France, UK, and Romania. The results were too different, showing firstly if an economy is structured or not. In Figure 11 are presented the graphs of potential function against unemployment for the yearly data in period 1970-96 for the three western countries and for monthly data among 1990-96 for Romania. Probably, more interesting are the graphs representing the time-evolution presented in Annexes 9, when we can identify the hot moments and their effect on the stability plan. The form of curves changed when the slow parameter $c$ varies. On the first group of graphs, the change from curves having one non-stable equilibrium to ones having a stable equilibrium and a large zone of attraction around this means the transition from older periods to the actual period. The type of curves is changing fundamental, in western countries when parameter $c$ has a transition from positive values to negative values. The largest shocks are very well presented on graphs and also the bifurcation set as well as. The trend is clear in case of western countries; it means the transition to a more stable and quite zone, with less strain, while in eastern countries, like in Romania in this example, the restructuring of economy remains to be achieved. A general spatial picture can be viewed in the graphs where both the shares of inflation and unemployment are plotted together with the $\mathrm{c}$ parameter. The curvature of spatial lines and the intensity of plotted colours are correlated with the strain in the system (Figure 12). 

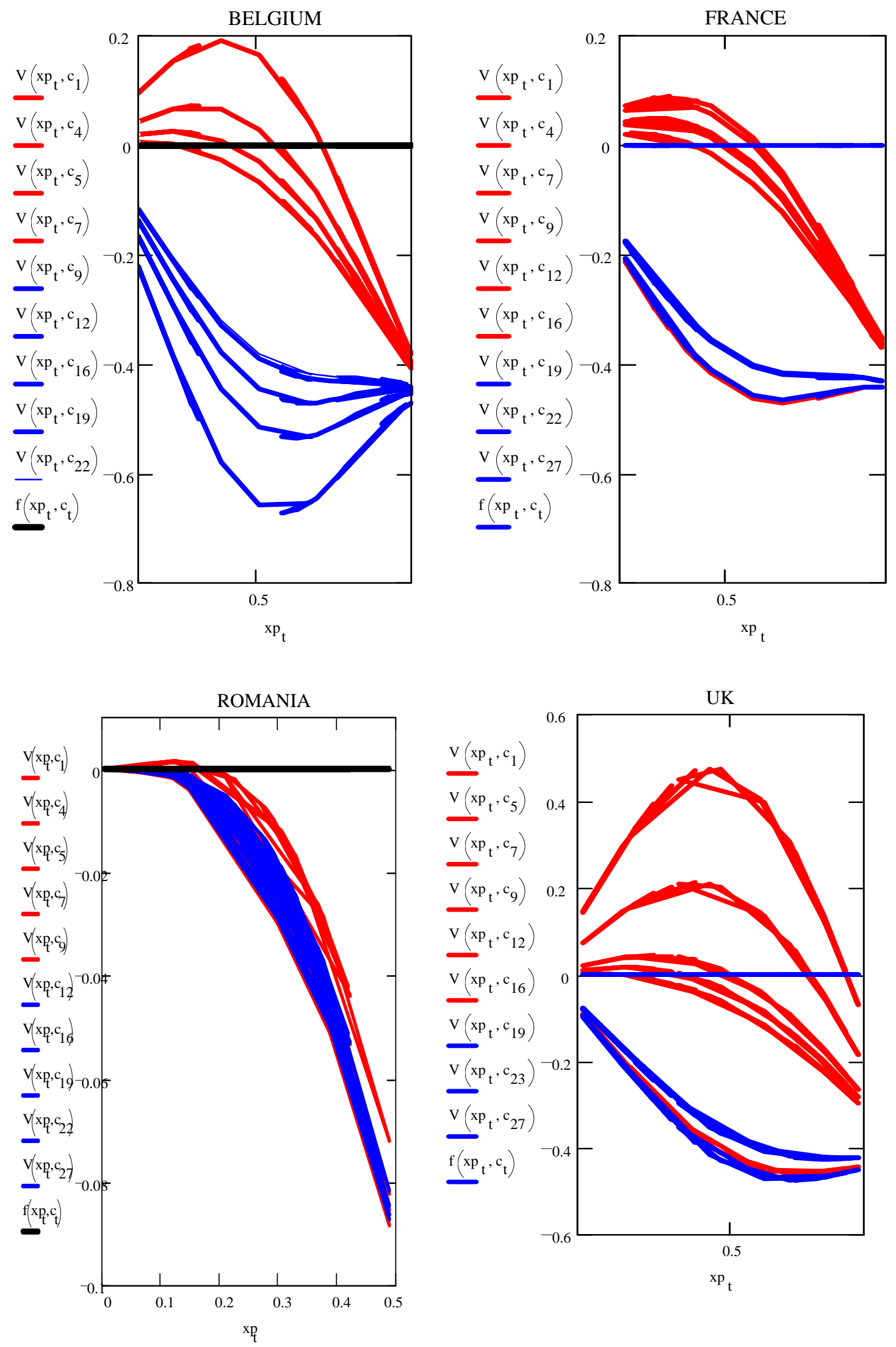

Figure 11 

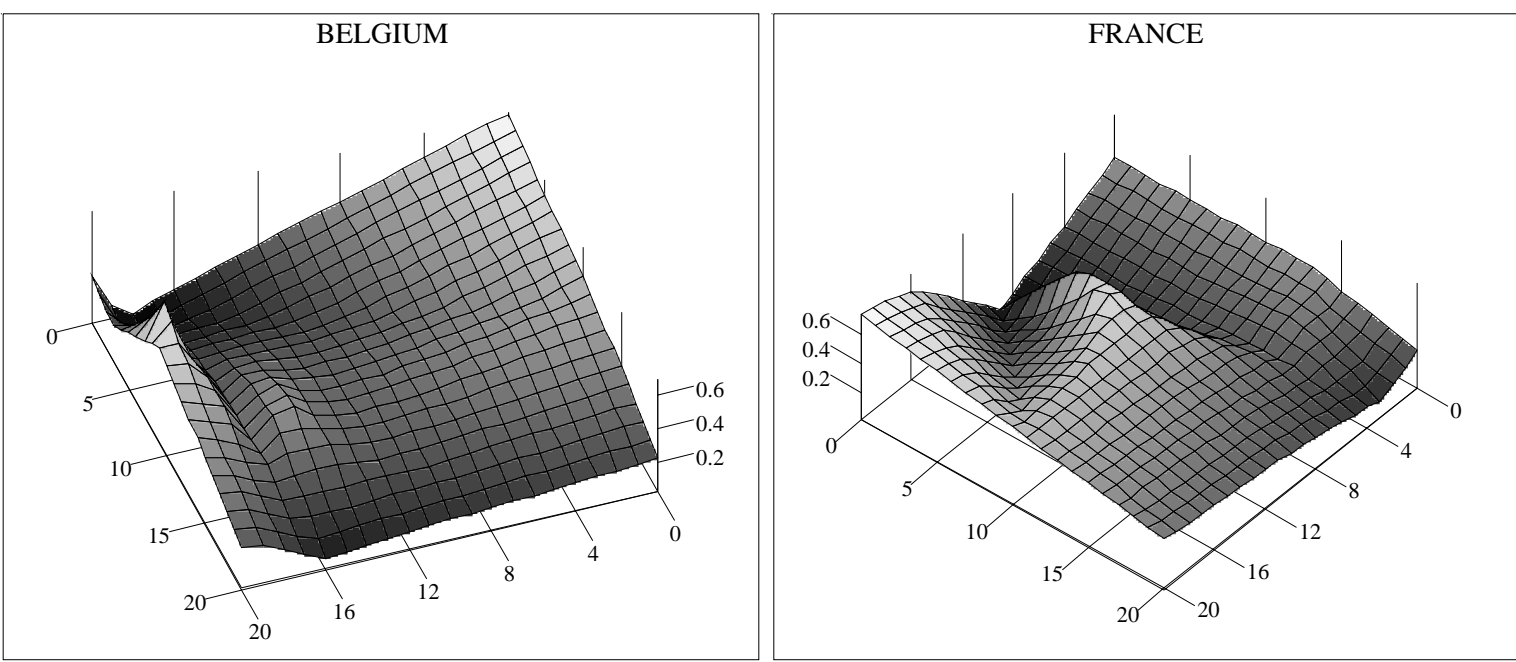

c, xp, zp

c, $x p, z p$
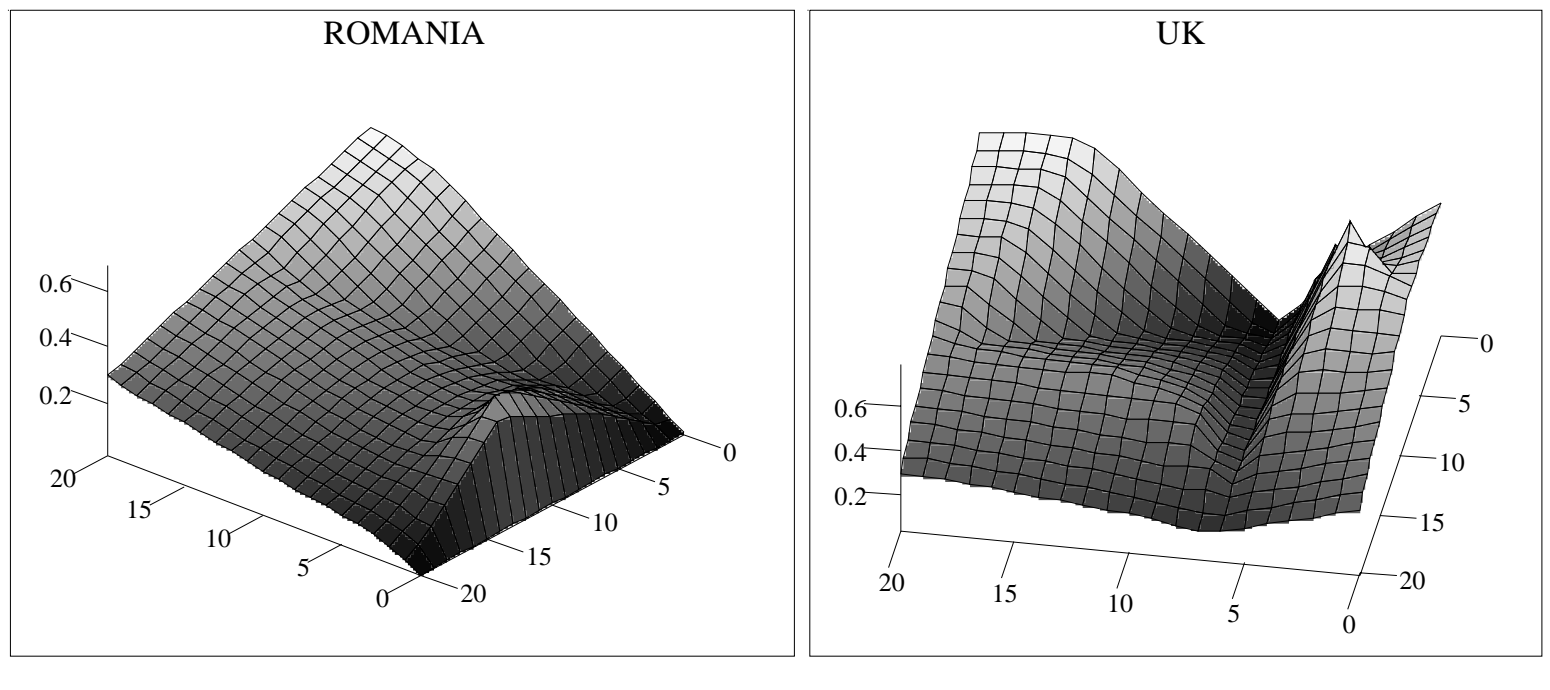

c, zp, xp

c, zp, $x p$

Figure 12 


\section{Global bifurcations and spiral-type chaos in an inflation-unemployment cycle model}

\subsection{Theoretical context}

In connection with the Shilnikov theorem [4] on the fulfilment of the local conditions of stability and the demonstration of presence of a homoclinical orbit it was specified that some specific dynamical systems are known which posses a homoclinical orbit and allow the fulfilment of the local stability proprieties of the Shilnikov theorem to be easily verified. In a series of papers [5] it was demonstrated that the following two-differential-equations dynamical system

$$
\begin{aligned}
& d^{2} x / d t^{2}+a(d x / d t)+x=z \\
& d z / d t=f_{\mu}(x),
\end{aligned}
$$

or, written as a third-order differential equation,

$$
d^{3} x / d t^{3}+a\left(d^{2} x / d t^{2}\right)+d x / d t=f_{\mu}(x)
$$

with a as a constant, exhibiting chaotic behaviour for appropriate forms of the oneparameter family of functions $f_{\mu}(x)$.

For instance, the specification $f_{\mu}(x)=\mu x(1-x)$, i.e., a logistic function, like that which we used already in the section 2.3, yields geometrical objects that resemble the diverse Rössler attractors. The motion is characterised by a screwtype or spiral-type structure depending on the magnitude of the parameter $\mu$ [6]. Other forms of the function $f_{\mu}$ with similar non-invertibilities lead to comparable results (Lorenz, 1989). A graphical representation of Rössler model can be found in Figure 13, obtained by using an own algorithm to transform the differential-equation model into one model with finite differences.

\subsection{Spiral-type chaos in a specific cycle of inflation-unemployment relation}

In this part of paper we present a very simple economic example of the emergence of a chaotic motion in three-dimensional systems analogous to the case presented above. We where inspired by the modified macroeconomic business cycle model with inventories which in its discrete-time version was first approached by Metzler (1941). Also, many improvements were coming from the Mundell's analyse within his so-called supply-side economics (1990). Our model is very closed to that studied by Gandolfo (1983) and presented by Lorenz (1989).

Let $y$ denote the GDP and assume that output adjust according to discrepancies between the desired (or expected) and actual inflation rate, i.e.,

$\mathrm{dY} / \mathrm{dt}=\alpha[\mathrm{Xn}(\mathrm{t})-\mathrm{X}(\mathrm{t})], \alpha>0$,

with $\mathrm{Xn}(\mathrm{t})$ as the natural and $\mathrm{X}(\mathrm{t})$ as actual unemployment at $\mathrm{t}$. We suppose also that the actual unemployment changes when disequilibria prevail on the goods market, i.e., on the inflation-side

$(\mathrm{dX} / \mathrm{dt})(\mathrm{t})=\lambda[\mathrm{Z}(\mathrm{t})-\mathrm{Zn}(\mathrm{t})], \quad \lambda>0$, 
where $Z(t)$ is the actual inflation and $Z n(t)$ - the expected rate of inflation, respectively. The natural unemployment is assumed to depend linearly on the expected output, $Y^{e}(t)$, in $t$

$\mathrm{Xn}(\mathrm{t})=\mathrm{h} \mathrm{Y}^{\mathrm{e}}(\mathrm{t}), \mathrm{h}>0$,

implying that

$(d X n / d t)(t)=h\left[\left(d Y^{e} / d t\right)(t)\right]$
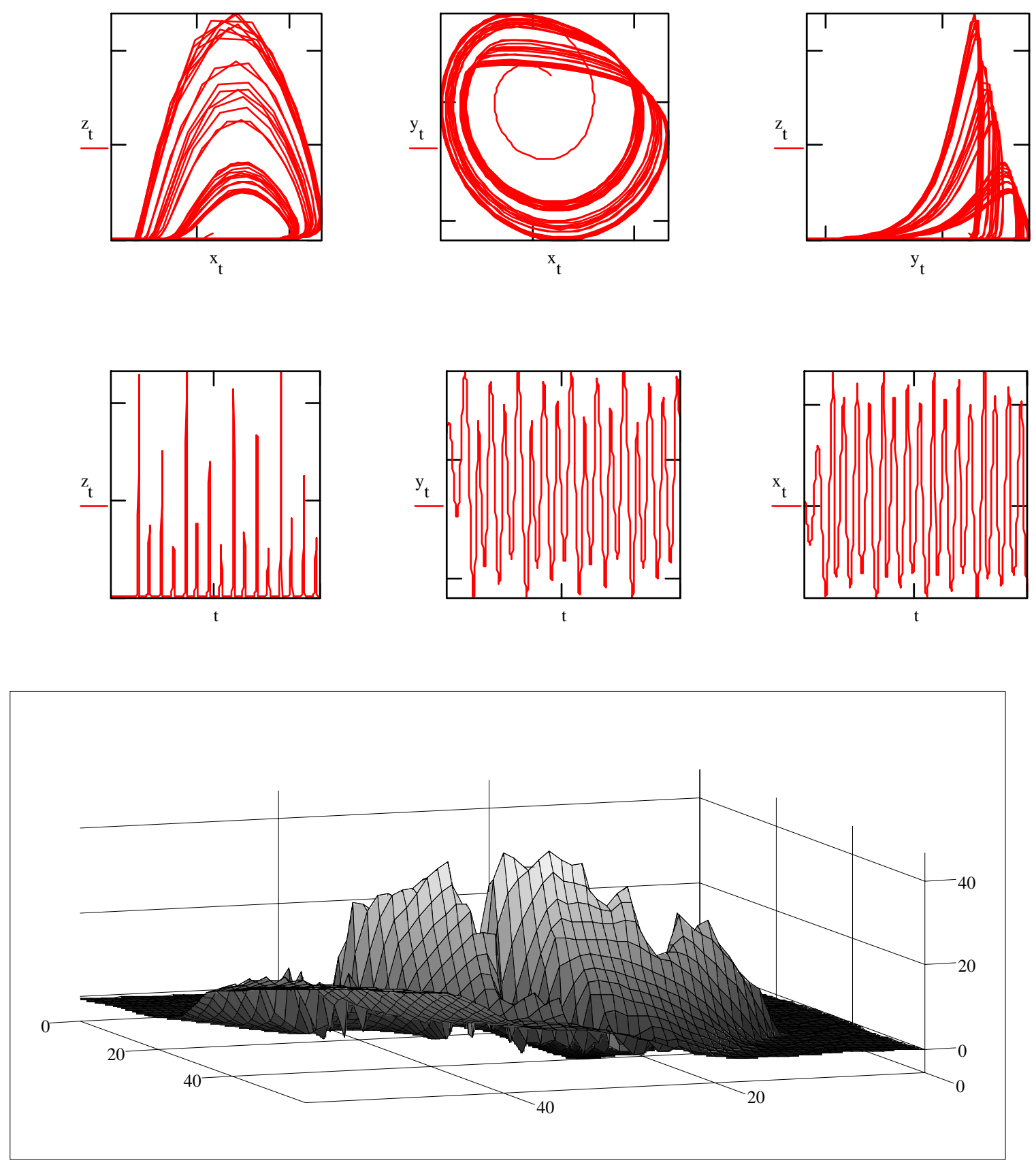

$\mathrm{x}, \mathrm{y}, \mathrm{z}$

Figure 13 
The expected output is determined according to a modified hypothesis of adaptive expectations which considers only the rate of change of current output but which also includes the changes in this rate:

$Y^{e}(t)=Y+k 1[(d Y / d t)(t)]+k 2\left[\left(d^{2} Y / d t^{2}\right)(t)\right]$

Thus, expected output changes according to

$\left(d Y^{e} / d t\right)(t)=[(d Y / d t)(t)]+k 1\left[\left(d^{2} Y / d t^{2}\right)(t)\right]+k 2\left[\left(d^{3} Y / d t^{3}\right)(t)\right]$

Differentiating (4.3) with respect to time and substituting for $[(\mathrm{dXn} / \mathrm{dt})(\mathrm{t})]$ and $[(d X / d t)(t)]$ yields the third-order differential equation

$\left[\left(d^{3} Y / d t^{3}\right)(t)\right]+\{[\alpha h k 1-1] /(\alpha h k 2)\}\left[\left(d^{2} Y / d t^{2}\right)(t)\right]+(1 / k 2)[(d Y / d t)(t)]=$

$$
=[\lambda /(a k 2)][Z(t)-Z n(t)]
$$

or, abbreviated,

$\left[\left(d^{3} Y / d t^{3}\right)(t)\right]+K 1\left[\left(d^{2} Y / d t^{2}\right)(t)\right]+K 2[(d Y / d t)(t)]=\beta[Z(t)-Z n(t)]$,

with $\beta=\lambda /($ a k2 ).

Gandolfo (1983) demonstrated that a equation like (4.10) is unstable when $Z(t)$ is a linear function of output, e.g., in our case, $Z(t)=(1-c) Y(t)-Z 0,1 \geq c>0$, when $\mathrm{Zn}$ is autonomous, i.e., $\mathrm{Zn}(\mathrm{t})=\mathrm{ZnO}, \mathrm{ZnO}>0$, and when $\mathrm{K} 1<0$.

The linearity of the inflation rate and expected inflation functions is assumed only for technical convenience. However, there is no convincing reason why these linear functions should constitute the only economically relevant forms. Once the linearity assumption is abandoned, it can be shown that the modified model has the form (4.2) with a noninvertible function $\mathrm{f}_{\mu}(\cdot)$.

Define $y^{*}, z n^{*}, z^{*}, x^{*}$, and $x^{*}$ as the equilibrium values of output, expected and actual inflation rate, unemployment and natural unemployment, respectively, and consider the deviation from these equilibrium values, i.e., $y=Y-y^{*}, z n=Z n-Z^{*}$, $\mathrm{z}=\mathrm{Z}-\mathrm{Z}^{*}, \mathrm{x}=\mathrm{X}-\mathrm{x}^{*}$, and $\mathrm{xn}=\mathrm{Xn}-\mathrm{xn}^{*}$.

Equation (4.10) then becomes

$\left[\left(d^{3} y / d t^{3}\right)(t)\right]+K 1\left[\left(d^{2} y / d t^{2}\right)(t)\right]+K 2[(d y / d t)(t)]=\beta[z(t)-z n(t)]$

Assume that both actual and expected inflation are nonlinear function of output. Possible shapes of the functions that exhibit two points of intersection of the actual inflation and expected inflation can be supposed. The difference $(z(y)-z n(y))$ therefore describes a one-humped curve similar to the logistic function $f_{\mu}(x)=\mu x$ $(d-x)$, used by Ameodo et al. (1981) for the case $d=1$.

Assumption 4.1

(i) $\mathrm{K} 1>0$ and $\mathrm{K} 2$ close to unity.

(ii) $\beta\left[z_{\mu}(y)-z n_{\mu}(y)\right]$ is one-humped function $f_{\mu}(y)$ with a critical value 
$\mathrm{y}_{\mathrm{cr}}>0$, the slope of which can be controlled by a single parameter $\mu$.

Under Assumption 4.1, our model (4.11), also being similar to the Metzlerian model, is nearly identical with equation (4.2). The Lie derivative (the divergence) of (4.11) is negative because of $\mathrm{K} 1>0$ [7]. The system is therefore volume contracting and possesses an attracting invariant set. The dynamic behaviour of (4.11) is not essentially different from that of (4.2) and it can see that (4.11) possesses a Shilnikov-type structure for the assumed values of $\mathrm{K} 1, \beta$, and the slope of the excess supply function. In contrast to logistic, one-dimensional difference equations, rather flat shapes of the one-humped curve are sufficient to encounter chaotic motion (Arneodo et al., 1982). It can be expected that several other modifications of the model are possible which still imply the emergence of a Shilnikov-type attractor when the excess supply function is noninvertible. The main remaining problem, including for our future research, is how we should capture these types of attractors from the actual set of existing statistical data.

\section{A discrete-time non-linear model to investigate transitions to chaos in a modified Phillips curve system}

As we showed in section 2.2, the stagflation phenomenon was relatively recent added to the problematic of inflation-unemployment relationship (Santeremo and Seater, 1978). Also, we mention that early empirical investigations indicated an inverse non-linear relationship (Phillips, 1958; Lipsey, 1960). An attempt to model the stagflation phenomenon has included through introduction of some additional factors in explaining actual inflation, such as the expected inflation rate (Friedman, 1968). One more complete approach to model the stagflation is provided by Fischer and Jammernegg (1986) who conceived a dynamical system based on the catastrophe theory approach (Lorentz, 1989). Among studies that incorporated the Phillips curve we mention here those of Goodwin (1967), Pohjola (1981), and Soliman (1996).

Our model consists in the following system of three equations:

$$
\left[\begin{array}{c}
\pi_{t} \\
\pi e_{t+1} \\
u_{t+1}
\end{array}\right]:=\left[\begin{array}{c}
g\left(u_{t}\right)+h 1 \cdot \pi e_{t} \\
\pi e_{t}+h 2 \cdot\left(\pi_{t}-\pi e_{t}\right) \\
-h 3 \cdot\left(\pi e_{t+1}-\pi_{t}\right)+u_{t}
\end{array}\right]
$$

where $\pi_{t}$ and $\pi e_{t}$ are the actual inflation and expected inflation rates in period $t$ respectively and $u_{t}$ is the level of unemployment in period $t$. The incorporation of inflationary expectations into actual inflation is represented by the parameter $\mathrm{h} 1(0$ $\leq \mathrm{h} 1 \leq 1)$. The second equation expresses the fact that the inflationary expectations are adaptive expectations in our case. The parameter $\mathrm{h} 2(0 \leq \mathrm{h} 2 \leq 1)$ represents the degree that errors made in predicting actual inflation are corrected. The unemployment dynamics is expressed by the last equation of system, which includes the assumption that the next unemployment rate will be influenced by the difference between expected inflation and actual inflation. The extent of this influence is governed by the elasticity of unemployment with respect to real monetary growth, therefore parameter h3 (h3 > 0).

Although there is theoretical justification for non-linear inflationunemployment in literature, there is no agreement with respect to its functional form. In our model we considered the following functional form: 
$\mathrm{g}(\mathrm{u}):=\left(\frac{\mathrm{k} 1}{\mathrm{lcr}^{2}}+\mathrm{k} 2\right) \cdot(1-\mathrm{u})^{2}+\left(-\frac{\mathrm{k} 3}{\mathrm{lcr}}-\mathrm{k} 4\right) \cdot(1-\mathrm{u})+\mathrm{k} 5$

where $\mathrm{k} 1, \mathrm{k} 2, \mathrm{k} 3, \mathrm{k} 4, \mathrm{k} 5$, and Icr are parameters. We mention that this functional form represents the result of another model used by us for the determination of the critical level of unemployment, ucr (ucr = 1 - Icr), under the condition of a CobbDouglas function of production with respect to the employment level.

The simulations obtained with the model demonstrate a very complex dynamics of inflation-unemployment relationship and the existence of attractors in case of some critical values attributed to parameters as well. Also, the great sensibility to the small differences in the initial values is evident. In Figure 14 are presented several functioning regimes in the $\left(\pi_{t}-u_{t-1}\right)$-space varying with two sets of data attributed to parameters, but many others regimes can be extracted from the model simulation. The values of fixed parameters are $\mathrm{lcr}=0.71 ; \mathrm{k} 1=3 ; \mathrm{k} 2=0.8 ; \mathrm{k} 3$ $=6$; and $\mathrm{k} 4=4$. As initial set of values for $\pi$, $\mathrm{u}$, and $\pi \mathrm{e}$, we chose $\pi 0=0.08, \mathrm{u} 0=$ 0.09 , and respectively $\pi \mathrm{e}=0.08$. The parameters that change are $\mathrm{h} 1(0.1 ; 0.2 ; 0.3$; $0.5)$, h2 (0.1;0.3;0.45), and h3 (0.6; 0.5; 0.7; 0.9). 

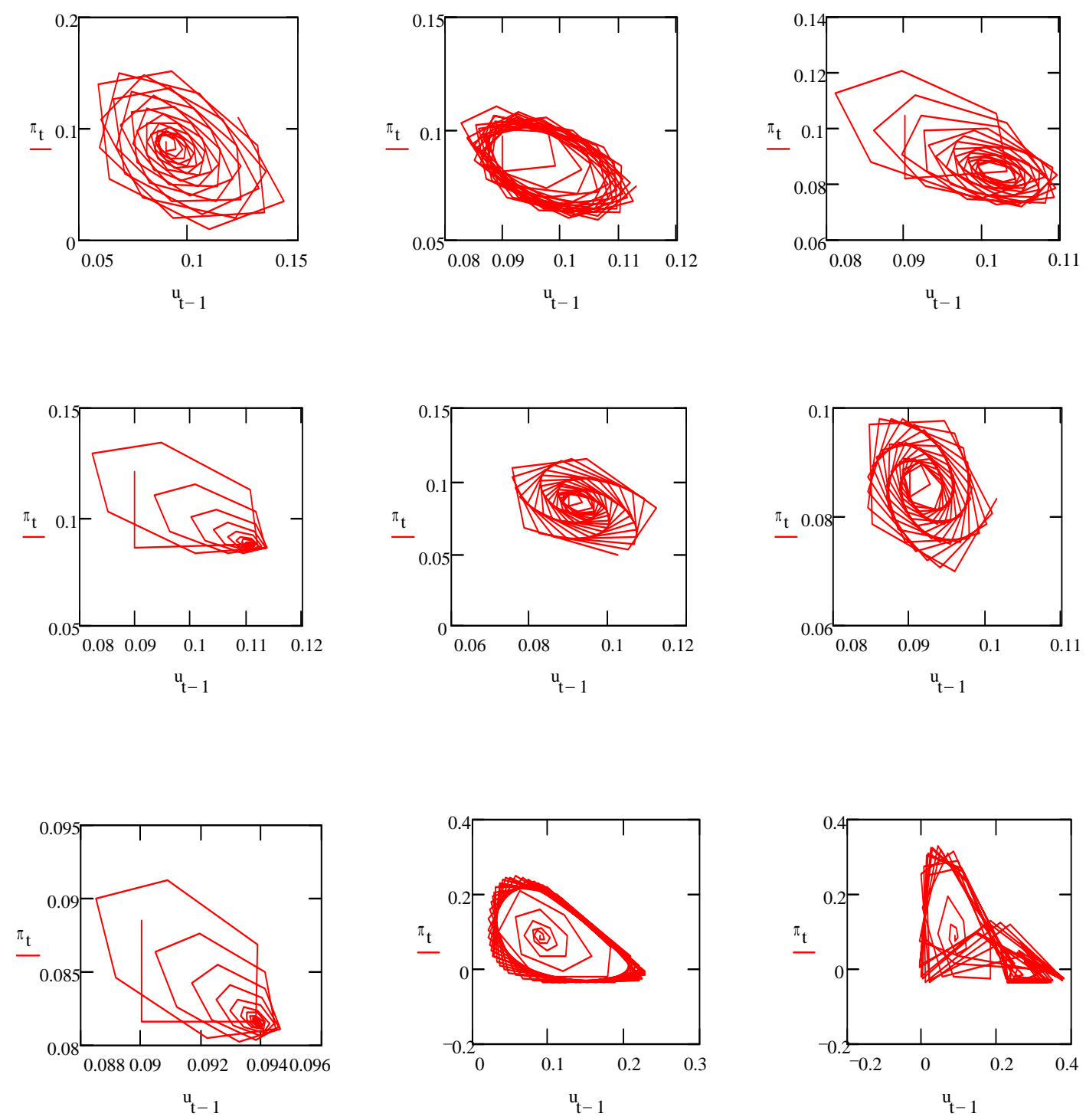

Figure 14 


\section{Conclusions}

In summary we have illustrated several phenomena exhibited by the investigation of the inflation-unemployment relationship and its attached strain induced in economic systems.

Our goal was to demonstrate, and we hope that at least partially we issued, that the actual transition in eastern countries represents only a stage within a longrun wave evolution on the economic development scale. Also, it was demonstrated an relative important transition process including in western countries, namely to a higher natural level of employment and to a period in which the unemployment become more and more autonomous relating to the dynamics of GDP. On the unemployment-side occurred a strong relaxation, higher levels of unemployment being viewed as normal in contrast to the situation on the inflation-side.

On the other hand, in Eastern European countries it seems to exhibit a mirrored-situation: relative small levels of unemployment, relating to the severe structural crisis that is manifesting after 1989, and huge inflation rates. Despite of these differences the signs of an imminent converging process were emerged. To accelerate the process, the immediate and urgent solution, especially for countries as Romania, is to finish the great privatisation doubled by a huge effort oriented to one process of profound economic restructuring. In terms of the inflationunemployment relationship this means higher unemployment and controlled decreasing inflation. In such hot period it is important that the government to benefit of the population support and in Romania this is the case after November 1996.

Important for the actual transition in eastern countries is that the converging process does not suppose necessarily a repetition of the western evolution coming from the '60s. In chapter two is suggested that changing the parameters of curves in the inflation-unemployment -space (that reflects in fact the structural changing) can be assured a more rapid transition as a veritable jump. A first modality to discover in which zone is placed an economy from the long-run viewpoint is offered by the model derived from the three-degree potential-function doubled by the estimation of fractal dimension.

The bifurcations models facilitate a deeper analysis. Moreover, a pitchfork model like that used in section 3.3 can indicate the actual stage of an economy relating to a so-called non-structured degree. Aside of the traditional methods, a developed model in this sense would be an additional tool for the economic policy makers.

Some promising results seem to emerge from the more complicated models like spiral-type model and respectively Rössler-type model. In this sense, to build variants of models more adapted to include the actual statistical data continuing to remain in our duty for the future research projects. However, this kind of models together with the non-linear discrete-time model of the modified Phillips curve give us, and would have to give to the policy makers, an important alarm-signal, namely that the structure of apparently simple classic models may exhibit a large behavioural menu and that the chaotic regimes can emerge when the actual values of some special parameters are evolving near certain threshold-values. Additionally, the named regimes may remain hidden in the context of some traditional models usually applied by the policy makers. 


\section{Notes}

[1] The modified Phillips curve can explain the simultaneous presence of high inflation and unemployment if inflationary expectations are high. An alternative way of modelling the stagflation phenomenon was provided by Woodcock and Davis (1979) based on the catastrophe theory. Also, Fischer and Jammernegg (1986) applying a cusp model on the US economy obtained better results then in case of the classic model.

[2] The conclusions may be result from our limited possibilities to model, from the limits of our present science and our existing mathematical apparatus, from our own power of understanding reality (the scientists from other disciplines as physics, for instance, had already the power to affirm frankly their own limits in investigating and understanding, but some economists have yet reticence to affirm this). Maybe, the main preoccupation of research would be only to register the evidence of facts, even when they refuse to come into our existent models. Using the recent results of Chaos Theory and its close Fractal Geometry and respectively Catastrophe Theory can develop also the investigation of some economic time series or constructing some models including up to 4 or even 5 slow parameters. But frequently the explanations will be beyond those accepted by standard economic theory. Consequently, the partisans of standard economics will contest many of these studies.

[3] For details see Peters (1991).

[4] Many dynamical systems in continuous time as well as in discrete-time $(n \geq 2)$, for which chaos has been established either theoretically or numerically possess horseshoes and transversal homoclinic orbits. However, it is usually difficult to establish the existence of horseshoes for an arbitrary dynamical system. A theorem of Shilnikov provides sufficient conditions for the existence of horseshoes in the Poincare map of a three-dimensional, continuous-time system (Guckenheimer and Holmes, 1983; Arneodo, Coullet, and Tresser, 1981).

[5] For details see Arneodo, Coullet, and Tresser (1981, 1982), Glendinning and Sparrow (1984), Lorenz (1989), and de Vilder (1995).

[6] A geometrical description of the dynamical behaviour in these spiral-type attractors can be found in Berge et al. (1986).

[7] The calculated Lyapunov exponents for similar models, when the parameters are adequate selected, yield a positive and a negative exponent, in addition to zero exponent (see Lorentz, 1989 for details). 


\section{References}

Albu, L.-L. (1995): La modélisation de l'économie souterraine et des politiques fiscales, CEPREMAP - Paris, ACE - PHARE, August.

Ameodo, A., Coullet, P., and Tresser, C. (1981): "Possible New Strange Attractors with Spiral Structure", Communications in Mathematical Physics 79.

Arneodo, A., Coullet, P., and Tresser, C. (1982): "Oscillations with Chaotical Behavior: An Ilustration of a Theorem by Shilnikov", Journal of Statistics Physics 27. Wiley.

Berge, P., Pomeau, Y., and Vidal, C. (1986): Order within Chaos. New York:

Dăianu, D. and Albu, L.-L. (1996): Strain and the Inflation-Unemployment Relationship: A Conceptual and Empirical Investigation. Econometric Inference into the Macroeconomic Dynamics East European Economies, University of Leicester, UK. Research Memorandum, No. 96/15.

Fischer, E.O. and Jammernegg, W. (1986): "Empirical Investigation of a Catastrophe Extension of the Phillips-curve", Review of Economics and Statistics.

Friedman, M. (1968): "The Role of Monetary Policy", American Economic Review 58.

Gandolfo, G. (1983): Economic Dynamics: Methods and Models. 2nd ed., Amsterdam: North-Holland.

Glendinning, P. and Sparrow, C. (1984): "Local and Global Behavior near Homoclinic Orbits", Journal of Statistical Physics 35.

Goodwin, R. (1967): "A Growth Cycle". Socialism, Capitalism and Economic Growth. C.H. Feinstein (ed.). Cambridge University Press.

Guckenheimer, J. and Holmes, P. (1983): Nonlinear Oscillations, Dynamical Systems, and Bifurcations of Vector Field. New York-Berlin-Heidelberg: Springer.

Lipsey, R.G (1960): "The Relation between Unemployment and the Rate of Change of Money Wage Rates in the United Kingdom, 1862-1957: A Further Analysis", Economica 27.

Lorenz, H.W. (1989): Nonlinear dynamical economics and chaotic motion. Lecture Notes in Economic and Mathematical Systems 334. Springer-Verlag.

Mandelbrot, Benoît (1972): "Statistical Methodology for Non-Periodic Cycles:

From the Covariance to R/S Analysis", Annals of Economic and Social Measurement 1.

May, R.M. (1976): "Simple Mathematical Models With Very Complicated Dynamics", Nature 261.

Metzler, L.A. (1941): "The Nature and Stability of Inventory Cycles", Review of Economic Studies 23.

Mundell, R.A. (1990): "Debts and Deficits in Alternative Macroeconomic Models", Revista di Politica Economica, VII-VIII, Roma.

Phillips, A.W. (1958): "The Relation between Unemployment and the Rate of Change of Money Wage Rates in the United Kingdom, 1861-1957", Economica 25.

Pohjola, M.T. (1981): "Stable, Cyclic and Chaotic Growth: the Dynamics of a Discrete Time Version of Goodwin's Growth Cycle Model”, Zeitschrift fur Nationalekonomie 41.

Santeremo, A.M. and Seater, J.L. (1978): "The Inflation Unemployment Trade-Off: A Critique of the Literature", Journal of Economic Literature 16.

Soliman, A.S. (1996): "Transition from Stable Equilibrium Points to Periodic Cycles to Chaos in a Phillips Curve System", Journal of Macroeconomics, 18.

Woodcock, A.E.R. and Davis, M. (1979): Catastrophe Theory. London: Penguin. 
Annex 1a
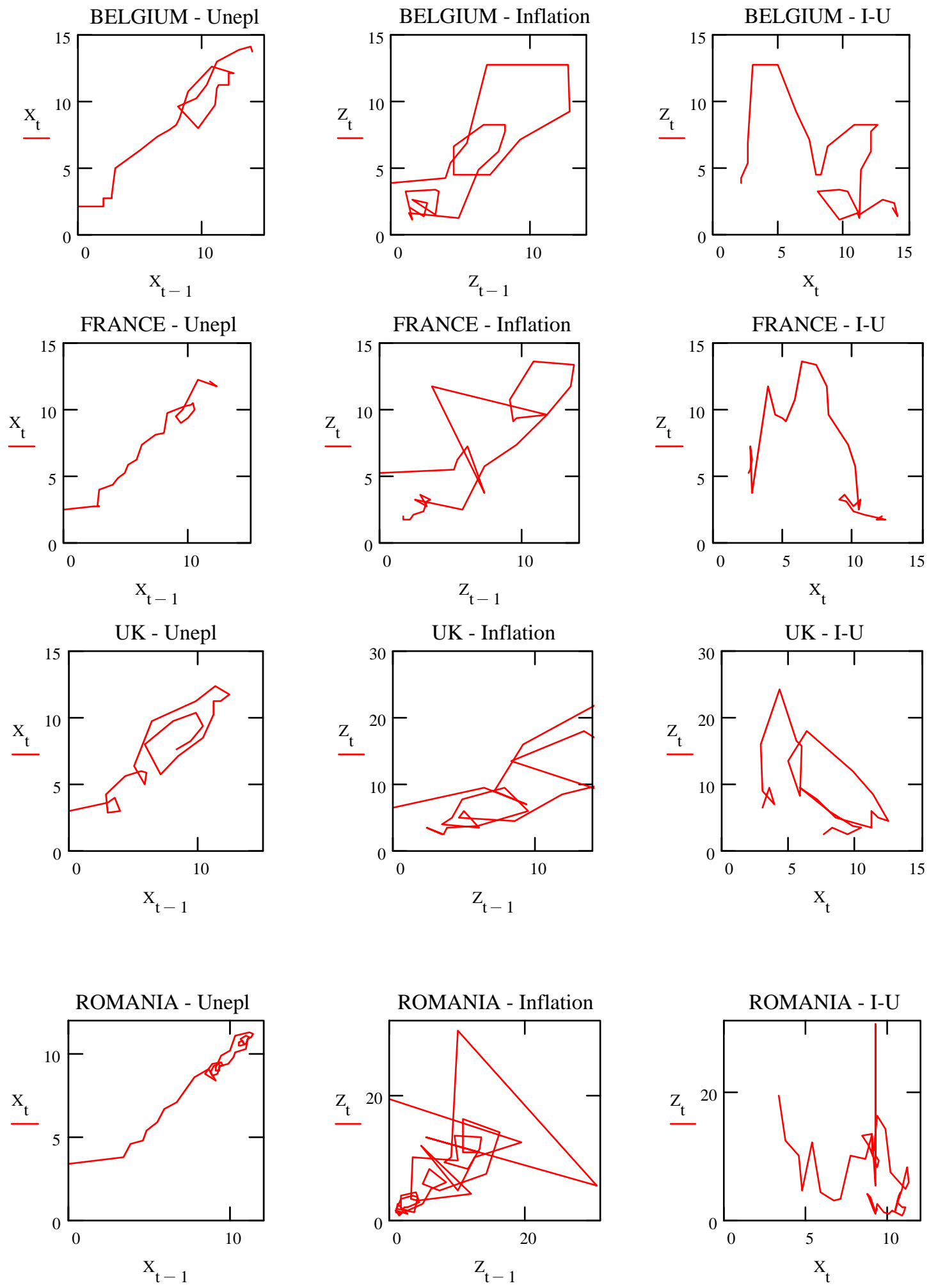
Annex 1b
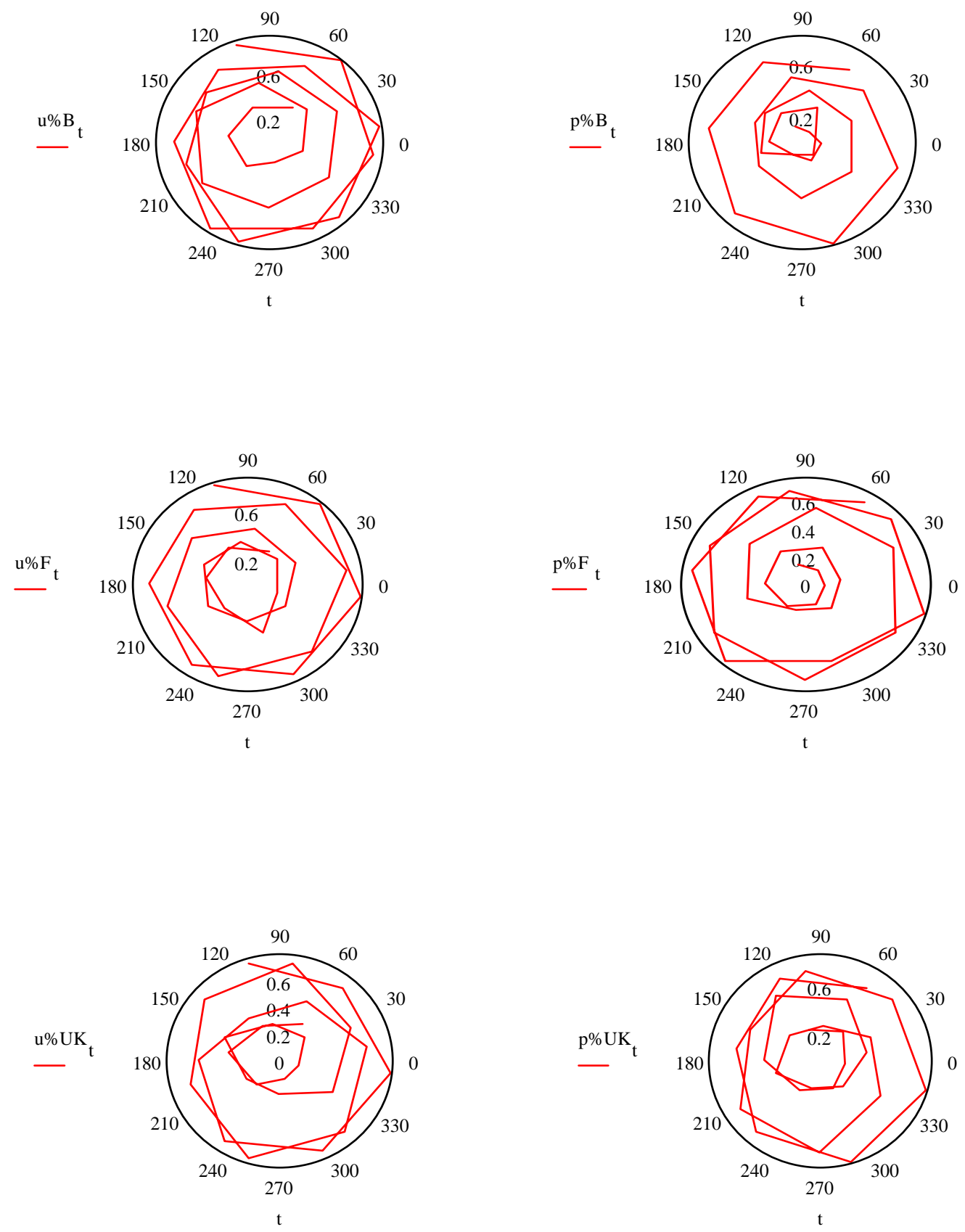
(continued)
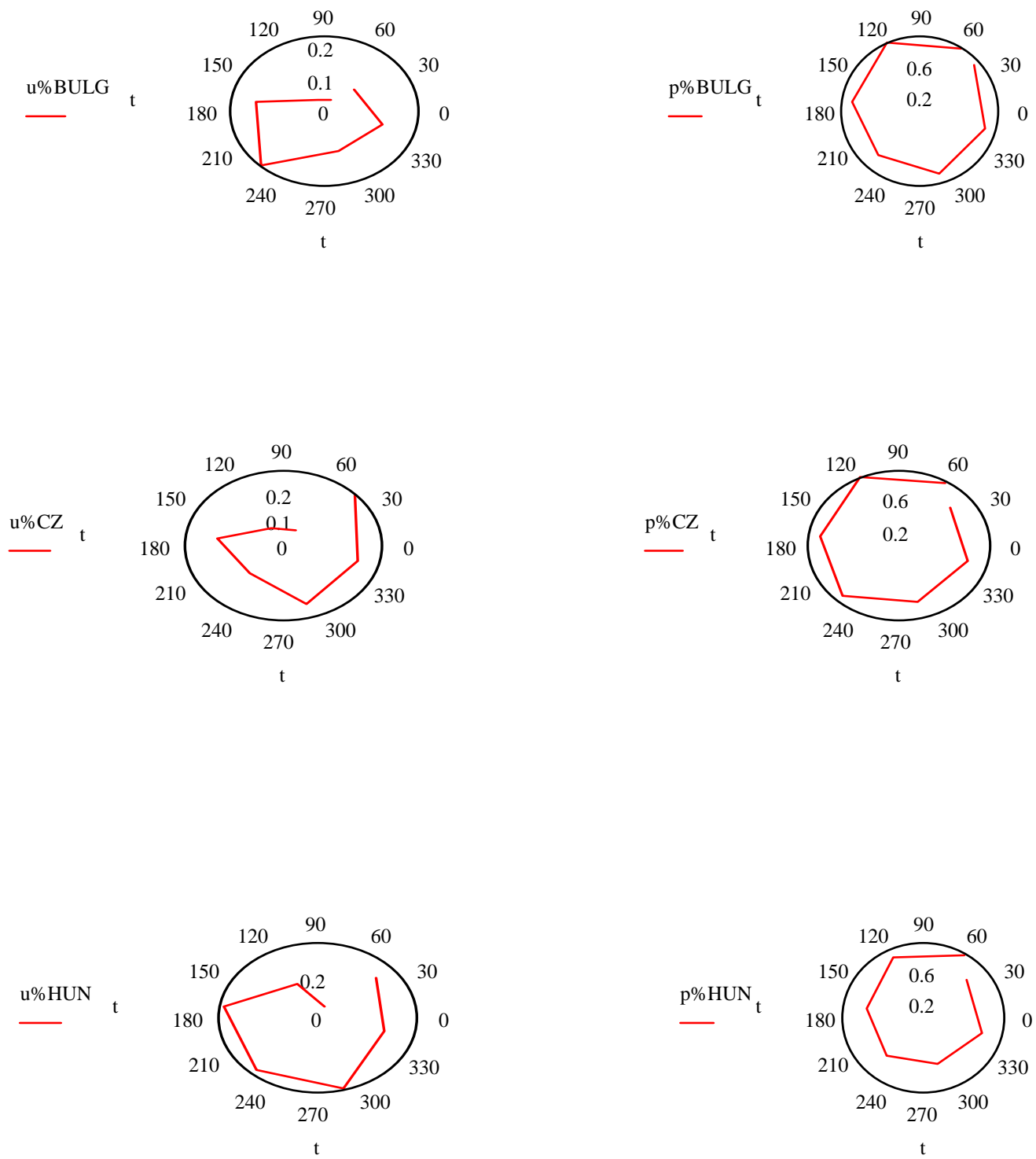
(continued)
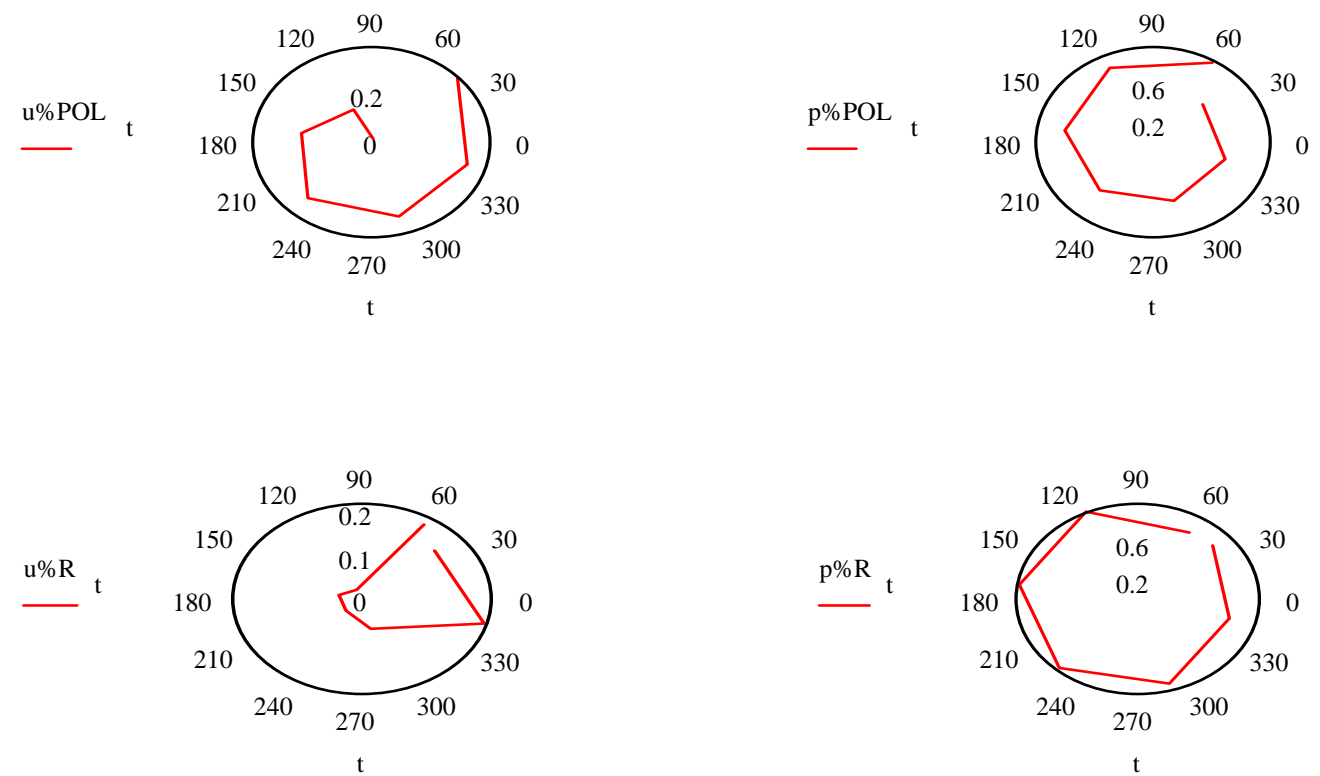
Annex 2a
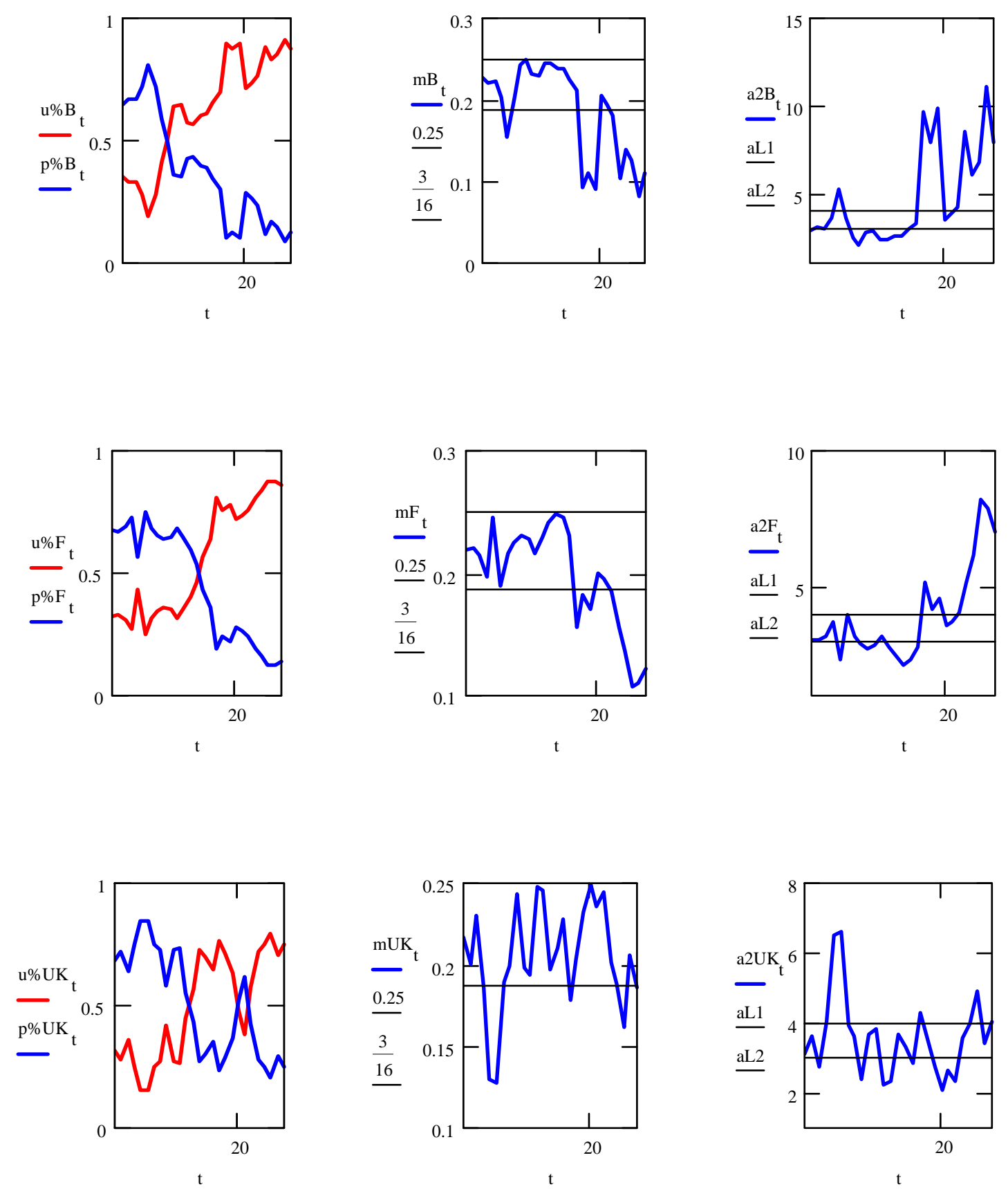
Annex 2b
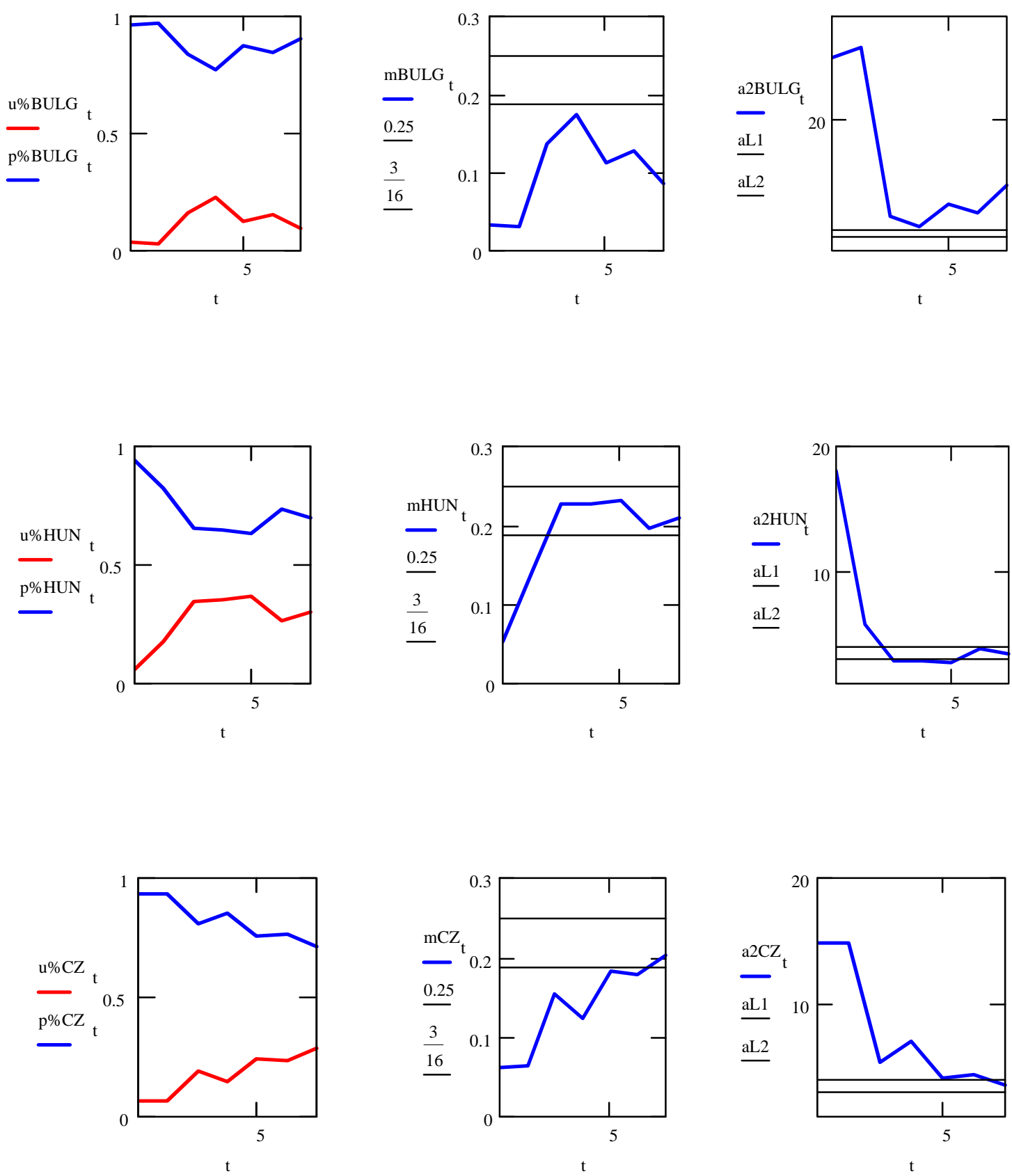
(continued)
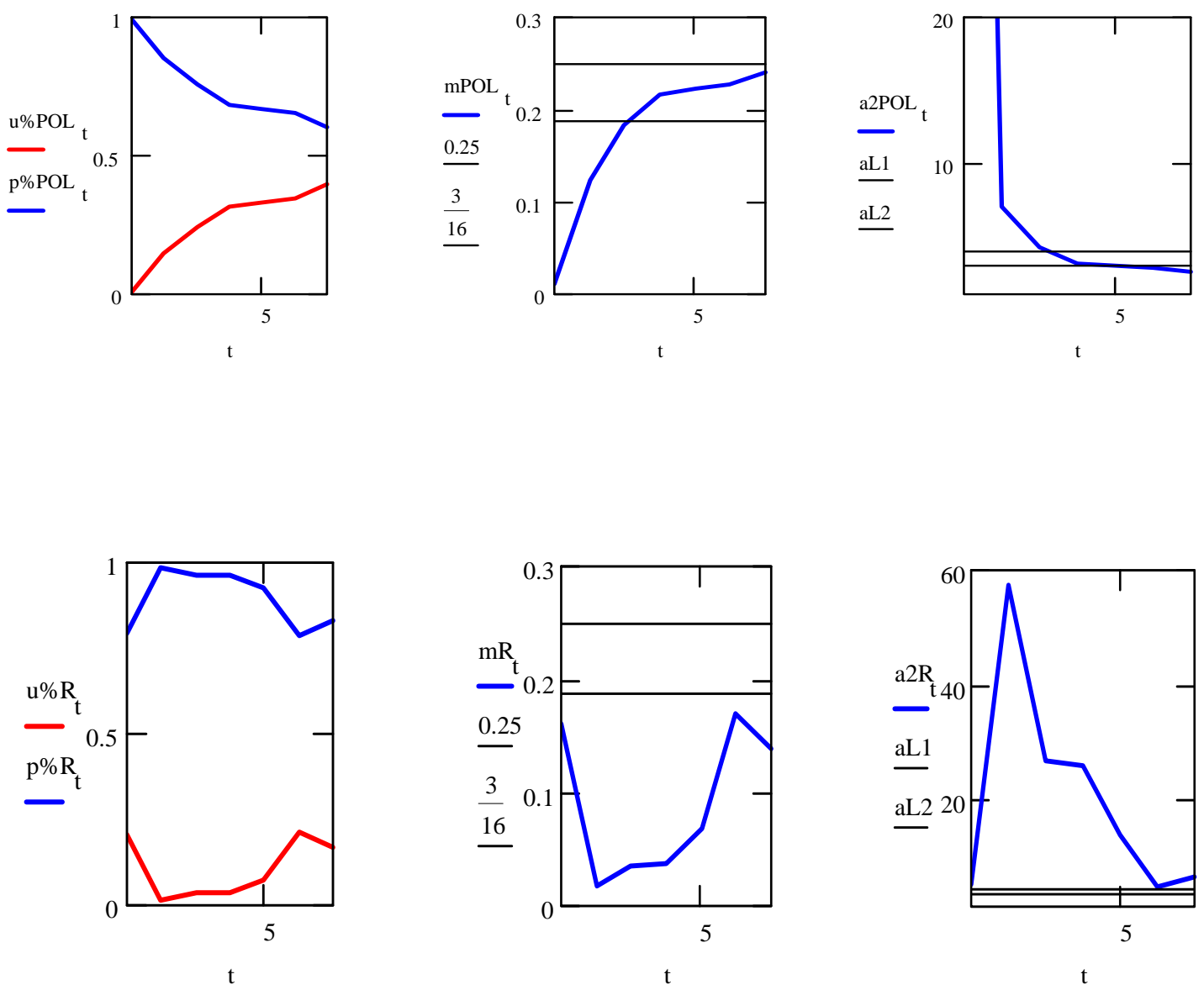
Annex 3

By applying the methodology on data relating to the selected Western countries resulted the estimate values of $\mathrm{H}$ for the period 1970-1995 which are presented in the following table:

\begin{tabular}{lll}
\hline & Hdoup & Hdo \\
\hline Belgium & 0.861 & 0.841 \\
Denmark & 0.889 & 0.897 \\
France & 0.901 & 0.906 \\
Germany & 0.773 & 0.677 \\
Greece & 0.823 & 0.812 \\
Italy & 0.943 & 0.938 \\
Ireland & 0.739 & 0.747 \\
Netherlands & 0.792 & 0.754 \\
Portugal & 0.978 & 0.966 \\
Spain & 0.909 & 0.907 \\
United Kingdom & 0.885 & 0.856 \\
\hline
\end{tabular}

As a comparison, but less relevance because of the monthly data, for Romania the calculated value of Hdoup was about 0.895 for a period of about of 3 year 1993-1996 (the monthly inflation rate was replaced by one yearly equivalent rate). 
Annex 4a

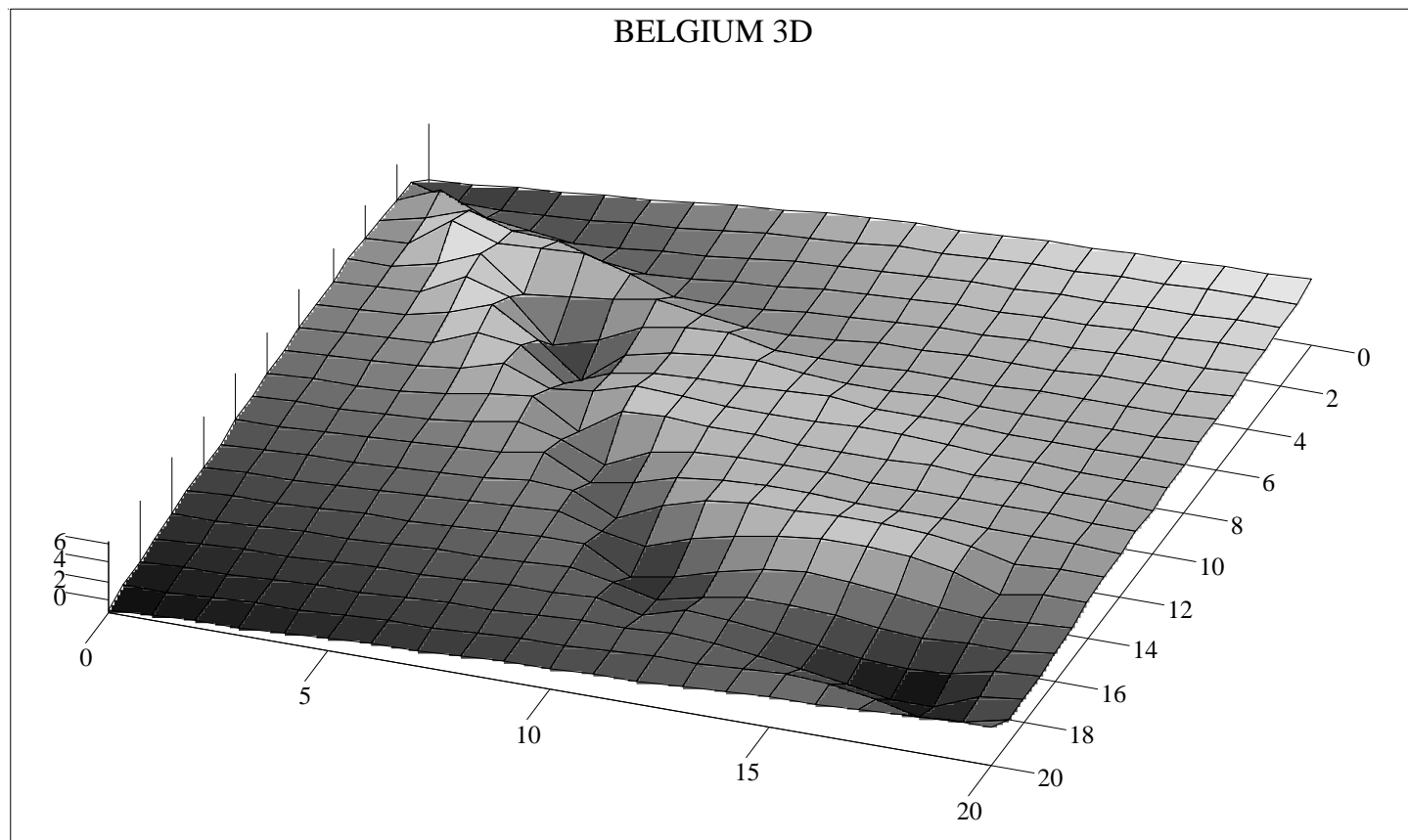

$\mathrm{x}$, ylc , y

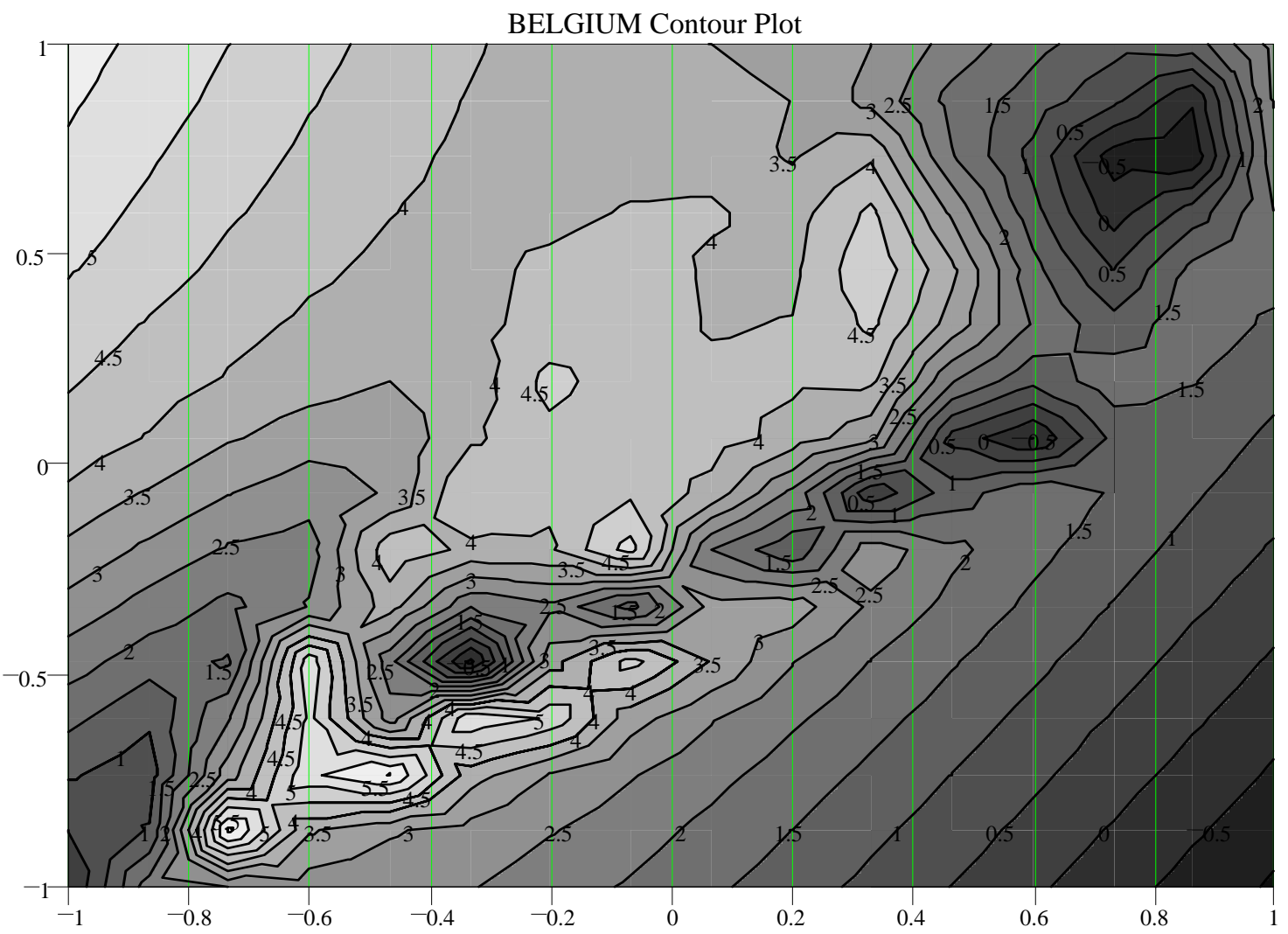

$\mathrm{x}$, ylc , y 
Annex 4b

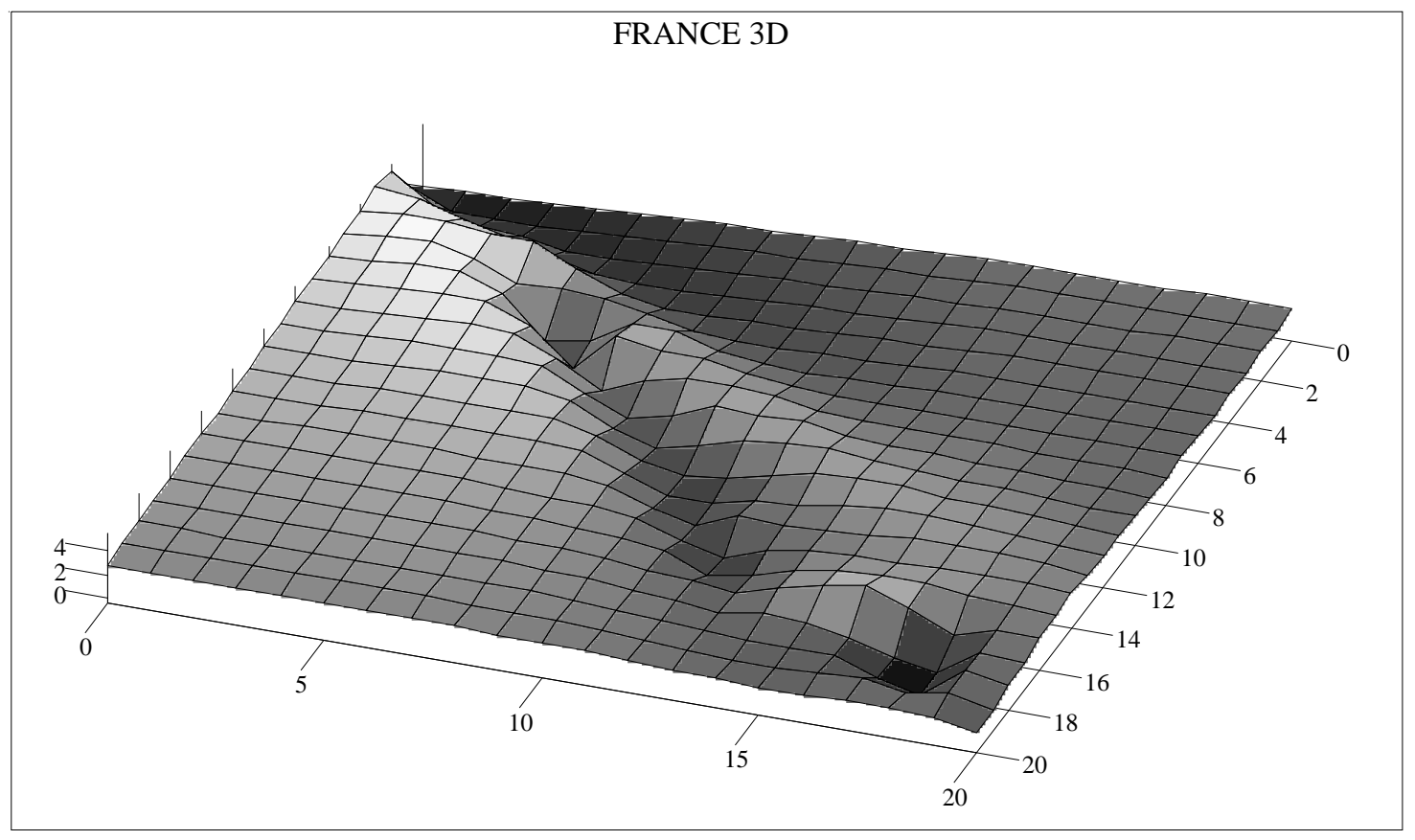

$\mathrm{x}$, ylc , y

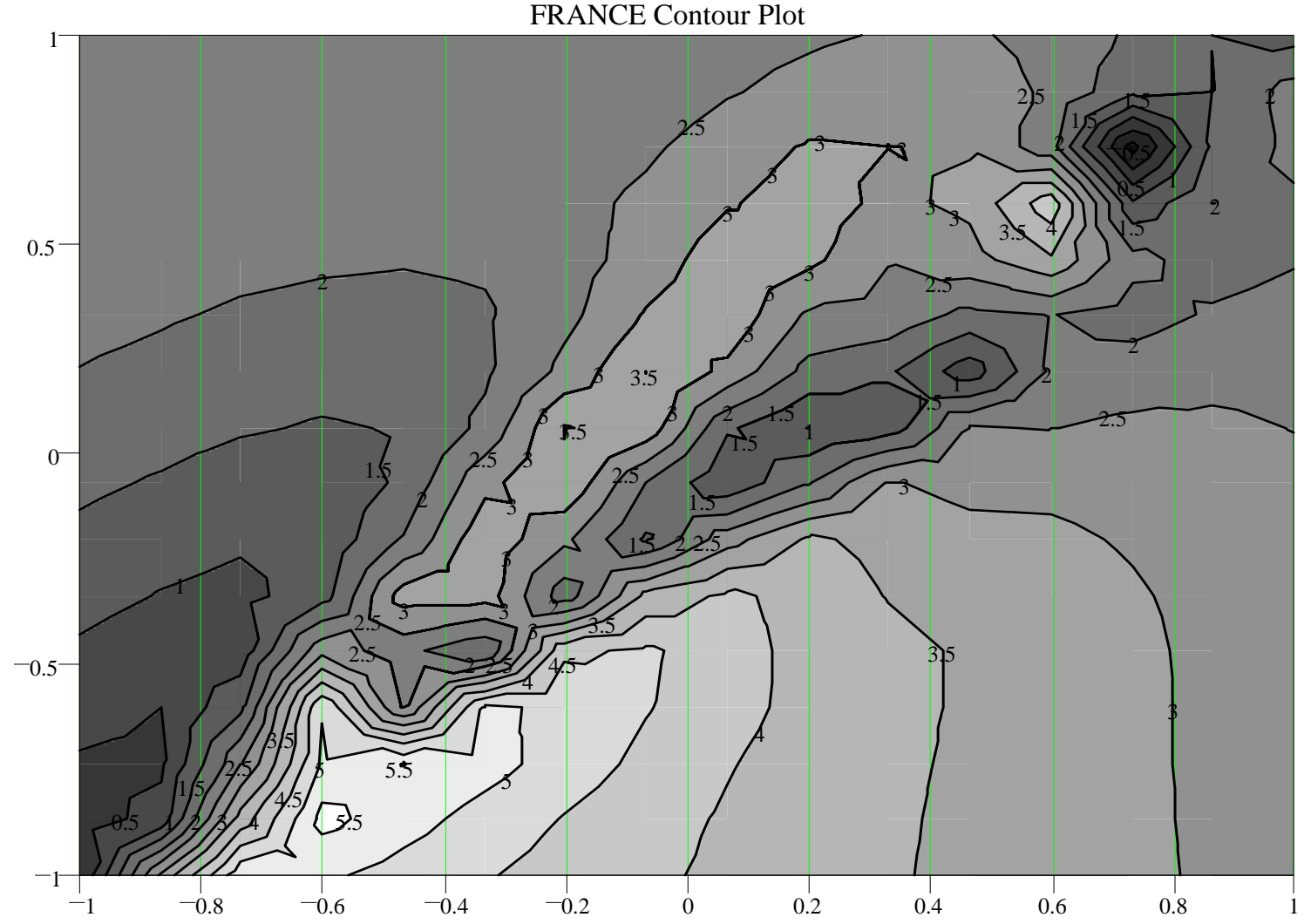

$\mathrm{x}$, ylc , y 
Annex 4c

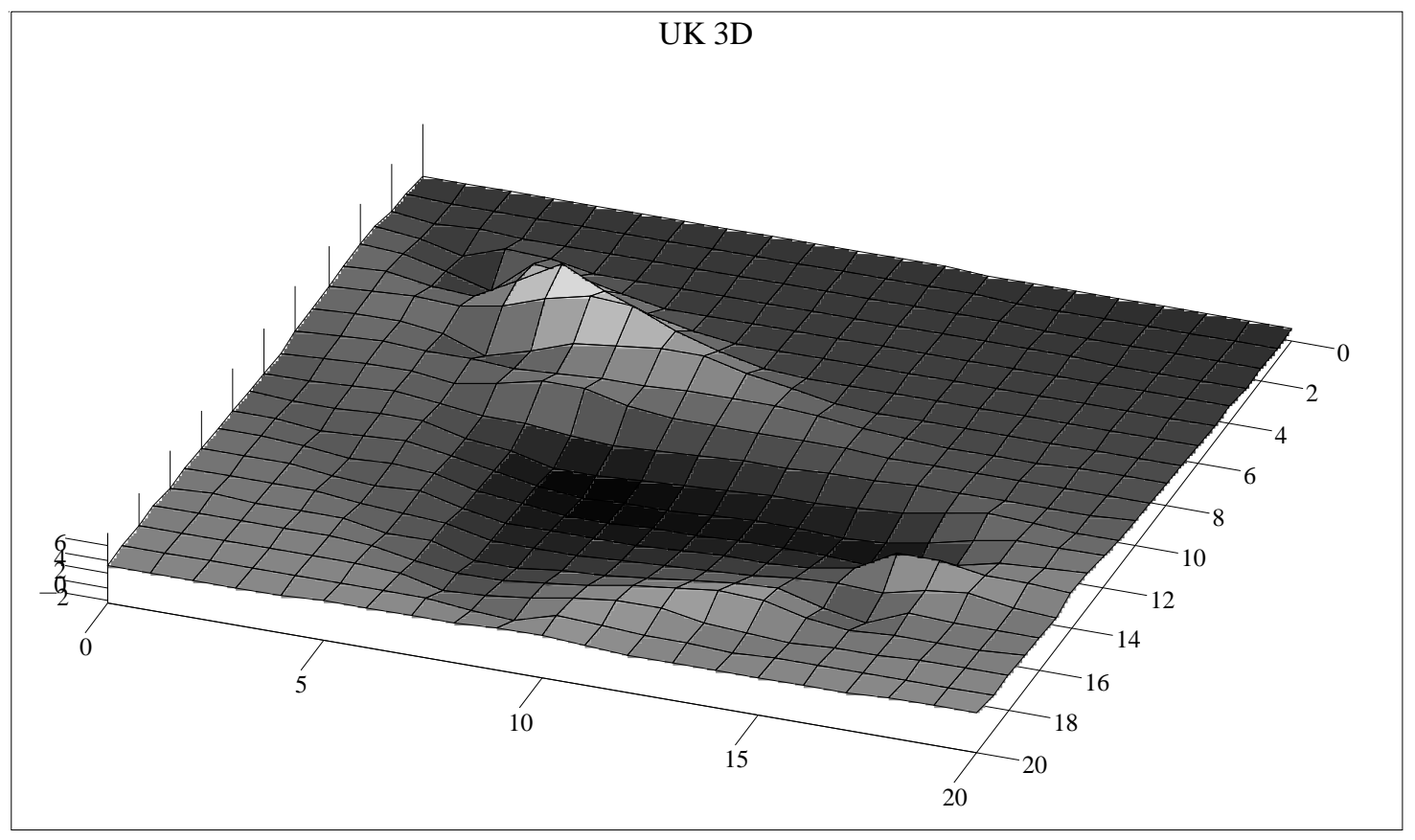

$\mathrm{x}$, ylc , y

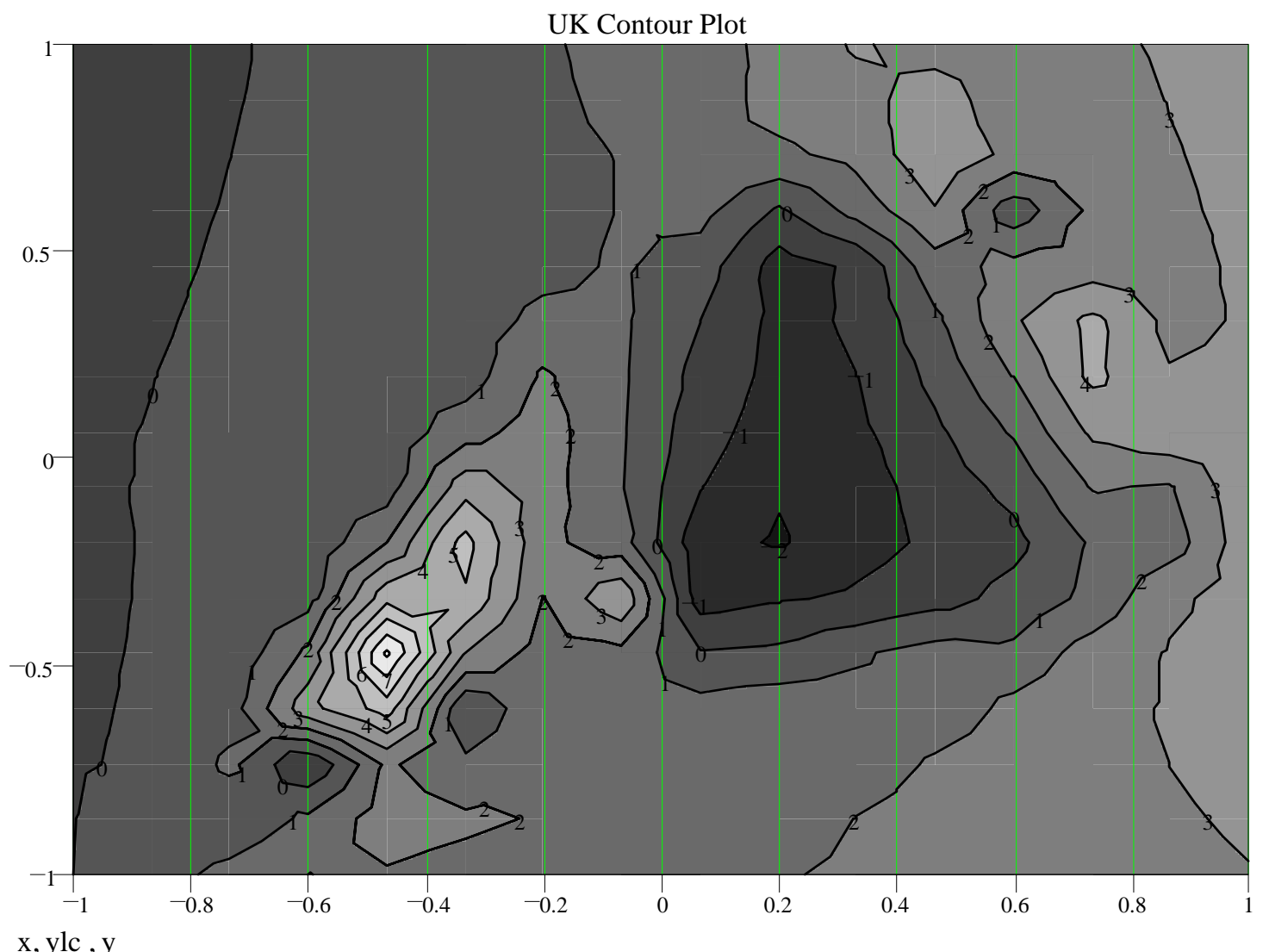

$\mathrm{x}$, ylc , $\mathrm{y}$ 
Annex 5
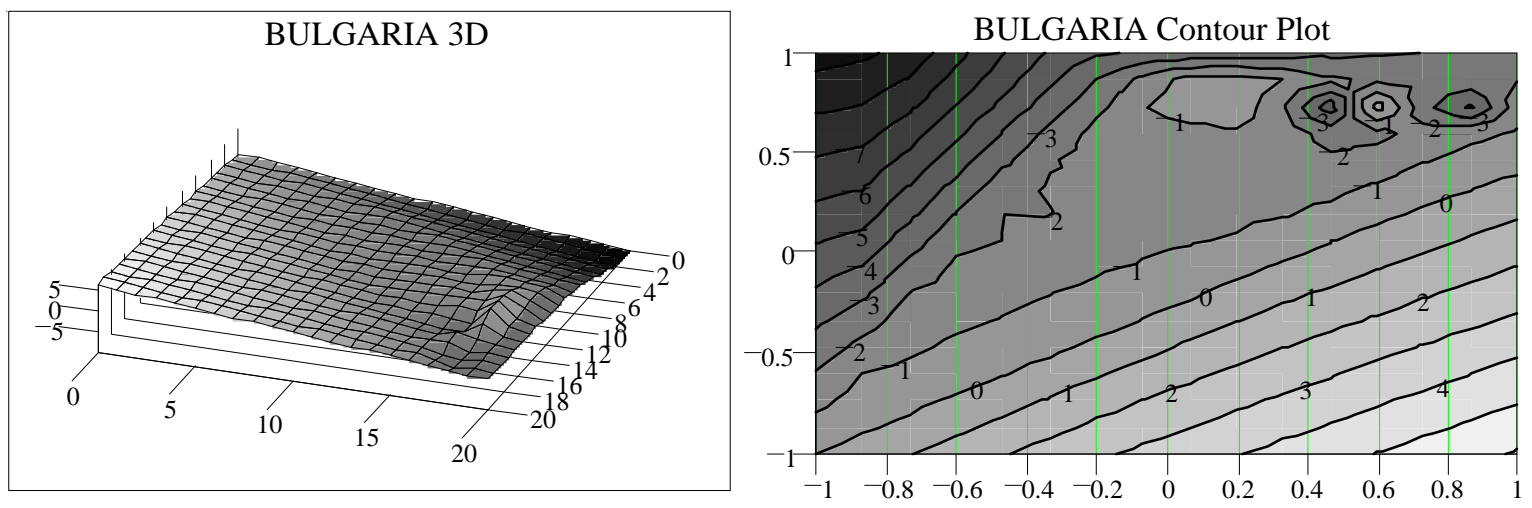

$\mathrm{x}$, ylc, y

$\mathrm{x}$, ylc , y
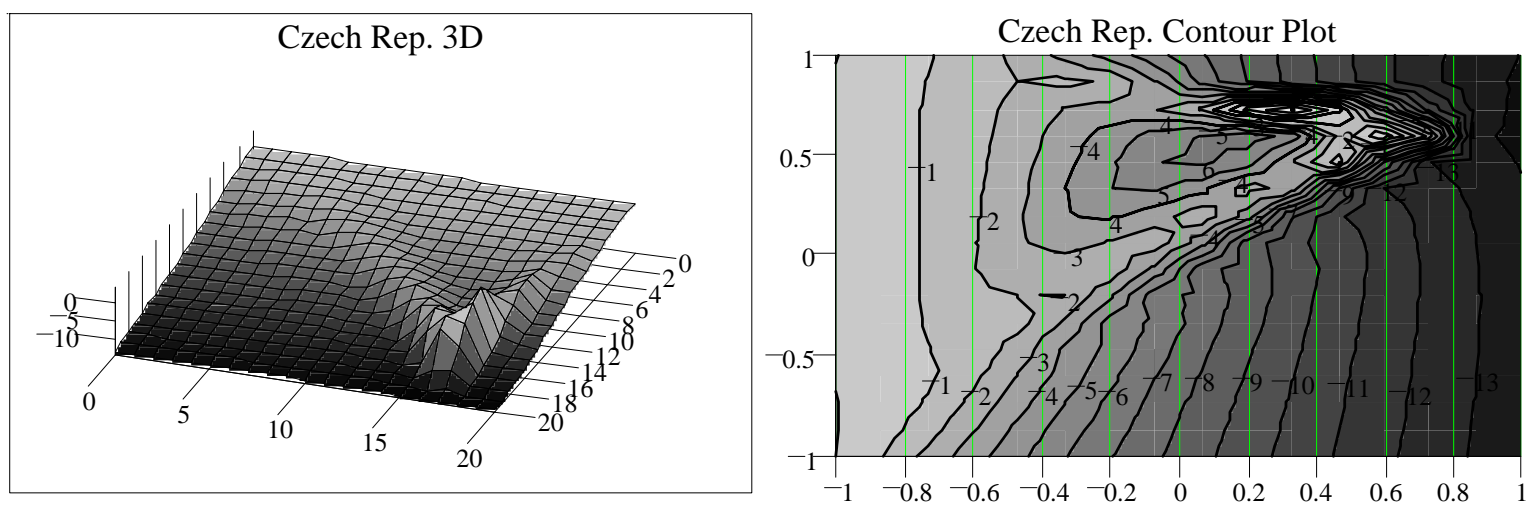

$\mathrm{x}$, ylc, $y$

$\mathrm{x}$, ylc, $\mathrm{y}$
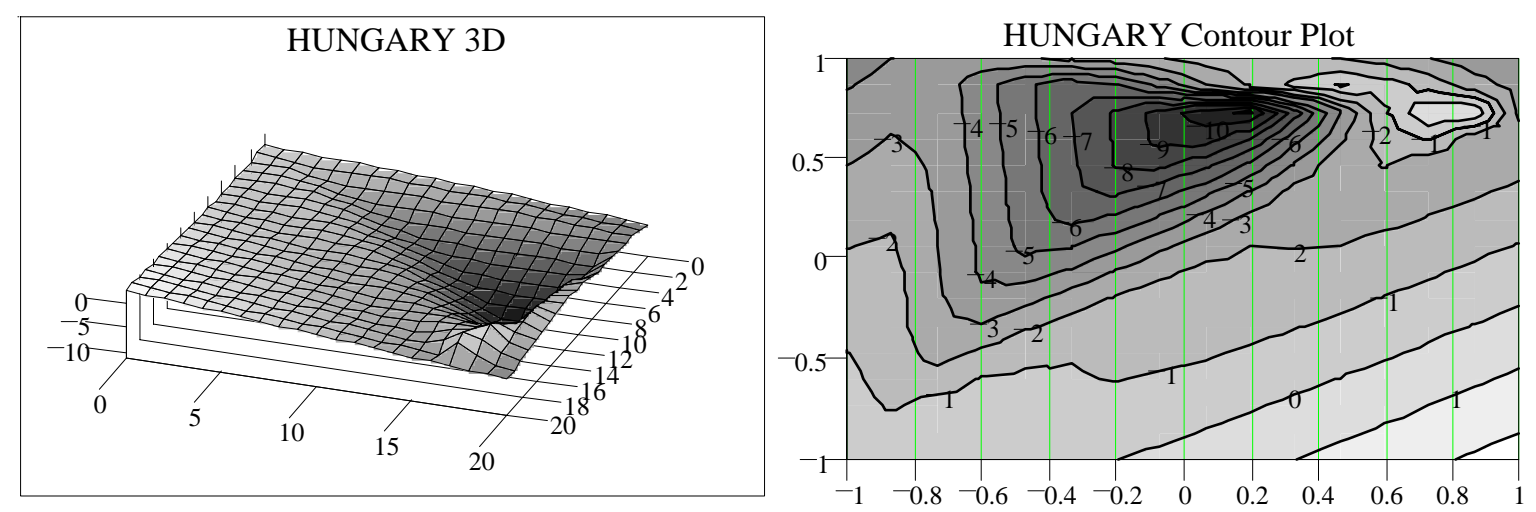

$\mathrm{x}, \mathrm{ylc}, \mathrm{y}$

$\mathrm{x}$, ylc, y 


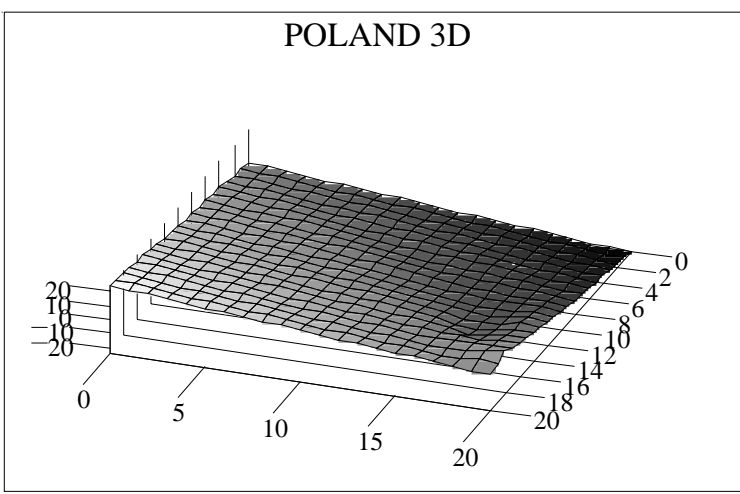

x, ylc, y

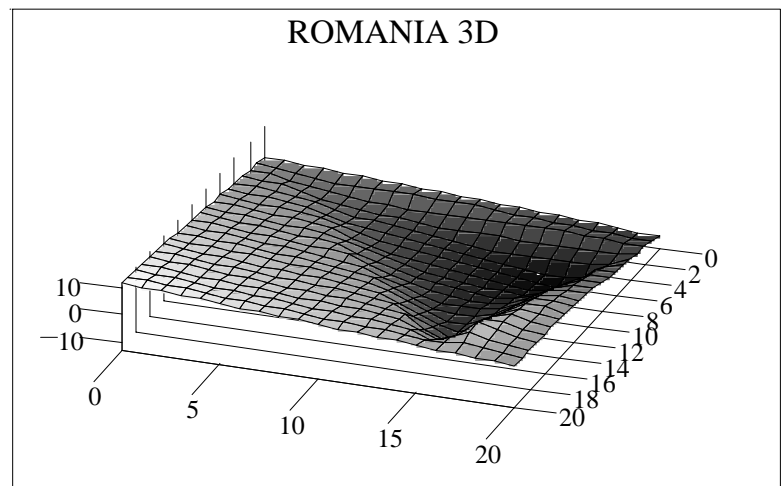

$\mathrm{x}$, ylc , y

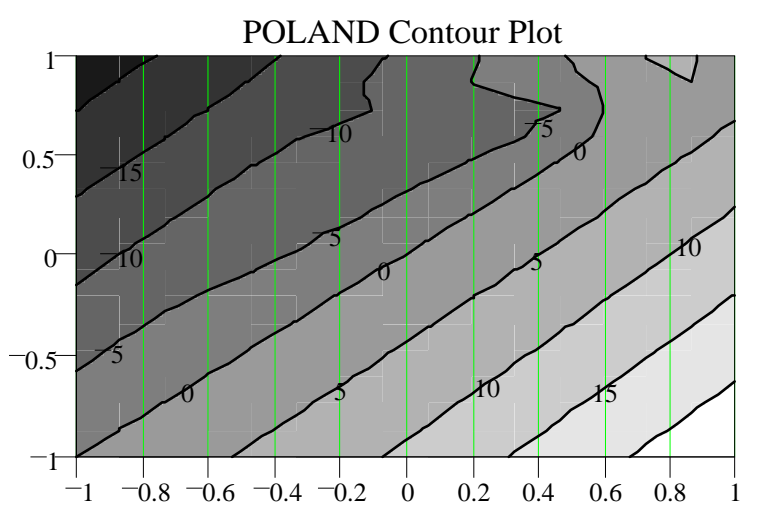

$\mathrm{x}$, ylc, y

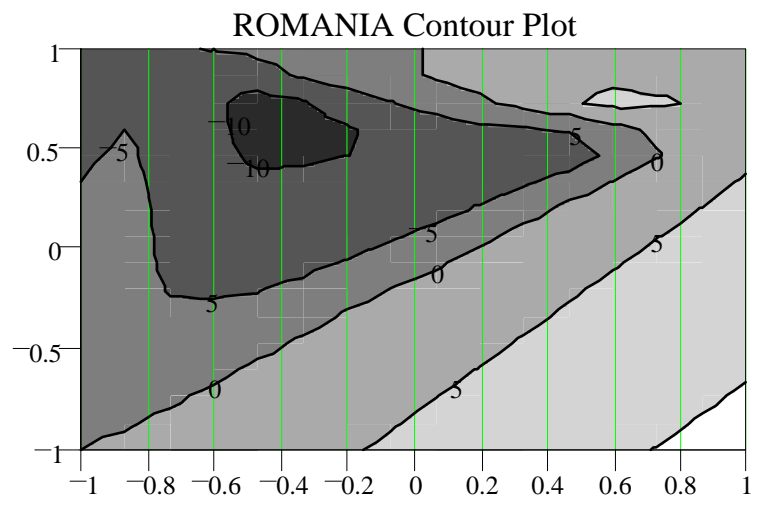

$\mathrm{x}$, ylc, $\mathrm{y}$ 
Annex 6a

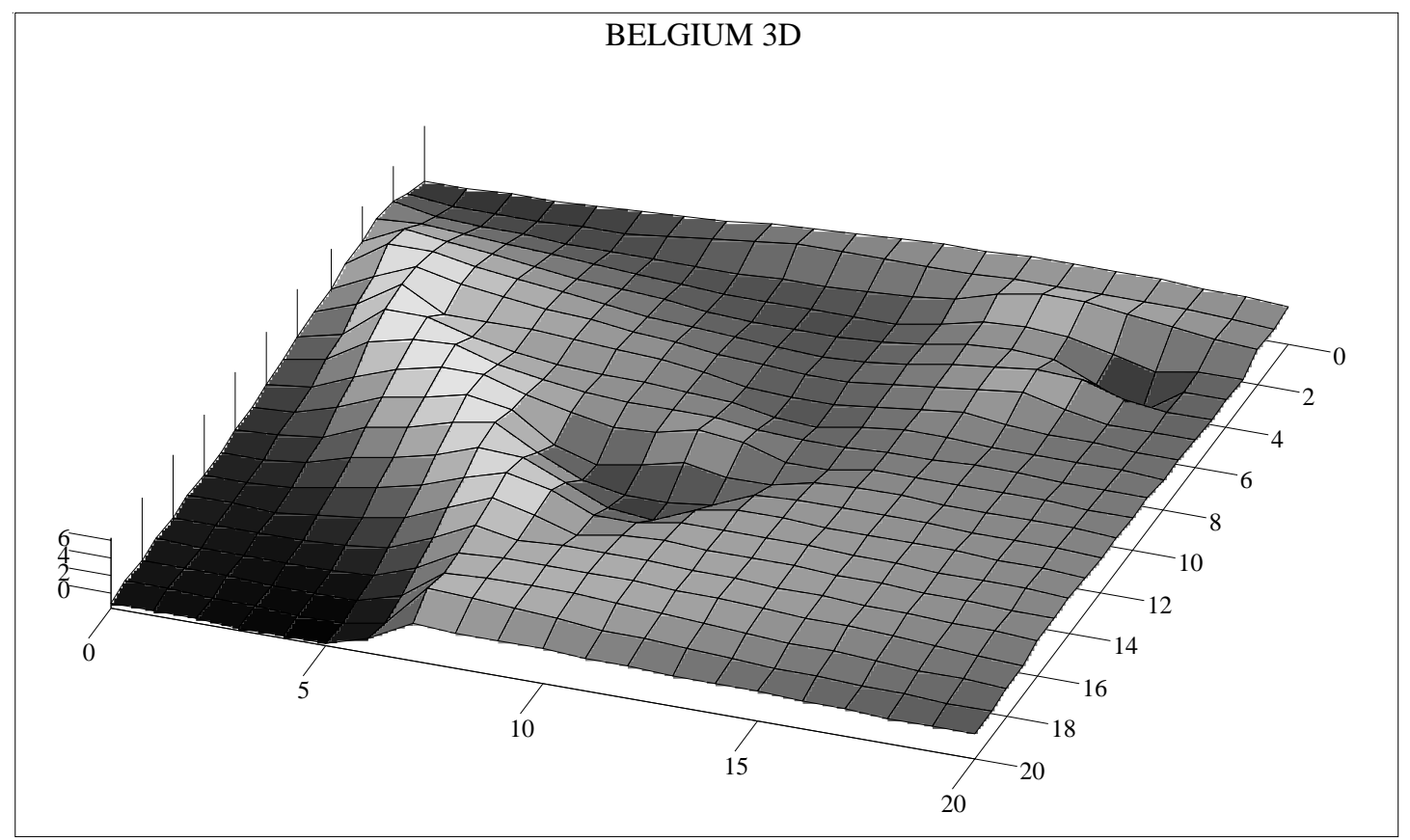

z, ylc , y

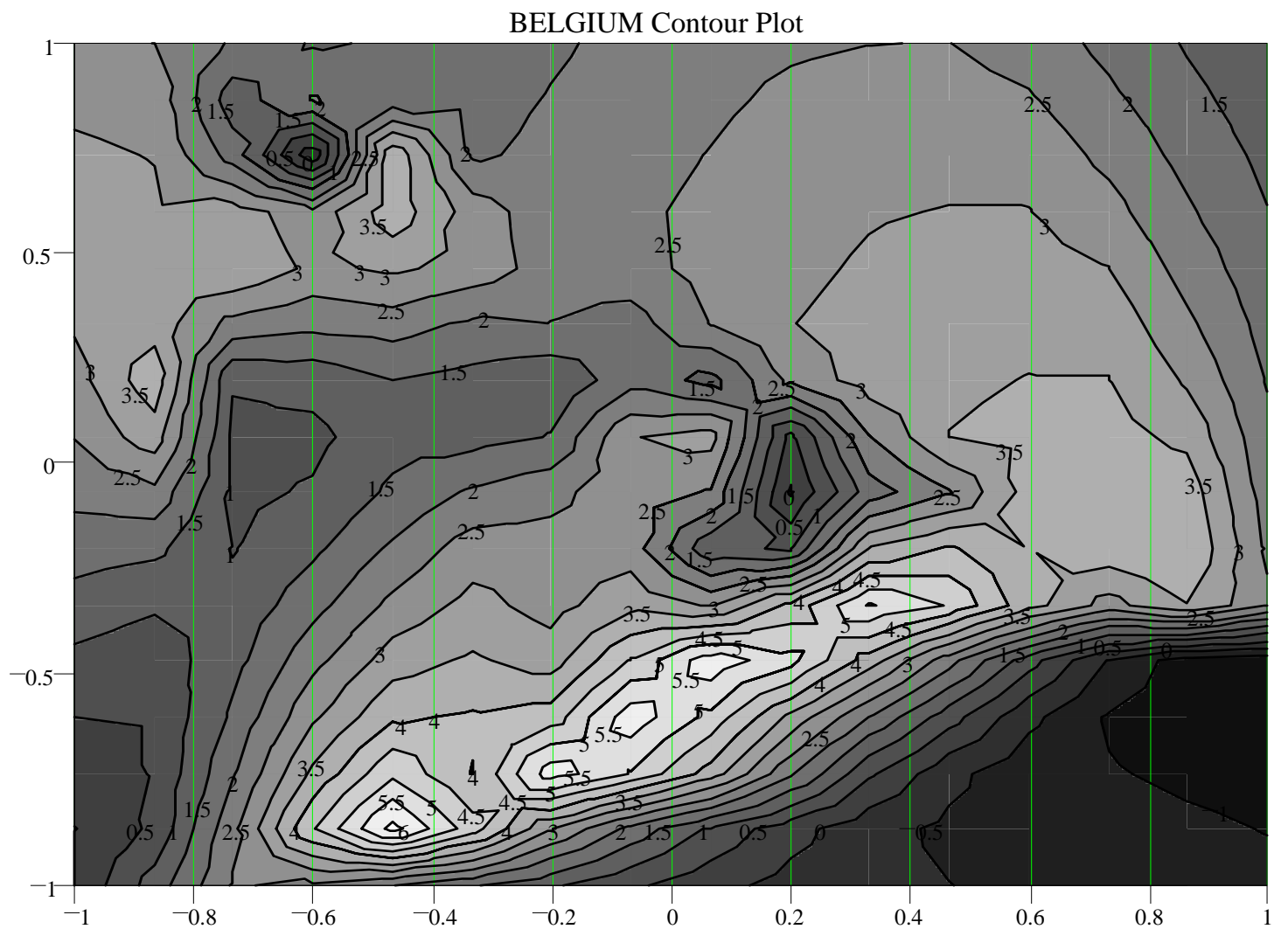

z, ylc, y 
Annex 6b

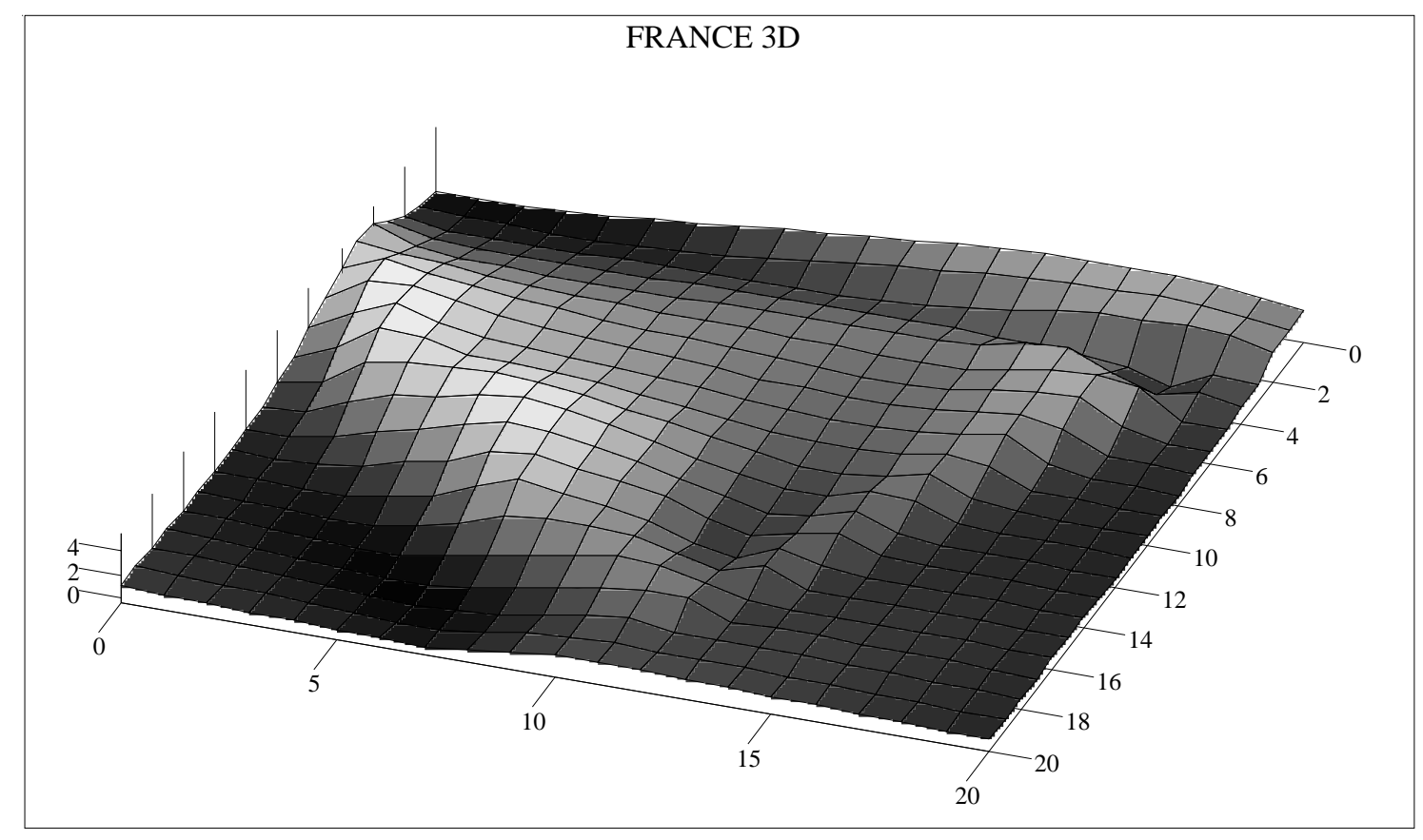

z, ylc, y

FRANCE Contour Plot

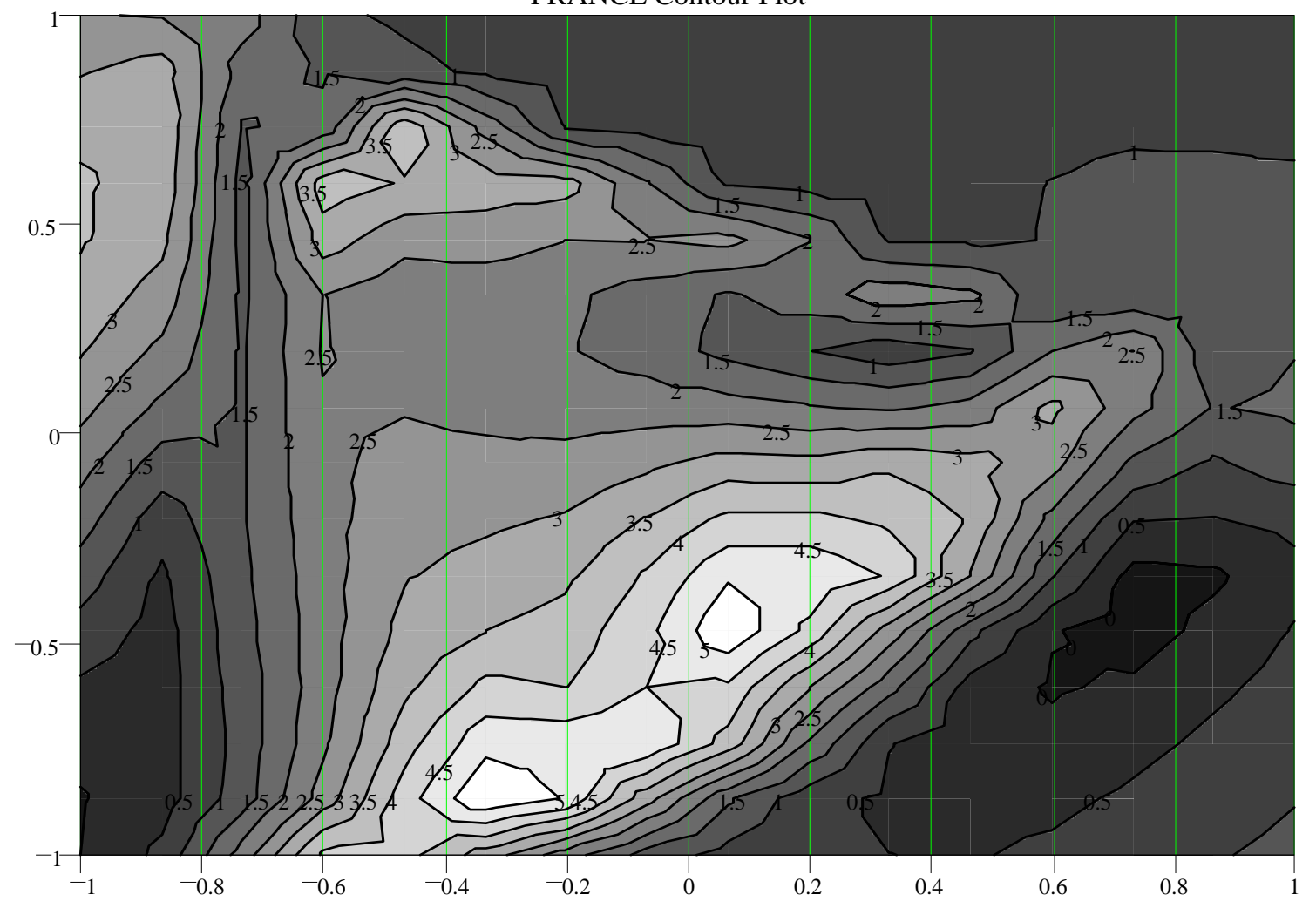

z, ylc, y 
Annex 6c

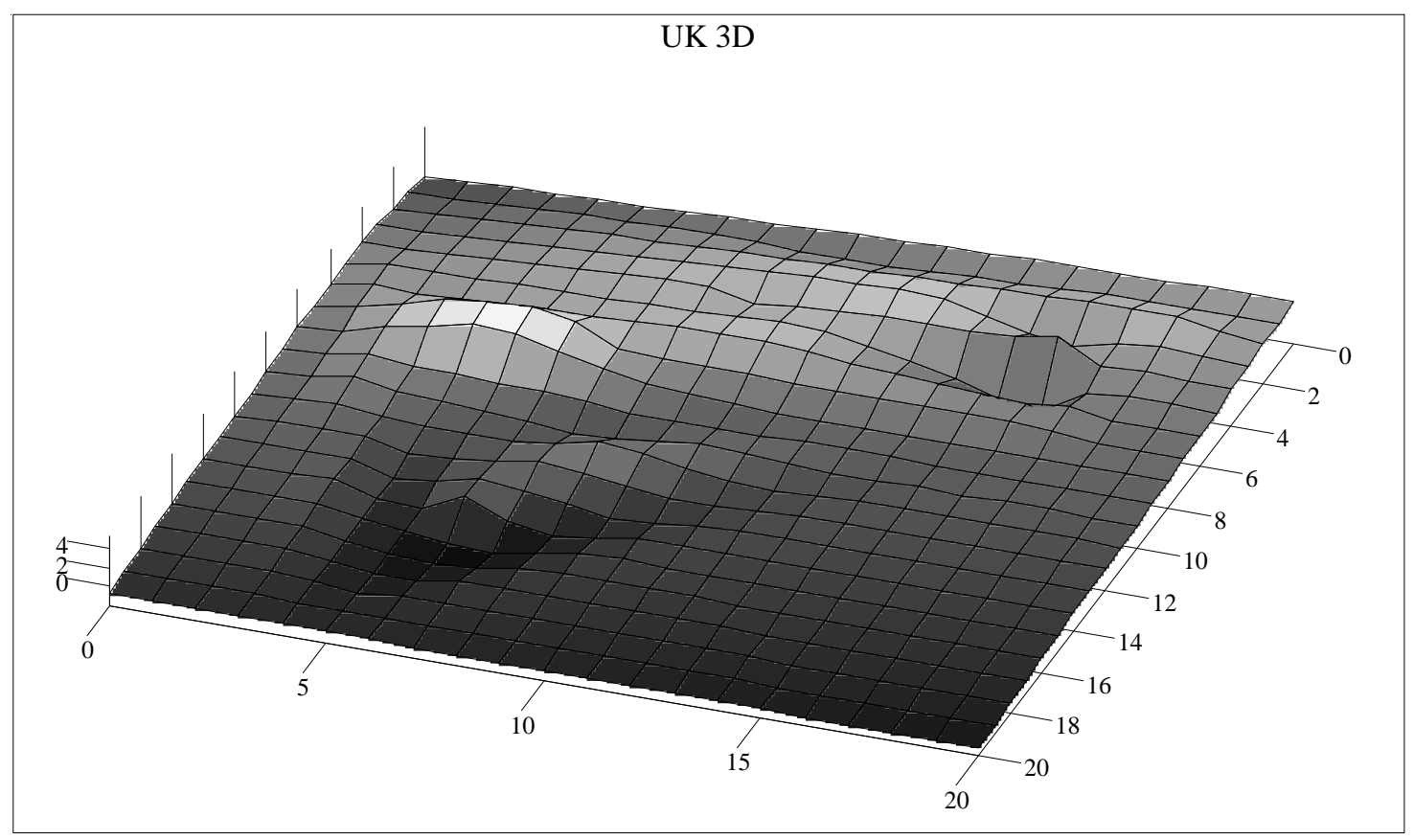

z, ylc , y

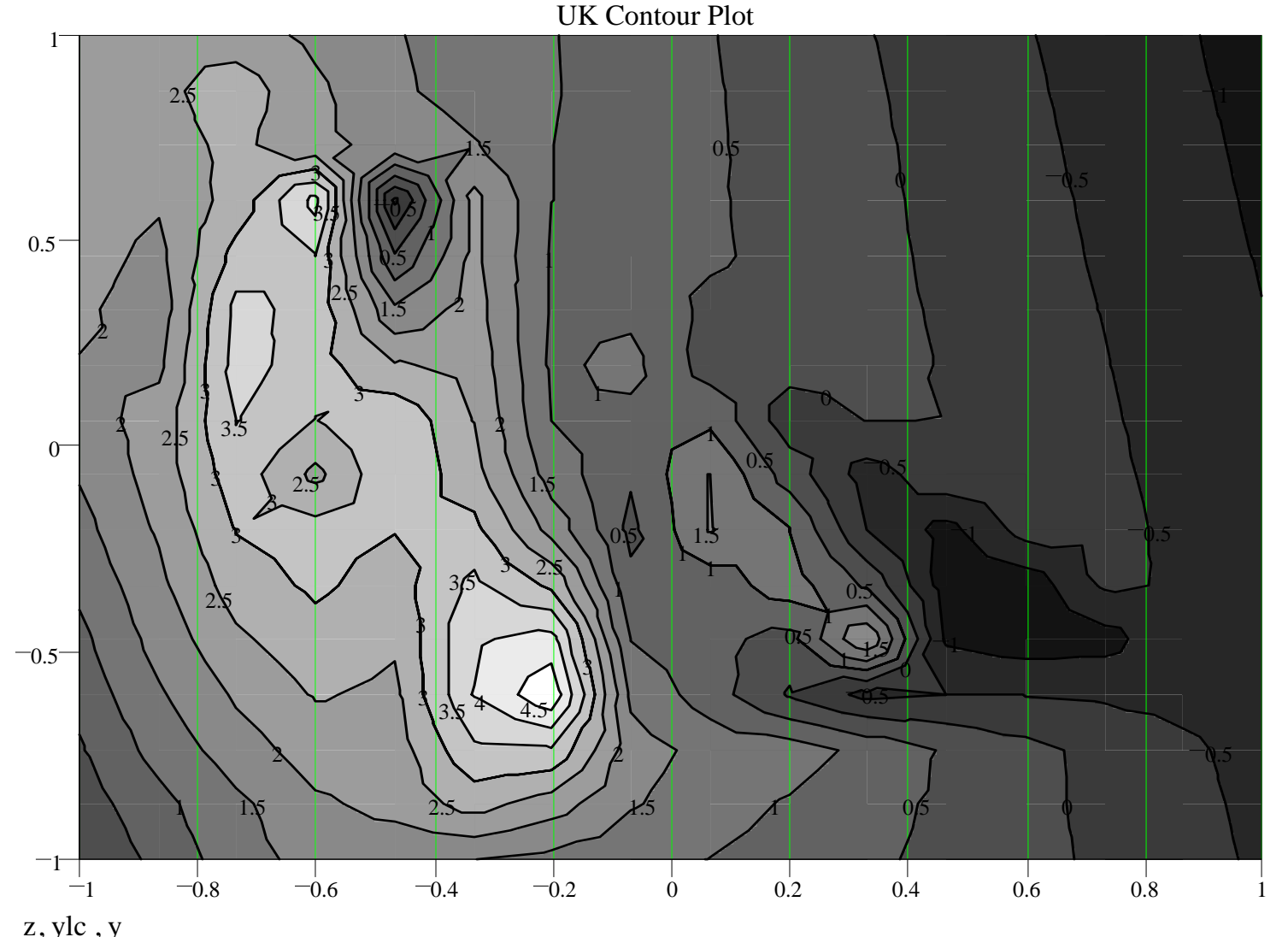


Annex 7
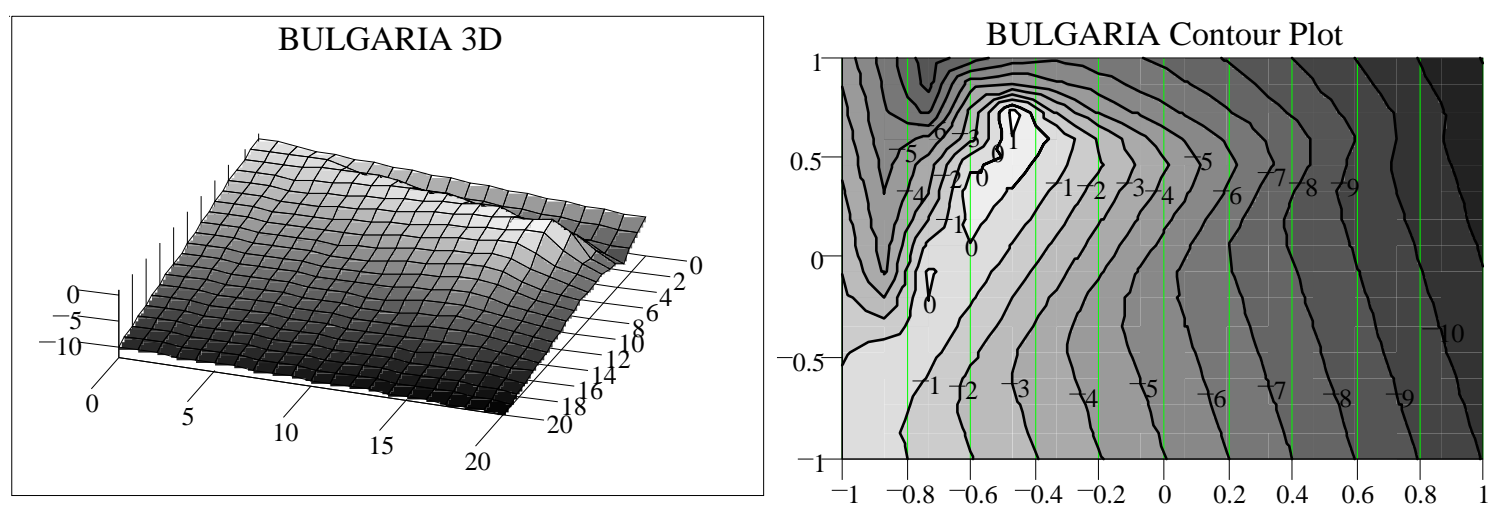

z, ylc, y

z, ylc, y
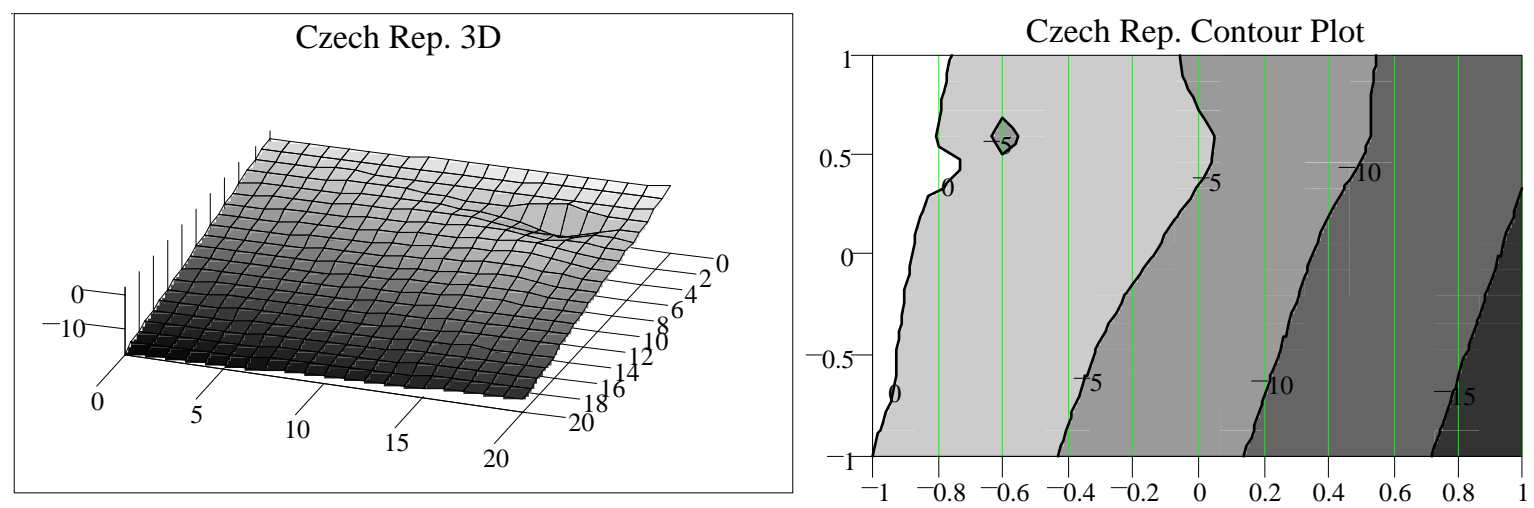

z, ylc, y

z, ylc, y
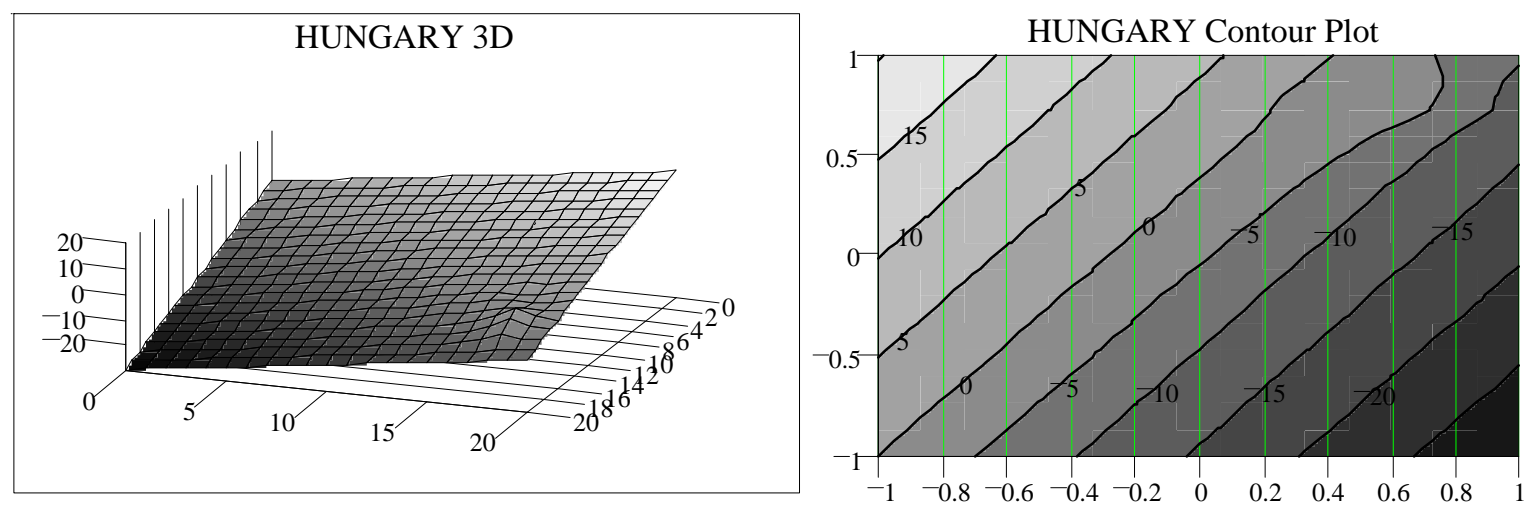

z, ylc, y z, ylc, y 
(continued)
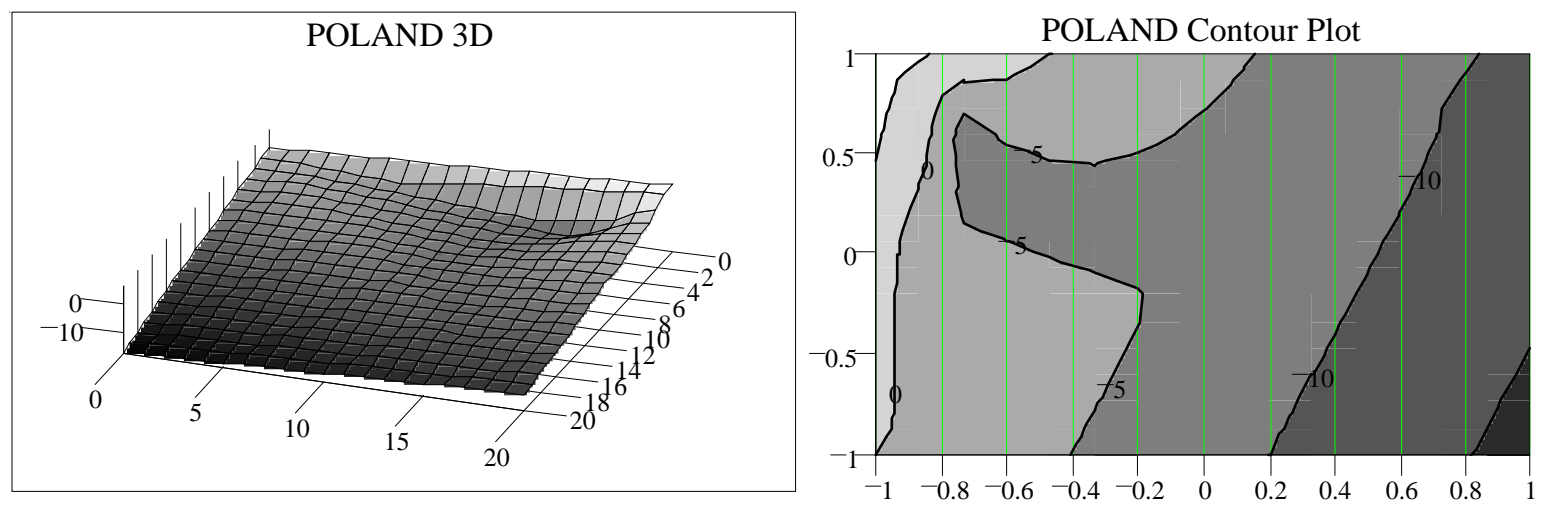

z, ylc , y

z, ylc, y

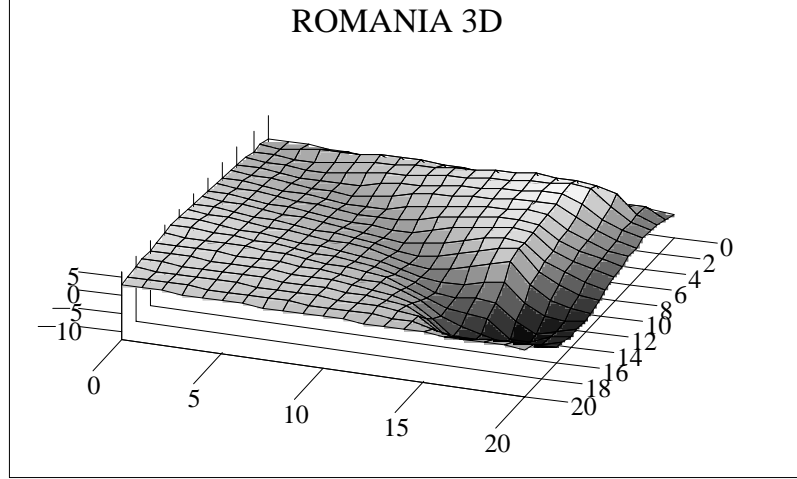

z, ylc , y

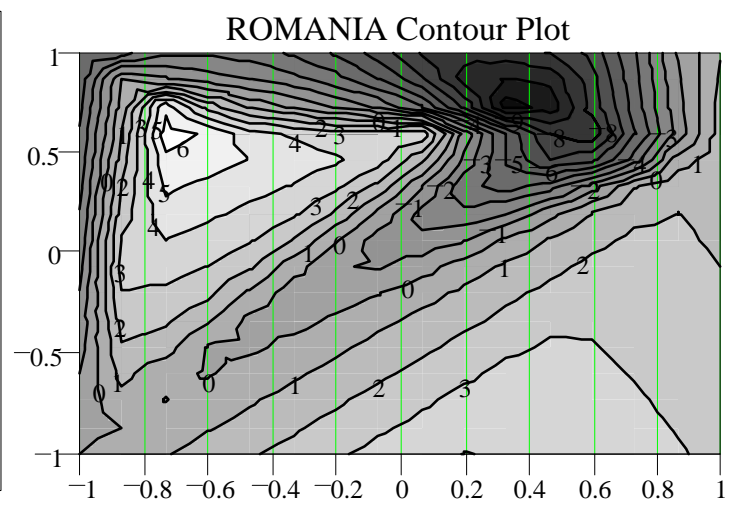

z, ylc, y 


\section{Some theoretical considerations on the local bifurcations in continuous-time dynamical systems}

In this section we consider useful to present briefly the general considerations on the local bifurcations in continuous-time dynamical systems, in the sense that only the behaviour of a system in the neighbourhood of a single equilibrium point is affected.

Consider the ordinary differential equation

$\mathrm{dx} / \mathrm{dt}=f(\mathbf{x}, \mu), \quad \mathrm{x} \in \mathbf{R}, \mu \in \mathbf{R}$,

with $\mu$ as a parameter. Assume that (A8.1), for $\mu=\mu_{0}$, has an equilibrium point $\left(\mathrm{x}^{*}, \mu_{0}\right)$ such that $0=f\left(\mathrm{x}^{*}, \mu_{0}\right)$. The eigenvalue of $(\mathrm{A} 8.1)$ is given by $\lambda=\partial f(\mathrm{x}, \mu) / \partial \mathrm{x}$, and it is well-known that the equilibrium point is locally asymptotically stable as long as $\lambda<0$ at $\left(x^{*}, \mu_{0}\right)$. Assume that at $\left(x^{*}, \mu_{0}\right)$ the eigenvalue is equal to zero. It follows from the implicit function theorem that the equilibria of (A8.1) for values of $\mu$ different from $\mu_{0}$ can be expressed as a smooth function $x=x(\mu)$. The function $x(\mu)$ is called a branch of equilibria. If at $\left(x^{*}, \mu_{0}\right)$ several branches of equilibria come together, the point $\left(x^{*}, \mu_{0}\right)$ is said to be a bifurcation point. The presentation of branches of equilibria in $(x-\mu)$ - space is called a bifurcation diagram. The value of $\mu$ at which the bifurcation occurs is called the bifurcation value of $\mu$. If no bifurcation occurs at an equilibrium $\left(\mathrm{x}^{*}, \mu_{0}\right)$, the equilibrium is said to be hyperbolic.

The bifurcation phenomenon can be related to the notion of structural stability. A dynamical system is called structurally stable if the qualitative dynamical properties of the system persist with small variations in its structure, i.e., when varying the parameters or considering small perturbations of the system. Assume that a dynamical system possesses a unique and asymptotically stable equilibrium then the structural stability implies that the equilibrium is unique and asymptotically stable for different parameter values as well. In other words, a dynamical system is structurally stable if the flow of a slightly varied system is topologically equivalent to the original flow, i.e., if the two trajectories stay close together.

We present briefly, as following, the three types of bifurcation for the onedimensional case, respectively fold, transcritical and pitchfork bifurcations though these bifurcations can occur in higher-dimensional system as well.

\section{Fold Bifurcation}

Consider the differential equation $(A 8.1)$ and let $\left(x^{*}, \mu_{0}\right)=(0,0)$ for simplicity.

Theorem A8.1

(Fold Bifurcation)

Let $f$ in (A8.1) be $C^{2}$ and assume that there is an equilibrium point $\left(x^{*}, \mu_{0}\right)=(0,0)$. If

(1) $[\partial f(0,0) / \partial x]=\lambda=0$,

(2) $\left[\partial^{2} f(0,0) / \partial x^{2}\right] \neq 0$,

(3) $[\partial f(0,0) / \partial \mu] \neq 0$,

i) then, depending on the sign of the expressions in (2) and (3), there are 
ii) no equilibria near $(0,0)$ if $\mu<0(\mu>0)$, and

iii) two equilibria near $(0,0)$ if $\mu>0(\mu<0)$.

The fold bifurcation is sometimes also called a saddle-node bifurcation. Conditions (2) and (3) are called transversality conditions. The prototype equation for the fold bifurcation is $d x / d t=\mu-x^{2}$. If the sign of the transversality conditions are negative and positive, respectively, and the parameter $\mu$ is lower than the bifurcation value $\mu_{0}$ $=0$, no equilibrium exists. For $\mu>\mu_{0}$, two branches of equilibria emerge, one being stable and the other being unstable. Alternatively, if (3) has a negative sign, the bifurcation diagram would appear mirror-imaged with respect to the z-axis. If (2) and (3) are both positive, the stability of the two equilibrium branches is reversed.

Theorem A8.2 (Transcritical Bifurcation)

Let $f$ in (A8.1) be $C^{2}$ and assume that there is an equilibrium point $\left(x^{*}, \mu_{0}\right)=(0,0)$. If

(1) $[\partial f(0,0) / \partial x]=\lambda=0$,

(2) $\left[\partial^{2} f(0,0) / \partial x^{2}\right] \neq 0$,

(3') $\left[\partial^{2} f(0,0) / \partial \mu \partial x\right] \neq 0$,

then, depending on the sign of the expressions in (2) and (3'),

i) the equilibrium $x^{*}$ is stable (unstable) for $\mu<0(\mu>0)$, and

ii) the equilibrium $x^{*}$ becomes unstable (stable) for $\mu>0(\mu<0)$

and a branch of additional stable (unstable) equilibria $x(\mu)$ emerges.

The transcritical bifurcation is thus characterised by an exchange of stability of the origin. The prototype equation for the fold bifurcation is $d x / d t=\mu x-x^{2}$ and the sign of the transversality conditions (2) and (3') are negative and positive, respectively. For $\mu<\mu_{0}=0$ the origin $x=0$ is the only equilibrium point which is stable. If $\mu<$ $\mu_{0}$, the equilibrium $x=0$ becomes unstable and a new equilibrium line $x^{*}(\mu)$ emerges which is stable. Also, if the sign of ( $\left.3^{\prime}\right)$ is reversed, the bifurcation diagram would appear mirror-imaged. If (2) has a positive sign, the stability of the equilibrium for different $\mu$ would be reversed.

As economic examples we mention here only a partial-analytical model of the labour market in case of the fold bifurcation and a "neoclassical" growth model (including the incomplete Inada conditions) in case of the transcritical bifurcation.

A final example of a bifurcation of an equilibrium into two or more stable and unstable equilibria is the so-called pitchfork bifurcation. This bifurcation can occur in dynamical systems of the form (A8.1) with the additional assumption that the function $f$ is an odd function with respect to $x$, i.e., $\left.f(x,)^{\prime}\right)=-f(-x$,$) . When f$ is an odd function, then the sufficient conditions for a transcritical bifurcation are not fulfilled since condition (2) in Theorem A8.1 will be violated for at least one x. Condition (2) will be replaced by the requirement that the third partial derivative with respect to $x$ is different from zero. 


\section{Theorem A8.3}

(Pitchfork Bifurcation)

Let $f$ in (A8.1) be $C^{3}$ and assume that there is an equilibrium point $\left(X^{*}, \mu_{0}\right),=(0,0)$. If

(1) $[\partial f(0,0) / \partial x]=\lambda=0$,

(2') $\left[\partial^{3} f(0,0) / \partial x^{3}\right] \neq 0$,

(3') $\left[\partial^{2} f(0,0) / \partial \mu \partial x\right] \neq 0$,

then, depending on the sign of the expressions in (2') and (3'),

i) the equilibrium $x^{*}$ is stable (unstable) for $\mu<0(\mu>0)$, and

ii) the equilibrium $x^{*}$ becomes unstable (stable) for $\mu>0(\mu<0)$ and two branches of additional stable (unstable) equilibria $x(\mu)$ emerges.

The prototype equation in case of the pitchfork bifurcation is $d x / d t=\mu x-x^{3}$. The sign of the transversality condition (2') and (3') in Theorem A8.3 are negative and positive, respectively, such that a so-called supercritical pitchfork bifurcation occurs with the bifurcating branches representing stable equilibria. As in case of the transcritical bifurcation, the bifurcation diagram in mentioned figure would appear mirror-imaged if the sign of (3') were reversed. If (2') were positive, then the two emerging additional equilibria would be unstable. In that case, a subcritical pitchfork bifurcation would occur.

As application in economics, we mention an abridged version of the Kaldor model with an S-investment function and under the assumption that investment is independent of the capital stock.

All three types of bifurcation presented can occur also in higher-dimensional continuous-time dynamical systems. Then the requirement $\lambda=0$ has to be replaced by the condition that out of the $n$ eigenvalues a single eigenvalue is zero while $k$ eigenvalues are positive and $n-k-1$ eigenvalues are negative. Furthermore, the conditions on the single partial derivatives must be replaced by the appropriate matrix expressions.

There is also the so-called Hopf bifurcation in continuous time, but this requires an at least two-dimensional system. The presentation of the Hopf bifurcation is too large and it is not the topic of this paper. Here, we mention only the prototype equations:

$d x / d t=-y+x\left(\mu-\left(x^{2}+y^{2}\right)\right)$

and

$d y / d t=x+y\left(\mu-\left(x^{2}+y^{2}\right)\right)$

As economic applications we refer only to a transformed version of the same Kaldor model (which serves as a prototype model in nonlinear dynamical economics) and to an augmented IS-LM business cycle model.

In general, under some specific conditions, the presented types of bifurcations occur also in the discrete-time dynamical systems. 

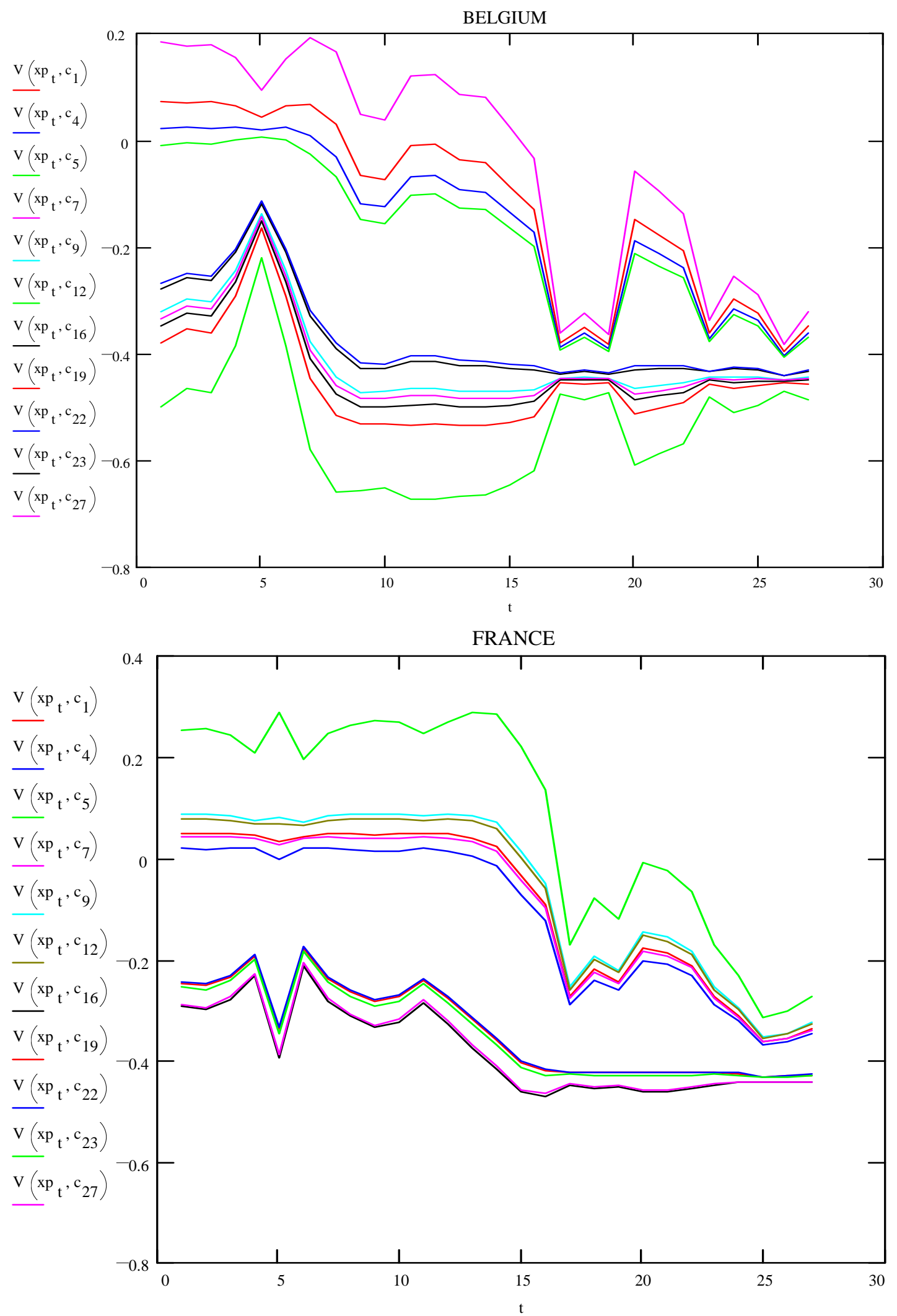
(continued)
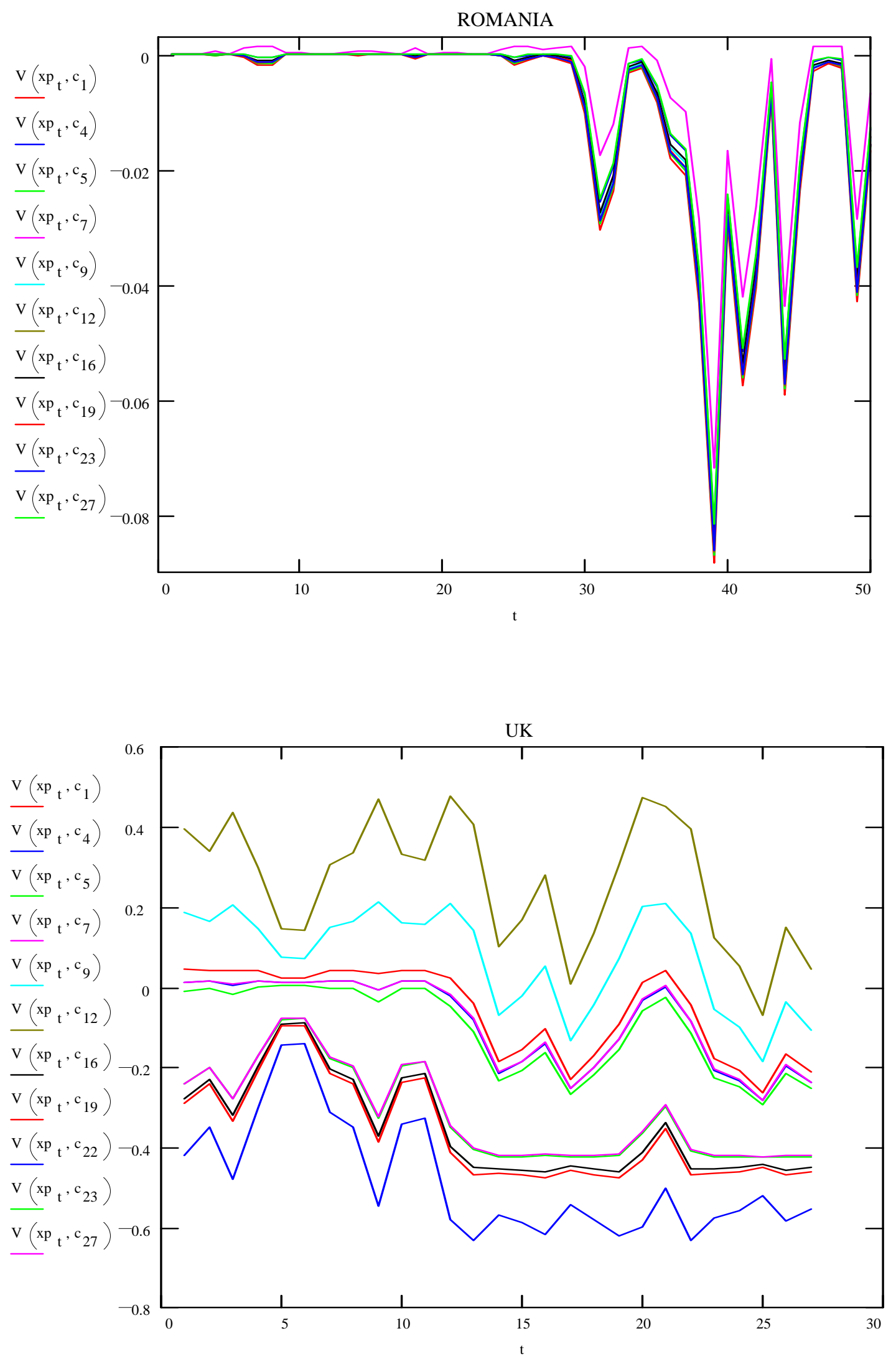